TRANSACTIONS OF THE

AMERICAN MATHEMATICAL SOCIETY

Volume 362, Number 5, May 2010, Pages 2339-2391

S 0002-9947(09)04916-2

Article electronically published on December 3, 2009

\title{
THE CUT-OFF COVERING SPECTRUM
}

\author{
CHRISTINA SORMANI AND GUOFANG WEI
}

\begin{abstract}
We introduce the $R$ cut-off covering spectrum and the cut-off covering spectrum of a metric space or a Riemannian manifold. The spectra measure the sizes of localized holes in the space and are defined using covering spaces called $\delta$ covers and $R$ cut-off $\delta$ covers. They are investigated using $\delta$ homotopies which are homotopies via grids whose squares are mapped into balls of radius $\delta$.

On locally compact spaces, we prove that these new spectra are subsets of the closure of the length spectrum. We prove the $R$ cut-off covering spectrum is almost continuous with respect to the pointed Gromov-Hausdorff convergence of spaces and that the cut-off covering spectrum is also relatively well behaved. This is not true of the covering spectrum defined in our earlier work, which was shown to be well behaved on compact spaces. We close by analyzing these spectra on Riemannian manifolds with lower bounds on their sectional and Ricci curvature and their limit spaces.
\end{abstract}

\section{INTRODUCTION}

Metric spaces and Riemannian manifolds are often studied using Gromov-Hausdorff convergence and Gromov's compactness theorem. However, this convergence, reviewed in Section 5 does not preserve the topology of the space. Thinner and thinner flat tori converge to circles, thus losing a generator of the fundamental group. Sequences of surfaces of higher and higher genus can converge to the Hawaii Ring, a space with an infinitely generated fundamental group and no universal cover [Example 2.4. Sequences of capped cylinders can be seen to converge in the pointed Gromov-Hausdorff sense to cylinders if the base points slide out to infinity [Example 5.3 .

Adding curvature conditions to the spaces in question both restrict their topology and the topology of the limit spaces. Cheeger-Gromoll's Soul Theorem not only demonstrates that complete noncompact spaces with nonnegative sectional curvature have finite topological type, but also that their "holes" are located in a compact soul $\mathrm{ChGr}$. Perelman proved a geometric extension of this result for their limit spaces using work of Sharafutdinov [Sh], Pe1]. More recently Cheeger and Colding have proven a number of results concerning the limits of manifolds with nonnegative Ricci curvature (cf. $\mathrm{Ch}$ and Wei]). The topology of such spaces has been studied extensively by a number of mathematicians (cf. ShSo2]). As one examines this work, it becomes clear that it is not only of importance to understand

Received by the editors October 31, 2007.

2010 Mathematics Subject Classification. Primary 54E45, 53C20.

The first author was partially supported by a grant from the City University of New York PSC-CUNY Research Award Program.

The second author was partially supported by NSF Grant \# DMS-0505733.

(C)2009 American Mathematical Society 2339

Reverts to public domain 28 years from publication 
the topological question concerning the existence of holes in these spaces but also to examine the geometric properties of these holes.

In SoWei3, the authors defined the covering spectrum of a compact length space, $K$. This spectrum measures the size of the one dimensional holes in the space and is closely related to the length spectrum: every element in the covering spectrum is half the length of a closed geodesic,

$$
\text { CovSpec }(K) \subset(1 / 2) \text { Length }(K) .
$$

The covering spectrum is empty when the space is simply connected or is its own universal cover. It is determined using a sequence of covering spaces called $\delta$ covers which unravel curves that do not fit in balls of radius $\delta$. We proved that when compact length spaces $K_{i}$ converge in the Gromov-Hausdorff sense to a compact length space $K$, then their covering spectra converge in the Hausdorff sense:

$$
d_{H}\left(\operatorname{CovSpec}\left(K_{i}\right) \cup\{0\}, \operatorname{CovSpec}(K) \cup\{0\}\right) \rightarrow 0 .
$$

It is possible for elements to converge to 0 as they do on the sequence of thinner and thinner tori, at which point they disappear and are no longer in the covering spectrum. However, elements which converge to a positive value do not disappear in the limit. Furthermore, an element in $\operatorname{CovSpec}(K)$ is the limit of elements in $\operatorname{CovSpec}\left(K_{i}\right)$. In particular, the covering spectrum of the limit space, $K$, of a sequence of simply connected spaces, $K_{i}$, is empty SoWei3.

When studying complete locally compact spaces, it is natural to employ pointed Gromov-Hausdorff convergence. The covering spectrum is not continuous with respect to this convergence. Sequences of manifolds $X_{i}$ with handles sliding out to infinity converge to a space $X$ with no handles, so that we can have $\delta \in$ $\operatorname{CovSpec}\left(X_{i}\right) \forall i \in \mathbb{N}$ yet $\operatorname{CovSpec}(X)=\emptyset$ [Example [5.5. It is even possible for there to be an element in the covering spectrum of the limit space when $\operatorname{CovSpec}\left(X_{i}\right)=\emptyset \forall i \in \mathbb{N}$ [Example 5.3. These difficulties arise because the pointed Gromov-Hausdorff convergence is defined as the Gromov-Hausdorff limit of balls of radius $R$ where the convergence can be slower as we take larger values of $R$.

Further difficulties are caused by the lack of compactness on a single space. Even on a locally compact space the covering spectrum is no longer closely related to the length spectrum on a noncompact space: there can be holes which extend to infinity and decrease in size [Examples 2.19 and 2.20. Those that decrease to 0 are not detected by the covering spectrum, and those that decrease to a constant cause an element in the covering spectrum to exist which is not $1 / 2$ the length of a closed geodesic. This will be explored in a future paper. Here we define a new spectrum which resolves many of these difficulties.

In this paper we introduce the $R$ cut-off covering spectrum and the cut-off covering spectrum to overcome these difficulties. The $R$ cut-off covering spectrum of a pointed space $(X, x)$ detects holes which do not extend outside the closed ball $\bar{B}_{x}(R)$. The cut-off covering spectrum detects holes which do not extend to infinity. A cylinder only has a hole extending to infinity, so its cut-off covering spectrum is empty. We prove that on a complete locally compact length space, $X$, both of these spectra are contained in the closure of the length spectrum because the holes they detect are localized [Theorem 4.18 and Corollary 4.21]. Local compactness is seen to be necessary in Example 4.19.

We prove that the $R$ cut-off covering spectrum is continuous with respect to the pointed Gromov-Hausdorff convergence of the locally compact spaces [Theorem5.7. 
This result is not an immediate extension of our compact results because the $R$ cutoff spectrum is not uniformly localized: it detects any hole which passes into $\bar{B}_{x}(R)$ no matter how far out part of the hole extends. While the elements of the covering spectrum of a compact space are bounded above by the diameter of the space, there is no upper bound on an element in the $R$ cut-off covering spectrum [Example [5.6]. In Example 5.6, one sees a sequence of $X_{i}$ with increasingly large holes such that the hole snaps open to give a simply connected limit $X$. One aspect of our theorem says that if a sequence of spaces $\left(X_{i}, x_{i}\right)$ have elements

$$
\delta_{i} \in \operatorname{CovSpec}_{\text {cut }}^{R}\left(X_{i}, x_{i}\right)
$$

which diverge to infinity, then the holes they detect always snap open in the limit and are no longer holes at all.

Another difficulty in our noncompact setting arises from the fact that the $R$ cutoff covering spectrum is defined using covering spaces, and, as such, homotopies which extend far outside $\bar{B}_{x}(R)$ could influence the value of $\operatorname{CovSpec}_{c u t}^{R}(X, x)$. To handle this issue we develop the concept of the $\delta$ homotopy first introduced in SoWei1. A closed curve is $\delta$ homotopic to a point if it lifts as a closed curve to the $\delta$ cover of the space. We introduce $\delta$ homotopies: maps from rectangular grids to the space which map squares into balls of radius $\delta$ [Lemma 3.4]. This allows us to control the location of the maps, and, in particular, we prove that if a curve is $\delta$ homotopic to a point, then it is $\delta$ homotopic in a bounded region to a collection of possibly trivial loops lying near the boundary of that region [Lemma 3.7]. Later we apply this to localize subsets of the cut-off covering spectrum [Proposition 4.42. We also bound the lengths of curves in a region $A$ with certain $\delta$ homotopic properties in terms of the number of disjoint balls of radius $\delta / 5$ that fit within $A$ [Lemma 3.9. This is useful later for uniformly bounding the size of holes which are detected by the $R$ cut-off covering spectrum in a Gromov-Hausdorff converging sequence of balls.

In Section 2 we review crucial concepts from prior papers, simplifying some and clarifying others. We begin with the classical notions of covering spaces and length spaces and review which metric properties lift from the base space to the cover in Subsection 2.1. Subsections 2.2 and 2.3 review the definitions of delta covers and the covering spectrum as in SoWei1] and SoWei3, respectively. Subsection 2.4 provides a simplified but equivalent definition of the covering spectrum for a complete length space with a universal cover [Definition 2.15] and Theorem 2.17. Note that we do not require the existence of a simply connected cover, but rather just a covering space which covers all other covering spaces in the sense of Spanier $\mathrm{Sp}$.

In Section 3 we develop the theory of $\delta$ homotopy. Similar but distinct concepts appear in the work of Beretovskii-Plaut [BP2] which focuses on the construction of a uniform universal cover which is not a covering space but is simply connected. While the lemmas in Section 3 are very intuitive and have explanatory diagrams, the proofs are necessarily technical and may be skipped by the reader.

In Section 4 we introduce the cut-off covering spectra of pointed metric spaces $(X, x)$. We begin by defining the $R$ cut-off $\delta$ covers, $\tilde{X}_{c u t}^{\delta, R}$, which unravel curves that are not $\delta$ homotopic to loops outside $\bar{B}_{x}(R)$. We prove that when $X$ is a complete locally compact length space, $\tilde{X}_{\text {cut }}^{\delta, R}$ have unique limits as $R$ diverges to infinity and call these limits the cut-off $\delta$ covers [Proposition 4.8. These covers unravel holes which do not extend to infinity. The $R$ cut-off covering spectrum, 
$\operatorname{CovSpec}_{\text {cut }}^{R}(X, x)$, is defined using the $R$ cut-off $\delta$ covers for any metric space while the cut-off covering spectrum $\operatorname{CovSpec}_{\text {cut }}(X)$ is defined using the cut-off $\delta$ covers and is basepoint invariant [Definitions 4.5] and 4.10. By Proposition 4.8, any complete locally compact length space has a well defined cut-off covering spectrum, but we believe algebraic techniques might be used to prove it is well defined for a much larger class of metric spaces.

In Section 4.3 we relate these spectra to the covering spectra and to each other, showing in particular that for any $R_{1}<R_{2}$ and any basepoint $x$ in a metric space $X$ with a well defined cut off covering spectrum, we have

$$
\operatorname{CovSpec}_{\text {cut }}^{R_{1}}(X, x) \subset \operatorname{CovSpec} c_{\text {cut }}^{R_{2}}(X, x) \subset \operatorname{Cov} \operatorname{Spec}_{\text {cut }}(X) \subset \operatorname{CovSpec}(X) .
$$

In Section 4.4, we prove Theorem 4.18, where for a complete locally compact length space, $X$, if $\delta \in \operatorname{CovSpec} \operatorname{Sut}_{\text {cut }}^{R}(X)$, then $2 \delta \in \operatorname{Length}(X)$, which we write as $\operatorname{CovSpec}_{\text {cut }}^{R}(X) \subset(1 / 2) \operatorname{Length}(X)$. As a corollary we then show

$$
\operatorname{CovSpec}_{\text {cut }}(X) \subset C l_{\text {lower }}((1 / 2) \text { Length }(X)),
$$

where $C l_{\text {lower }}(A)$ is the lower semiclosure of the set $A \subset \mathbb{R}$. The lower semiclosure is defined and explored in the appendix, where we prove any spectrum defined in a manner similar to these spectra are lower semiclosed sets [Theorem 7.5]. Example 4.16 demonstrates the necessity of the lower semiclosure in (1.5).

In Section 4.5 we study various topological conditions on metric spaces with well defined cut-off covering spectra. We first recall the loops to infinity property defined in $[\mathrm{So}$ and relate this concept to the emptiness of the cut-off covering spectrum [Theorem 4.23 and Theorem 4.24. Corresponding examples are presented as well. Then we describe the cut-off covering spectrum on product spaces [Theorem 4.26].

In Section 4.6, we introduce a new construction of length spaces which are not locally compact. This construction consists of attaching a "pulled ribbon" to a given space along a line. Example 4.34 demonstrates the necessity of the local compactness condition in Theorem 4.24. Example 4.36 demonstrates that a space with an empty length spectrum can have a nontrivial cut-off covering spectrum demonstrating the necessity of local compactness in Theorem 4.18 ,

In Section 4.7 we localize the $R$ cut-off covering spectrum using the $\delta$ homotopies as mentioned above. Proposition 4.42 shows subsets of the $R$ cut-off covering spectra agree on spaces with isometric balls of sufficient size.

In Section 4.8 we explore

$$
\operatorname{CovSpec}_{\text {cut }}^{R_{2}}(X) \backslash \operatorname{CovSpec} \operatorname{cut}_{\text {cut }}^{R_{1}}(X) \text { when } R_{2}>R_{1} .
$$

In particular Propositions 4.44 and 4.45 together imply that these two spectra are equivalent for $R_{2}$ sufficiently close to $R_{1}$ on locally compact spaces.

In Section [5 we introduce Gromov-Hausdorff convergence, first reviewing the definitions. In Section 5.1 we provide examples demonstrating why the covering spectrum is not continuous with respect to pointed Gromov-Hausdorff convergence: elements can shrink to 0 , disappear in the limit, suddenly appear in the limit, or diverge to infinity.

In Section 5.2 we prove the continuity of the $R$ cut-off covering spectrum [Theorem 5.7 and provide examples clarifying why it is necessary to slightly change $R$ to obtain this continuity. The proof requires two propositions: one controlling 
the fundamental groups of the $R$ cut-off $\delta$ covers and the other proving the $R$ cutoff $\delta$ covers converge. It also strongly relies on the results on $\delta$ homotopies and localization proven in the earlier sections.

In Section 5.3 we prove Theorem 5.12 which states that

$$
\text { for any } \delta \in \operatorname{CovSpec}_{\text {cut }}(X) \text {, there is } \delta_{i} \in \operatorname{CovSpec}_{\text {cut }}\left(X_{i}\right)
$$

such that $\delta_{i} \rightarrow \delta$. In particular if $X_{i}$ are simply connected locally compact spaces that converge to a locally compact space $X$ in the pointed Gromov-Hausdorff sense, then $\operatorname{CovSpec}_{\text {cut }}(X)=\emptyset$ [Corollary [5.13]. This limit space need not be simply connected, as can be seen in Example 5.3.

Further directions of study are suggested in Question 5.14 and Remark 5.15,

In Section 5.4 we prove the pointed Gromov-Hausdorff limits of simply connected spaces either have the loops to infinity property or two ends [Theorem 5.16. In Section 5.5 we investigate the cut-off covering spectra of tangent cones at infinity, proving in Theorem 5.22 that spaces with bounded cut-off covering spectra have tangent cones at infinity with empty covering spectra.

We close the paper with Section [6 on applications to spaces with curvature bounds. Section 6.1 discusses manifolds with nonnegative sectional curvature and consequences of the Cheeger-Gromoll Soul Theorem and work of Sharafutdinov and Perelman. Theorem 6.1 applies to length spaces with curvature bounded below as well and states that if $S$ is the soul of the manifold, then

$$
\operatorname{CovSpec}(S)=\operatorname{CovSpec}\left(T_{R}(S)\right)=\operatorname{CovSpec}(M),
$$

where $T_{R}(S)$ is the tubular neighborhood of the soul.

In Section 6.2 we apply our convergence results to obtain an almost soul theorem [Theorem 6.3] which says that locally (1.8) is approximately true. Corollary 6.5 descibes the local behavior of the covering spectrum of a manifold with sect $\geq-1$. We describe such spaces as having many "subscaled souls".

In Section 6.3 we turn to complete manifolds with nonnegative Ricci curvature. Theorem 6.6] states that the cut-off covering spectrum of such a space is empty unless its universal cover splits isometrically. In particular a manifold with positive Ricci curvature has an empty cut-off covering spectrum [Corollary 6.7.

In Section 6.4 we prove Theorem 6.8 which concerns limits of spaces with lower bounds on their Ricci curvature approaching 0 . We then suggest some open problems related to Ricci curvature and the cut-off covering spectrum and possible local almost soul theorems similar to Corollary 6.5. Conjecture 6.9 suggests an extension of a theorem of the first author from [So] which was used to prove Theorem 6.6. In Conjecture 6.10 we suggest that Theorem 6.8 might then be strengthened to Theorem [6.6] In Conjecture 6.11 we suggest a possible subscaled soul theorem for manifolds with Ricci $\geq-1$ similar to Corollary 6.5. We close by applying Theorem 5.7 and Theorem 6.3 to prove Conjecture 6.10 implies Conjecture 6.11 [Theorem 6.12.

Appendix A provides the background on a concept we call semiclosure, which is needed to describe some properties of the covering spectrum.

In Appendix B we prove Lemmas 8.2 and 8.1 which correctly restate and circumvent Lemma 5.8 of [SoWei3]. While the original Lemma 5.8 was applied to prove Theorem 5.7 of [SoWei3], that the marked length spectrum of a compact length space with a universal determines the covering spectrum, these lemmas apply to complete length spaces which are not compact and do not have universal covers. 
They are used throughout this paper. The appendix closes with an explanation as to why Theorem 5.7 of SoWei3] is correct.

We would like to thank Carolyn Gordon, David Fisher and Ruth Gornet for encouraging us to pursue a further investigation of the covering spectrum. We would like to thank Conrad Plaut for his incisive questions regarding the existence of rectifiable curves that led to Appendix B. Finally we would like to thank Jay Wilkins and the referee for their close reading of the preprint. The first author is grateful to the Courant Institute for its hospitality in Spring 2007.

\section{BACKGROUND}

In Section 2.1 we review the classical notions of covering spaces and length spaces and how the metric structures lift from the base space to the covering space. In Sections 2.2 and 2.3 we review the definitions of Spanier covers, delta covers and the covering spectrum for complete length spaces (geodesic spaces). In Section 2.4 we provide a simplified yet equivalent definition of the covering spectrum of a space when the space has a universal cover [Definition 2.15] and Theorem 2.17. Elements required for the proof of Theorem 2.17 appear in Appendix B.

2.1. Covering spaces and length spaces. This section reviews classical theory of covering spaces and length spaces. First we recall some basic definitions.

Definition 2.1. A metric space is a length space if the distance between points is the infimum of the lengths of curves running between those points. When the infimum is achieved between a given pair of points, we call the minimizing curve a minimal geodesic. When the infimum is achieved for any pair of points, we say the space is a geodesic space.

Given a subset $A$ of a length space $X$, one may either use the restricted metric (which is not a length metric on $A$ ) or the induced length metric which is found by taking the infimum of lengths curves lying in $A$. Note that even when $X$ is a geodesic space, a subset, $A \subset X$, with the induced length metric might not be a geodesic space:

Example 2.2. Let $X$ be the Euclidean plane and

$$
A=\{(x, y): y=|1-x| / j, x \in[-1,1], j \in \mathbb{N}\} \subset X .
$$

Then if $d_{A}$ is the induced length metric, $d_{A}((-1,0),(1,0))=2$ is not achieved.

Recall that a metric space is locally compact iff every point has a precompact neighborhood.

Theorem 2.3 (Hopf-Rinow) (cf. $\mathrm{BBI}, \overline{\mathrm{Gr}}$ ). If $X$ is a complete locally compact length space, then closed balls, $B(x, R)$, in $X$ are compact and $X$ is a geodesic space.

Thus, in particular, complete Riemannian manifolds are geodesic spaces.

Like geodesics in Riemannian manifolds, geodesics in length spaces are defined to be locally minimizing curves. Closed geodesics are geodesics from $S^{1}$ to the space. However, geodesics in length spaces are not necessarily extendable, and they may branch. This can be seen for example in the case of a closed disk (where geodesics end at the boundary) and a tree (where geodesics branch at the vertices). 
Example 2.4. A simple example of a complete length space that we will use repeatedly in this section is a collection of circles of various radii joined at a point, $p$. The distance between points on a single circle is just the shorter arclength between them. Distances between points $q_{1}, q_{2}$ on distinct circles is the sum of the shorter arclength from $q_{1}$ to $p$ and the shorter arclength from $q_{2}$ to $p$. This space is called the Hawaii ring when the collection of radii is $\{1 / j: j \in \mathbb{N}\}$.

The following classical definitions can be found for example in $\mathrm{Sp}$.

Definition 2.5. We say $\bar{X}$ is a covering space of $X$ if there is a continuous map $\pi: \bar{X} \rightarrow X$ such that $\forall x \in X$ there is an open neighborhood $U$ such that $\pi^{-1}(U)$ is a disjoint union of open subsets of $\bar{X}$, each of which is mapped homeomorphically, onto $U$ by $\pi$ (we say $U$ is evenly covered by $\pi$ ).

This is clearly a topological definition which preserves the local topology. Thus if $X$ is locally compact, then any cover, $\bar{X}$, is locally compact as well. Naturally, compactness is a global condition and does not necessarily lift to the cover, as can be seen with the classic example of a line covering a circle. Nevertheless the covering space of a compact space must be locally compact.

Definition 2.6 ([Sp, pp. 62,83]). We say $\tilde{X}$ is a universal cover of $X$ if $\tilde{X}$ is a cover of $X$ such that for any other cover $\bar{X}$ of $X$, there is a commutative triangle formed by a continuous map $f: \tilde{X} \rightarrow \bar{X}$ and the two covering projections.

Note that the Hawaii Ring does not have a universal cover. In [SoWei1, the authors developed a method of detecting whether a compact space like the Hawaii Ring has a universal cover or not.

If $X$ is a length space, then naturally one can define the lengths of curves, $C$, on its covering space, $\bar{X}$, by measuring the lengths of their projections down to $X$ : $L(C):=L(\pi \circ C)$. This defines a metric on $\bar{X}$ which is a length metric, and it is the unique metric on $\bar{X}$ for which $\pi: \bar{X} \rightarrow X$ is a local isometry. Throughout this paper, we will always use this metric on the covering spaces of a length space.

When $X$ is a complete locally compact length space, then $\bar{X}$ is also a complete locally compact length space and, by Hopf-Rinow, it is a geodesic space. However, if $X$ is only a geodesic space, the cover might only be a length space, as can be seen in the following example:

Example 2.7. Let $X$ be a collection of circles of circumference $2 \sqrt{1+(1 / j)^{2}}$ joined at a point. Let $\bar{X}$ be defined as a collection of the sets $A$ defined in Example 2.2 joined to each other in a row:

$$
\bar{X}=\{(x+2 k, y):(x, y) \in A, k \in \mathbb{Z}\} .
$$

Then $\pi: \bar{X} \rightarrow X=\bar{X} / \mathbb{Z}$ is a regular cover and $X$ is a geodesic space, but $\bar{X}$ is only a length space.

Most definitions in this paper are well defined for arbitrary metric spaces. However, on occasion we need a geodesic length structure on the covering spaces or the compactness of closed balls in the covering spaces, and in those settings we require that our spaces be complete locally compact length spaces. We will also employ local compactness when applying the Arzela-Ascoli Theorem or the Gromov Compactness Theorem. 
2.2. Spanier covers and $\delta$-covers. We now introduce a special collection of covers we will call Spanier covers, as they are described in [Sp, Page 81].

Definition 2.8. Let $\mathcal{U}$ be any collection of open sets covering $X$. For any $p \in X$, by $[\mathrm{Sp}$, Page 81$]$ there is a covering space, $\tilde{X}_{\mathcal{U}}$, of $X$ with covering group $\pi_{1}(X, \mathcal{U}, p)$, where $\pi_{1}(X, \mathcal{U}, p)$ is a normal subgroup of $\pi_{1}(X, p)$, generated by homotopy classes of closed paths having a representative of the form $\alpha^{-1} \circ \beta \circ \alpha$, where $\beta$ is a closed path lying in some element of $\mathcal{U}$ and $\alpha$ is a path from $p$ to $\beta(0)$.

It is easy to see that a Spanier cover is a regular or a Galois cover. That is, the lift of any closed loop in $Y$ is either always closed or always open in a Spanier cover. In particular Spanier covers of a collection of circles of various radii will leave some or none of the circles as circles and unravel the other circles completely into a tree.

The following lemma is in Spanier [Sp, Ch.2, Sec.5, 8]:

Lemma 2.9. Let $\mathcal{U}$ and $\mathcal{W}$ both be collections of open sets that cover $X$. Suppose $\mathcal{U}$ refines $\mathcal{W}$ in the sense that for any open set $W$ in $\mathcal{W}$ there is an open set $U \in \mathcal{U}$, such that $U \subset W$. Then the Spanier cover $\tilde{X}_{\mathcal{U}}$ covers $\tilde{X}_{\mathcal{W}}$.

Spanier covers will be used to define various covering spaces in this paper as well as the $\delta$ covers first introduced by the authors in [SoWei1].

Definition 2.10. Given $\delta>0$, the $\delta$-cover, denoted $\tilde{X}^{\delta}$, of a metric space $X$ is defined to be the Spanier cover, $\tilde{X}_{\mathcal{U}_{\delta}}$, where $\mathcal{U}_{\delta}$ is the open covering of $X$ consisting of all open balls of radius $\delta$.

The covering group will be denoted $\pi_{1}(X, \delta, p) \subset \pi_{1}(X, p)$. This is the normal subgroup of $\pi_{1}(X, p)$, generated by homotopy classes of closed paths having a representative of the form $\alpha^{-1} \circ \beta \circ \alpha$, where $\beta$ is a closed path lying in some ball of radius $\delta$ and $\alpha$ is a path from $p$ to $\beta(0)$.

In Example 2.4, the $\delta$ cover of the space consisting of circles of various sizes glued at a common point is a covering space which unravels all the circles of circumference $2 \pi r \geq 2 \delta$ and keeps the smaller circles wrapped as circles. In particular, when $X$ is the figure eight created by joining one circle of circumference $2 \pi$ and one circle of circumference $4 \pi$ at a common point, then $\tilde{X}^{\delta}$ is $X$ itself when $\delta>2 \pi$, it is a real line with circles of circumference $2 \pi$ glued at the points $\{2 j \pi: j \in \mathbb{Z}\}$ when $\delta \in(\pi, 2 \pi]$, and it is the universal cover $\tilde{X}$ when $\delta \leq \pi$.

Note that like all covering spaces, the $\delta$ covers of complete locally compact length spaces are also complete locally compact length spaces and are thus geodesic length spaces. Example 4.36 is a complete geodesic length space with a $\delta$ cover that is not a geodesic length space, so the local compactness condition is required to lift this property even to $\delta$ covers.

The $\delta$ covers of compact spaces are surveyed quickly in the background section of SoWei3. There we proved that $\delta$ covers of metric spaces are monotone in the sense that if $\delta_{1}<\delta_{2}$, then $\tilde{X}^{\delta_{1}}$ covers $\tilde{X}^{\delta_{2}}$, which just follows from Lemma 2.9. See SoWei3, Lemma 2.6].

If one has a space where balls of radius $\delta_{1}$ and $\delta_{2}$ have the same topology, the covering spaces are the same. In fact, for compact spaces we proved the $\delta$ covers are lower semicontinuous in the sense that for any $\delta_{1}>0$ there is a $\delta_{2}<\delta_{1}$ sufficiently close to $\delta_{1}$ such that the two delta covers agree [SoWei3, Lemma 2.7]. This is not true for complete noncompact spaces. In fact, the space of circles of circumference $2 \pi r_{i}$ joined at a point have distinct delta covers for each $\delta_{i}=\pi r_{i}$ so that lower 
semicontinuity fails when there is a sequence $r_{i}$ increasing to $r_{0}$ SoWei3, Example $2.8]$.

2.3. Review of the covering spectrum. In SoWei3] we introduced the covering spectrum on compact metric spaces which is well defined on complete noncompact spaces as well.

Definition 2.11. Given a metric space $X$, the covering spectrum of $X$, denoted $\operatorname{CovSpec}(X)$, is the set of all $\delta>0$ such that

$$
\tilde{X}^{\delta} \neq \tilde{X}^{\delta^{\prime}}
$$

for all $\delta^{\prime}>\delta$.

The covering spectrum of a finite collection of circles of circumference $2 \pi r_{i}$ joined (glued) at a common point is $\left\{\pi r_{i}\right\}$.

For a compact length space the covering spectrum is discrete, and the only accumulation point of the covering spectrum that can occur outside of the covering spectrum is 0 [SoWei3, Prop. 3.2]. This happens for example with the Hawaii Ring, where the circles have circumference $2 \pi r_{j}=2 \pi / j$.

The covering spectra of complete noncompact spaces need not be discrete:

Example 2.12. The covering spectrum of a complete noncompact length space can be $(0, \infty)$, as can be seen by joining the uncountable collection of circles of circumference $2 \pi r$ for every $r \in(0, \infty)$ at a common point. This same covering spectrum can be achieved by taking a joined countable collection of circles of circumference $2 \pi r$ for every $r \in \mathbb{Q}$.

The following lemma is a simple exercise on the definition:

Lemma 2.13. If $\delta_{j} \in \operatorname{CovSpec}(X)$ and $\delta_{j}$ decrease to a positive limit $\delta_{0}>0$, then $\delta_{0} \in \operatorname{CovSpec}(X)$.

Example 2.14. Thus the covering spectrum of the joined collection of circles of circumference $2 \pi r_{j}=2 \pi+2 \pi / j$ is $\{\pi(1+1 / j): j \in \mathbb{N}\} \cup\{\pi\}$. In contrast the covering spectrum of the joined collection of circles of circumference $2 \pi r_{j}=$ $2 \pi-2 \pi / j$ is just $\{\pi(1-1 / j): j \in \mathbb{N}\}$.

This is just an indication of the complexity one encounters when studying the covering spectra of complete noncompact spaces. In the next section we explore this situation, and in subsequent sections we introduce alternative spectra which detect properties that the covering spectrum cannot detect on a complete noncompact space. Further review of the covering spectra of compact spaces will appear below.

2.4. The covering spectrum and deck transforms. In our prior papers, we did not like to assume the space had a universal cover, in part because we were applying $\delta$-covers and the covering spectrum to prove the existence of universal covers. However, if one does assume the existence of a universal cover, then there is a fairly beautiful new perspective on the meaning of the covering spectrum using its relationship with the group of deck transforms $\pi_{1}(X)$ on the universal cover, $\tilde{X}$. When the universal cover is simply connected, this group of deck transforms is isometric to the fundamental group $\pi_{1}(X, p)$.

Recall that a $\delta$ cover, $\tilde{X}^{\delta}$, is defined using a covering group $\pi_{1}(X, \delta, p)$, so with a universal cover we have

$$
\tilde{X}^{\delta}=\tilde{X} / \pi_{1}(X, \delta)
$$


where $\pi_{1}(X, \delta) \subset \pi_{1}(X)$. This provides us with an equivalent definition for the covering spectrum:

Definition 2.15. Given a metric space $X$ with a universal cover, $\tilde{X}$, the covering spectrum of $X$ is the set of all $\delta>0$ such that

$$
\pi_{1}(X, \delta) \neq \pi_{1}\left(X, \delta^{\prime}\right) \quad \forall \delta^{\prime}>\delta
$$

when viewed as subsets of $\pi_{1}(X)$.

We can now use the existence of the universal cover to simplify the definition of $\pi_{1}(X, \delta)$ so that Definition 2.15 can be used to quickly recover the covering spectrum of a space whose deck transforms are well understood. We begin with the following standard definition.

Definition 2.16. Given a complete locally compact length space $X$ with universal cover $\tilde{X}$, for each element $g \in \pi_{1}(X)$ its length $L(g)$ is

$$
L(g)=\inf _{\tilde{x} \in \tilde{M}} d(\tilde{x}, g \tilde{x}) .
$$

Theorem 2.17. Given a complete locally compact length space $X$ with a universal cover, the $\delta$ covering group $\pi_{1}(X, \delta)$ is the subgroup of $\pi_{1}(X)$ generated by deck transforms $g$ with $L(g)<2 \delta$.

To prove this theorem we will apply Lemma 8.1 which states that when $C$ is a rectifiable loop lying in a ball of radius $\delta$, then $C$ is freely homotopic to a product of curves of length $<2 \delta$. This slight restatement of Lemma 5.8 of [SoWei3] is located in Appendix B.

We also need the following lemma regarding the rectifiability of loops representing elements of $\pi_{1}(X, \delta)$ for spaces with universal covers. It is important to note that in the Hawaii Ring, which does not have a universal cover, there are elements of $\pi_{1}(X, \delta)$ which are not rectifiable.

Lemma 2.18. Given a complete locally compact length space $Y$ with a universal cover, if $g \in \pi_{1}(X, \delta)$ has positive length, then it is generated by elements of $\pi_{1}(X, \delta)$ whose representatives have the form $\alpha^{-1} \circ \beta \circ \alpha$ where $\beta$ is rectifiable and lies in balls of radius $\delta$.

It is important to note that we do not claim that the fundamental group always has a rectifiable representative. Recall that spaces exist which do not have simply connected universal covers $\mathrm{Sp}$. These spaces can have elements in their fundamental group which do not have rectifiable curves representing them, but we show that their images in the deck transform group will have rectifiable representative curves.

Proof of Lemma 2.18. Suppose that $L(g)=L_{0}>0$. So for all $x \in \tilde{X}, d(g x, x) \geq$ $L_{0}$. We know $g$ is a product of elements with representatives of the form $\alpha^{-1} \circ \beta^{\prime} \circ \alpha$, where the $\beta^{\prime}$ might not be rectifiable but is contained in balls of radius $<\delta$.

We need only replace each $\beta^{\prime}$ with a rectifiable curve $\beta$ that has the same end points when lifted to the universal cover and which also fits in the $\delta$ ball.

Note that since $[0,1]$ is compact, the image of $\beta^{\prime}$ is a compact set. So there exists an $\epsilon>0$ sufficiently small such that for any $t \in S^{1}, B_{\beta^{\prime}(t)}(5 \epsilon) \subset B_{q}(\delta)$.

Now we lift $\beta^{\prime}$ to the universal cover $\tilde{X}$ and partition the lift $\tilde{\beta}^{\prime}$ into $0=t_{0}<$ $t_{1}<t_{2}<\cdots<t_{N}=1$ so that each segment $\tilde{\beta}^{\prime}\left(\left[t_{i}, t_{i+1}\right]\right)$ lies in a ball of radius $\epsilon$. 
We create a piecewise geodesic, $\tilde{\beta}$, in the universal cover which joins these endpoints with minimal geodesics of length $<2 \epsilon$.

Projecting $\tilde{\beta}$ down to a curve $\beta$, we see $\beta$ has the same endpoints at $\beta^{\prime}$, passes through $\beta^{\prime}\left(t_{i}\right)$ and must also lie in

$$
\bigcup_{i=0}^{N} B_{\beta^{\prime}\left(t_{i}\right)}(2 \epsilon) \subset B_{q}(\delta) .
$$

We now easily prove the theorem:

Proof of Theorem 2.17. Suppose $L(g)<2 \delta$. Then $g$ has a representative loop $\beta$ whose lift runs between some $x$ and $g x$ of length less than $2 \delta$. We do not need to assume compactness of $X$ because of the strict inequality here. This implies $\beta$ has length $<2 \delta$ and thus fits in some ball of radius $\delta$. So $g \in \pi_{1}(X, \delta)$.

Suppose on the other hand $g \in \pi_{1}(X, \delta)$. By Lemma 2.18, either $L(g)=0<2 \delta$ or it is generated by elements of $\pi_{1}(X, \delta)$ with representative loops of the form $\alpha^{-1} \circ \beta \circ \alpha$, where $\beta$ is rectifiable and lies in a ball of radius $\delta$. We just apply Lemma 8.1 to state that $\beta$ is homotopic to a product of loops of length $<2 \delta$. Thus $g$ is generated by elements with representative loops of length $<2 \delta$. Such elements must then have length $<2 \delta$.

On compact length spaces Theorem 2.17 can be combined with the ArzelaAscoli Theorem and Lemma 3.2 to prove CovSpec $(M) \subset(1 / 2) \operatorname{Length}(M)$, where Length $(M)$ is the collection of lengths of closed geodesics $\gamma: S^{1} \rightarrow M$ [SoWei3]. This is not true on complete locally compact length spaces, as the infimum in (2.6) need not be achieved:

Example 2.19. Let $M^{2}$ be the warped product manifold $\mathbb{R} \times_{f(r)} S^{1}$, where

$$
f(r)=2 \operatorname{Arctan}(-r)+2 \pi \text {. }
$$

Here $\pi_{1}(M)$ is generated by a single element $g$ whose length

$$
L(g)=\inf _{r \in(-\infty, \infty)} f(r)=\pi,
$$

but there is no closed curve homotopic to a representative of $g$ whose length is $\pi$.

On a compact Riemannian manifold, $\operatorname{CovSpec}(M)=\emptyset$ implies $M$ is simply connected [SoWei3]. Yet this is not true for complete manifolds:

Example 2.20. Let $M^{2}$ be the warped product manifold $\mathbb{R} \times_{f(r)} S^{1}$, where

$$
f(r)=2 \operatorname{Arctan}(-r)+\pi \text {. }
$$

Given any $\delta>0$, eventually $f(r)<2 \delta$, so any $g \in \pi_{1}(M)$ is represented by a loop of length $<2 \delta$. Thus by Theorem 2.17 the covering spectrum is empty.

Further implications of this perspective on the covering spectrum will be investigated in future joint work. In that paper we will also investigate the slipping group:

Definition 2.21. The slipping group of $X$, denoted $\pi_{\text {slip }}(X)$, is generated by the elements $g \in \pi_{1}(X)$ such that $L(g)=0$. 


\section{Delta homotopies}

In this section we develop the concept of the delta homotopy which we first defined in [SoWei1]:

Definition 3.1. Two loops $\gamma_{1}, \gamma_{2}$ in a metric space, $X$, are called $\delta$-homotopic if $\pi_{\delta}\left(\left[\gamma_{1}\right]\right)=\pi_{\delta}\left(\left[\gamma_{2}\right]\right)$, where $\pi_{\delta}: \pi(X) \rightarrow \pi(X) / \pi(X, \delta)$. In particular $\gamma_{1}$ is $\delta$ homotopic to a point if

$$
\left[\gamma_{1}\right] \in \pi(X, \delta),
$$

which means $\gamma_{1}$ lifts as a closed loop to $\tilde{X}^{\delta}$.

This concept can be used to produce closed geodesics in length spaces:

Lemma 3.2. If $X$ is a length space and $\gamma: S^{1} \rightarrow X$ has $L(\gamma) \leq 2 \delta$ but is not $\delta$ homotopic to a point, then $\gamma$ is a closed geodesic which is minimizing over any interval of half its length and has length $2 \delta$.

This lemma will be applied later when we prove our new spectra are in the length spectrum.

Proof. Since $\gamma$ lifts as a closed loop to the length space $\tilde{X}^{\delta}$, it does not fit in a ball of radius $\delta$. In particular, for any $t \in S_{\delta / \pi}^{1}$ we have

$$
\operatorname{Im}(\gamma) \cap\left(X \backslash B_{\gamma(t)}(\delta)\right) \neq \emptyset
$$

However $L(\gamma)=2 \delta$, so the only point in (3.2) must be $\gamma(t+\delta)$ and $d(\gamma(t+\delta), \gamma(t))$ must be $\delta$. Thus $\gamma$ is minimizing on any subinterval of length $\delta$ including an interval centered at $t=0$.

The remainder of this section will be dedicated to providing a more geometric understanding of $\delta$ homotopies on metric spaces. We will first relate $\delta$ homotopies to grids [Section 3.1] and describe how to localize $\delta$ homotopies [Section 3.2]. Finally we prove a few properties of $\delta$ homotopies that are localized in precompact sets in length spaces [Section 3.3].

While we apply the results in this section to study the cut-off covering spectrum, we prove them first because they apply in a much more general setting and should prove useful for those interested in other concepts. Those who are more interested in the cut-off covering spectrum may jump to Section 4 and only return to this section before continuing to Section [5] on Gromov-Hausdorff convergence. Alternatively one might skim through this section reading only the statements and viewing the accompanying diagrams.

3.1. Using grids to understand $\delta$ homotopies. Before we can transform our original, somewhat algebraic, definition of $\delta$ homotopy [Definition 3.1 into a geometric statement about grids on metric spaces, we need to examine the definition closely. Clearly it is base point independent. So if a curve $C$ is $\delta$ homotopic to a point, then $\alpha C \alpha^{-1}$ is also $\delta$ homotopic to a point. So it is often easier to think of $\gamma_{1}$ as $\delta$ homotopic to $\gamma_{2}$ if we join them to a common point via curves $\alpha_{1}$ and $\alpha_{2}$ and then say $\alpha_{1} \gamma_{1} \alpha_{1}^{-1}$ is $\delta$ homotopic to $\alpha_{2} \gamma_{2} \alpha_{2}^{-1}$, which is the same as saying

$$
\alpha_{1} \gamma_{1} \alpha_{1}^{-1}\left(\alpha_{2} \gamma_{2} \alpha_{2}^{-1}\right)^{-1}=\alpha_{1} \gamma_{1} \alpha_{1}^{-1} \alpha_{2}^{-1} \gamma_{2}^{-1} \alpha_{2}
$$

is $\delta$ homotopic to a point. In this sense we make the following definition. 
Definition 3.3. A collection of loops $\gamma_{1}, \gamma_{2}, \ldots, \gamma_{k}$ is $\delta$ homotopic to a point if there exist curves $\alpha_{i}$ mapping a base point $p$ to $\gamma_{i}(0)$ and such that

$$
\alpha_{1} \gamma_{1} \alpha_{1}^{-1} \alpha_{2} \gamma_{2} \alpha_{2}^{-1} \cdots \alpha_{k} \gamma_{k} \alpha_{k}^{-1}
$$

is $\delta$ homotopic to a point.

The ordering of the loops is important in this definition. If $\gamma_{1}, \gamma_{2}$ is $\delta$ homotopic to a point, then $\gamma_{1}$ is $\delta$ homotopic to $\gamma_{2}^{-1}$.

Lemma 3.4. A loop $C$ of length $L$ in a metric space is $\delta$ homotopic to a point iff there is a $\delta$ homotopy $H: G \rightarrow X$, where $G$ is an $N \times M$ grid of unit squares such that $H(0, y)=C(y L / M), H(x, 0)=H(x, M)=H(N, y)=C(0)$ and such that the image under $H$ of each square in the grid is contained in a ball of radius $\delta$.
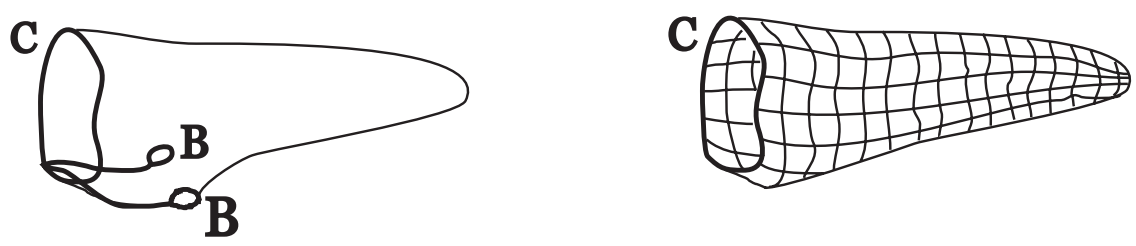

FiguRE 1

In some sense this lemma is intuitively obvious. See Figure 1. Special cases of this lemma were used within some of the proofs in SoWei1. Writing out the proof is a bit technical, and so first we set some notation. Let $\beta_{j, k}$ be the image of the clockwise loop around the square $(j, k),(j, k+1),(j+1, k+1),(j+1, k)$. Let $\alpha_{j, k}$ be the image of the line segment from $(j, 0)$ to $(j, k)$. Let $\bar{\alpha}_{j}$ be the image of the line segment from $(j, 0)$ to $(j-1,0)$.

Proof. If such a homotopy exists, then define $C_{j}(t)$ to be the loop $H(j, t)$ from $t$ to $M$ so $C_{0}(t)=C(t L / M)$ and $C_{N}(t)$ is a point. Note that $C_{0}$ is just $C$. Furthermore each

$\alpha_{j, 0} \beta_{j, 0} \alpha_{j, 0}^{-1} \alpha_{j, 1} \beta_{j, 1} \alpha_{j, 1}^{-1} \cdots \alpha_{j, M} \beta_{j, M} \alpha_{j, M}^{-1}$ is homotopic to $C_{j}(t)\left(\bar{\alpha}_{j} C_{j-1}(t) \bar{\alpha}_{j}^{-1}\right)^{-1}$

within the image of the grid. Thus, by the definition of the $\delta$ cover,

$$
C_{j}(t)\left(\bar{\alpha}_{j} C_{j-1}(t) \bar{\alpha}_{j}^{-1}\right)^{-1}
$$

lifts as a closed loop to the $\tilde{X}^{\delta}$, and so $C_{j}$ and $C_{j-1}$ are $\delta$ homotopic to each other. Thus $C$ is $\delta$ homotopic to $C_{N}$ which is a point.

Conversely, if $C$ is $\delta$ homotopic to a point, then by the definition of the $\delta$ cover, $C$ is homotopic to a collection of curves $\alpha_{i} \beta_{i} \alpha_{i}^{-1}$, where $\beta_{i}$ are in balls of radius $\delta$. So we take the homotopy $\bar{H}:[0, N] \times[0, M] \rightarrow X$ so that $\bar{H}(0, t)=C(t M / L)$, $\bar{H}(s, 0)=\bar{H}(s, 1)=C(0)$ and

$$
\bar{H}(N, t)=\alpha_{1} \beta_{1} \alpha_{1}^{-1} \alpha_{2} \beta_{2} \alpha_{2}^{-1} \cdots \alpha_{k} \beta_{k} \alpha_{k}^{-1}(t) .
$$

Using the uniform continuity of the homotopy $\bar{H}$ we can choose $N$ and $M$ large enough that each square in the grid is within a ball of radius $\delta$. We can also insure, possibly by adding a few more columns to allow for a slow homotopy between reparametrizations, that each $\beta_{j}$ starts at a $t_{j}$ and ends at a $t_{j}+1$ where $t_{j}$ are integers. 
We now add a gridded column of unit squares on the right side of the homotopy. The horizontal bars will have constant images. The verticals will agree with $\bar{H}(N, t)$ whenever this is part of an $\alpha$ curve but will take the value $\bar{H}\left(N, t_{j}\right)$ for $t \in\left[t_{j}, t_{j}+1\right]$. In this way most of the new squares will be in subsegments of the $\alpha$ curves, and the selected new squares at the $t_{j}$ points will have images equal to $\beta_{j}$ and thus lie in balls of radius $\delta$.

Finally we add more columns to allow for a homotopy from the curve

$$
\alpha_{1} \alpha_{1}^{-1} \alpha_{2} \alpha_{2}^{-1} \cdots \alpha_{k} \alpha_{k}^{-1}
$$

to a point. This can be done just by contracting along each $\alpha_{j}$. In this way we complete the homotopy. Then we restrict the homotopy to the grid points, and we are finished.

Lemma 3.5. If $H$ is a $\delta$ homotopy to a metric space, then there exists $\epsilon \in(0, \delta)$ sufficiently close to $\delta$ that $H$ is an $\epsilon$ homotopy.

In fact on compact spaces, one then has $\tilde{X}^{\epsilon}=\tilde{X}^{\delta}$ as proven in Lemma 2.7 of SoWei3.

Proof. By Definition 3.6. every square $S_{i, j}$ in the domain of $H$ is mapped into a ball $B_{q_{i, j}}(\delta)$. Since $H\left(S_{i, j}\right)$ is a closed set, lying in an open ball, it fits in a smaller open ball $B_{q_{i, j}}\left(\delta_{i, j}\right)$ with $\delta_{i, j}<\delta$. Let

$$
\epsilon=\max \left\{\delta_{i, j}: i=1, \ldots, N, j=1, \ldots, M\right\} .
$$

3.2. $\delta$ homotopies in subsets. The following extension of the definition of $\delta$ homotopy takes full advantage of Lemma 3.4. Note that this extension only requires $X$ to be a metric space.

Definition 3.6. A loop $C$ of length $L$ in a metric space, $X$, is $\delta$ homotopic in $A \subset X$ to a point if there is a $\delta$ homotopy $H: G \rightarrow A$, where $G$ is an $N \times M$ grid of unit squares such that $H(0, y)=C(y L / M), H(x, 0)=H(x, M)=H(N, y)=C(0)$ and such that the image under $H$ of each square in the grid is contained in a ball of radius $\delta$.

We say a curve $C_{0}$ is $\delta$ homotopic in $A$ to a collection of loops $C_{1}, C_{2}, \ldots, C_{k}$ if there exist paths $\alpha_{j}$ from $C_{0}(0)$ to $C_{j}(0)$ lying in $A$ such that

$$
C_{0}^{-1} \alpha_{1} C_{1} \alpha_{1}^{-1} \alpha_{2} C_{2} \alpha_{2}^{-1} \cdots \alpha_{k} C_{k} \alpha_{k}^{-1}
$$

is $\delta$ homotopic in $A$ to a point. We will say $C_{0}$ is $\delta$ homotopic in $A$ to a collection of loops in $B \subset A$ if the loops $C_{j}$ lie in $B$ but we do not require the paths $\alpha_{j}$ to lie in $B$. Similarly one can define $\delta$ homotopies in $A$ between two collections of curves.

Suppose we have a curve which is $\delta$ homotopic in a set $A$ to a point and we would like to restrict the $\delta$ homotopy to a set $B \subset A$. Parts of the $\delta$ homotopy may well leave $B$, and so they need to be chopped off. This provides new curves where the homotopy is chopped. See Figure 4 for a glimpse of an application.

Lemma 3.7. Given a $\delta$ homotopy in $A, H: G \rightarrow A$ from a curve $\gamma$ to a point, and given a set $B$ contained in $A$ such that $\gamma \subset B$, then $\gamma$ is $\delta$ homotopic in $B$ to a collection of curves $\gamma_{1}, \gamma_{2}, \ldots, \gamma_{k}$ such that each $\gamma_{j}$ lies in $B$ and the tubular neighborhood $T_{2 \delta}(A \backslash B)$. 

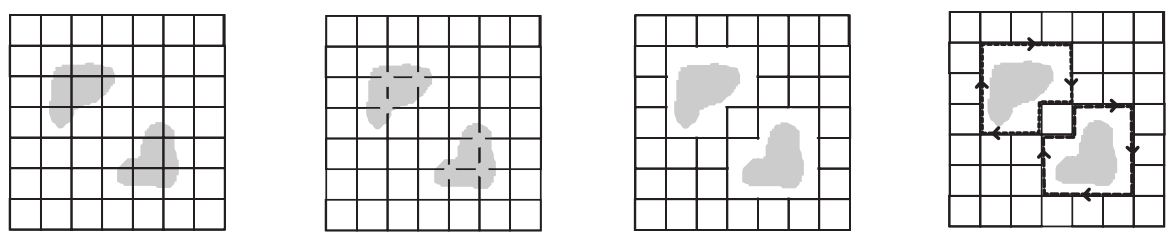

FiguRe 2

Figure 2 depicts this lemma and the idea of the proof. The grey regions are the pullback of the $A \backslash B$ to the grid of the initial homotopy. In the figure the collection is just a pair of curves. Once we have the final picture in the figure, we can apply Lemma 3.8 stated and proven below to justify that images of the pair of curves produced in the last step of the picture are indeed $\delta$ homotopic to the initial curve.

Lemma 3.8. Given a $\delta$ homotopy in $A, H: G \rightarrow A$, from a curve $\gamma$ to a point, and a subset of squares $G^{\prime} \subset G$ such that the image of $C l\left(G \backslash G^{\prime}\right)$ is contained in a set $B \subset A$. Here by closure, we are including the boundary of $G^{\prime}$. Suppose $G^{\prime}$ has connected components $G_{1}, \ldots, G_{k}$. Let $\gamma_{j}$ be the boundary of $G_{j}$ running around clockwise so that the image of $\gamma_{j}$ lies in $B$.

Then $\gamma$ is $\delta$ homotopic in $B$ to the collection of curves $\alpha_{j} \gamma_{j} \alpha_{j}^{-1}$ where $\alpha_{j}$ are paths lying in $B$ or, equivalently, is freely $\delta$ homotopic in $B$ to the collection of curves $\gamma_{j}$.

Intuitively this can be seen because there are only squares that fit in balls of radius $\delta$ running between them. You might wish to skip the proof if you intuitively believe the process. For the intuitive idea see Figure 3 ,

Proof. We now rigorously construct a sequence of collections of curves so that each collection is $\delta$ homotopic to the next. We begin with $\gamma_{0}$ which is the image of the boundary of the entire grid $G_{0}=G$. Each $G_{i}$ will be a subset of $G_{i-1}$ created by removing one square, and at each step our collection of curves will be the boundary of $G_{i}$. We know that we can create a sequence of $G_{i}$ so that we eventually arrive at $G_{I}=G^{\prime}$. We just need to verify that we have a $\delta$ homotopy running from each boundary to the next. There are three cases.

The first case we encounter occurs when removing a square does not change the number of connected components of the subgrid. This is seen in the first part of Figure 3. A square is removed from the side on one region. We need to show that a curve of the form $\mathrm{C}_{1} \mathrm{H}_{2} \mathrm{H}_{3}$ is $\delta$ homotopic to $\mathrm{C}_{1} \mathrm{H}_{1}^{-1} \mathrm{H}_{4}^{-1}$ when $H_{1}, H_{2}, H_{3} H_{4}$ is a loop in a ball of radius $\delta$ because it is the image of a single square. To construct the $\delta$ homotopy, we set $H(0, t)$ to be the required

$$
\mathrm{C}_{1} \mathrm{H}_{2} \mathrm{H}_{3}\left(\mathrm{C}_{1} \mathrm{H}_{1}^{-1} \mathrm{H}_{4}^{-1}\right)^{-1}=\mathrm{C}_{1} \mathrm{H}_{2} \mathrm{H}_{3} \mathrm{H}_{4} \mathrm{H}_{1} \mathrm{C}_{1}^{-1} \text {. }
$$

This time we put all of $H_{2} H_{3} H_{4} H_{1}$ into one integer segment and stretch the $C_{1}$ enough that each segment lies in a $\delta$ ball. We add the second column to the grid keeping everything as in the first column except for the $H_{2} H_{3} H_{4} H_{1}$ segment, which is now just set to $H_{2}(0)=C_{1}(L)$. Thus the image of the grid thus far is contained in the images of the old curves which are in $B$, and all the squares are in $\delta$ balls trivially. The rest of the homotopy is a classical homotopy contracting $C_{1} C_{1}^{-1}$ to a point, and we take as many columns as necessary so that everything is done slowly enough to fit in balls of radius $\delta$. This portion is contained in $\operatorname{Im}\left(C_{1}\right) \subset B$, so we 

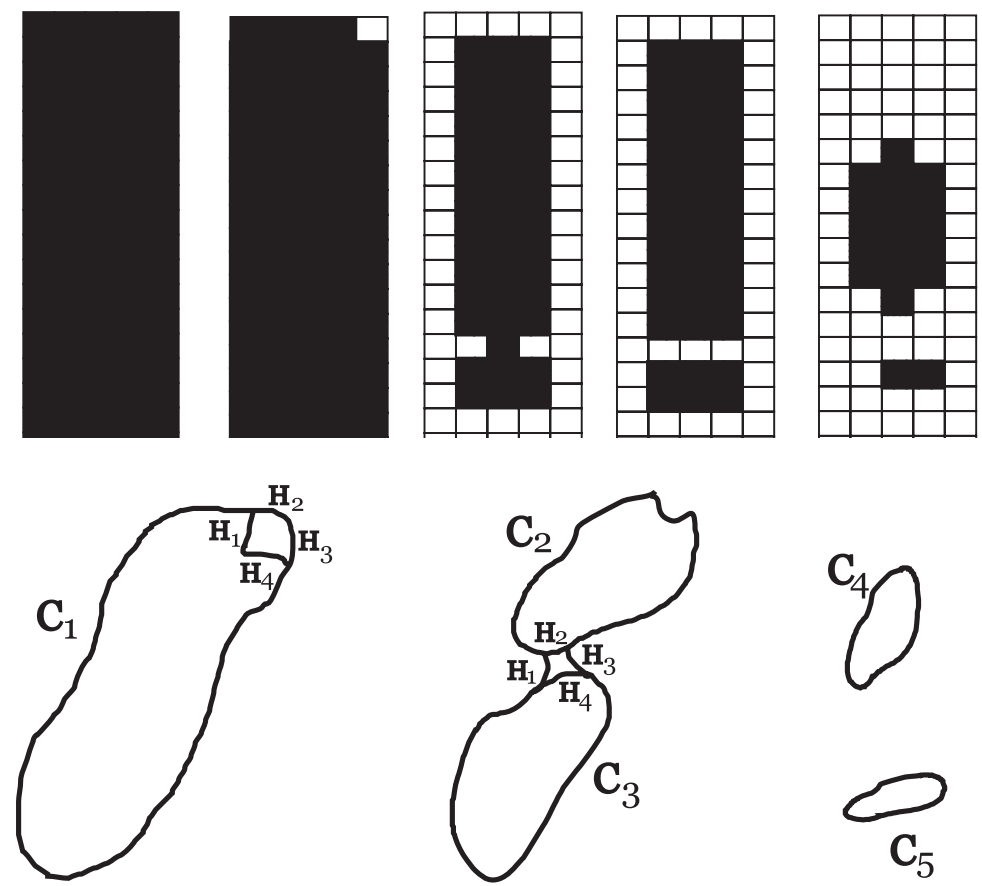

Figure 3. In this figure $\gamma_{0}=C_{1} H_{2} H_{3}$, and we prove it is $\delta$ homotopic in $B$ to the pair of curves $\gamma_{1}=C_{4}$ and $\gamma_{2}=C_{5}$. The grids are drawn above. The first rectangle is filled in completely so we can view our $\gamma_{0}$ as the boundary of the full dark region. The last rectangle has $G^{\prime}$ darkened and its two connected components $G_{1}$ above $G_{2}$. Intuitively we say that the two inner curves should be $\delta$ homotopic to the outer curve because of all the squares between them. The rectangles in between show how we can run through a sequence of subsets of the grid creating a $\delta$ homotopy from $\gamma_{0}$ to the pair $\gamma_{1}$ and $\gamma_{2}$.

are done. It is also possible that the square would be attached on only one side, but this is equally easy.

The second possible case, depicted in the center of Figure 3, is when the square which is removed divides a region into two connected components. So we must show that $\mathrm{C}_{3} \mathrm{H}_{1} \mathrm{C}_{2} \mathrm{H}_{3}$ is $\delta$ homotopic in $B$ to the pair of curves $\mathrm{C}_{3} \mathrm{H}_{4}^{-1}$ and $\mathrm{C}_{2} \mathrm{H}_{2}^{-1}$ given that $\mathrm{H}_{1} \mathrm{H}_{2} \mathrm{H}_{3} \mathrm{H}_{4}$ is the image of a square and so lies in a ball of radius $\delta$. Using $H_{4} H_{1}$ to run $C_{2} H_{2}$ to a common base point, we will construct a $\delta$ homotopy in $B$ from

$$
\left(C_{3} H_{1} C_{2} H_{3}\right)\left(\left(H_{4} H_{1}\right) C_{2} H_{2}^{-1}\left(H_{4} H_{1}\right)^{-1}\right)^{-1}\left(C_{3} H_{4}^{-1}\right)^{-1}
$$

to a point. This is already homotopic within its image to

$$
\mathrm{C}_{3} \mathrm{H}_{1} \mathrm{C}_{2} \mathrm{H}_{3}\left(\mathrm{H}_{4} \mathrm{H}_{1}\right) \mathrm{H}_{2} \mathrm{C}_{2}^{-1}\left(\mathrm{H}_{4} \mathrm{H}_{1}\right)^{-1} \mathrm{H}_{4} \mathrm{C}_{3}^{-1},
$$

which is homotopic within its range to

$$
\mathrm{C}_{3} H_{1} \mathrm{C}_{2} H_{3}\left(H_{4} H_{1}\right) H_{2} C_{2}^{-1} H_{1}^{-1} C_{3}^{-1} \text {. }
$$


Once again we set this up as the first column so that each collection of curves $H_{j}$ fits in a single unit segment and the $C_{j}$ are spread out so that they divided into pieces of length less than $\delta$. Our second column will be set up so that all the horizontal bars are constant and the new vertical line is the same as before except that the segment with $H_{3}\left(H_{4} H_{1}\right) H_{2}$ is not just the fixed point $H_{3}(0)=C_{2}\left(L_{2}\right)$. So our new column is

$$
\mathrm{C}_{3} \mathrm{H}_{1} \mathrm{C}_{2} \mathrm{C}_{2}^{-1} \mathrm{H}_{1}^{-1} \mathrm{C}_{3}^{-1}
$$

but this can be contracted via a homotopy lying on its image to a point, so we just provide that homotopy enough columns so that the images of all the squares lie in $\delta$ balls. So we are done. Note that the order of the new collection was important so that this last step would untangle.

In fact there are cases where the square that is removed might separate into three or even four regions. This follows exactly as above that the regions need to be selected in clockwise order around the square to get the last step to untangle.

The third case is the situation where removing a square removes a segment from the collection. That situation is trivial. Anytime a collection of curves includes a loop within a $\delta$ ball it is $\delta$ homotopic to the collection with the ball removed.

Thus we have shown that no matter how we remove the square, we can show that each collection of curves is $\delta$ homotopic to the next collection by carefully replacing one curve by a new curve or a new curve by a collection of new curves in the right order until finally one has the boundary of the given region $G^{\prime}$.

We can now return to the proof of Lemma 3.7, See Figure 2,

Proof of Lemma 3.7, Let $H$ be the given $\delta$ homotopy. Remove all vertices in $G$ which are mapped by $H$ into $A \backslash B$. Remove all the squares touching these vertices. This gives our collection of squares $G^{\prime}$ which satisfies the condition of Lemma 3.8 . So we obtain a collection $\gamma_{1}, \ldots, \gamma_{k}$ which are $\delta$ homotopic in $B$ to $\gamma$, where each $\gamma_{j}$ lies in the boundary of $G^{\prime}$. Thus every point $q$ which lies on a $\gamma_{j}$ is on the image of a square which includes one of the original removed points $z$. So $q$ and $z$ lie in a common ball of radius $\delta$ and $z \in A \backslash B$. Thus $q \in T_{2 \delta}(A \backslash B)$.

3.3. Compactness and $\delta$ homotopies. One very nice attribute of $\delta$ homotopy classes of curves is that they interact well with compactness on length spaces so that one can control the lengths of curves in a given class. See Section 2.1 for a review of these concepts.

Lemma 3.9. Let $\mathcal{C}$ be a set of loops in a precompact length space, $Z$, which includes a trivial loop. Suppose there is a curve $C$ in $Z$ which is not $\delta=5 \rho$ homotopic to any collection of curves in $\mathcal{C}$. Suppose the number of disjoint balls of radius $\rho$ lying in $Z$ is bounded above by a finite number $N$. Then there exists a curve $\gamma$ in $Z$ which is not $\delta=5 \rho$ homotopic to any collection of curves in $\mathcal{C}$ and has length $\leq 5 N \rho$.

Note here that we cannot just take $C$ to be a trivial loop (a point), because then it would be homotopic to the trivial loop in $\mathcal{C}$. In our application $\mathcal{C}$ will be all loops located outside a given set, but this more general statement is equally valid and possibly useful to others. Our space $Z$ will be a subset of a larger space using the induced length metric and thus might not be complete.

Proof. Take a maximal disjoint collection of balls of radius $\rho$ centered at points

$$
Y=\left\{y_{j}: j=1, \ldots, N\right\} \in Z \text {. }
$$


So the tubular neighborhood of radius $2 \rho$ of this finite collection of points contains all of $Z$.

Take $C:[0, L] \rightarrow Z \subset T_{2 \rho}(Y)$ parametrized by an arclength which is not $5 \rho$ homotopic to any collection of curves in $\mathcal{C}$. We will use $C$ to construct a shorter such curve. Define $0=t_{0}<t_{1}<\cdots<t_{k}=1$ such that $t_{j}-t_{j-1}=\rho$ for $j<k$ and $t_{k}-t_{k-1}<\rho$. Define $\sigma:[0, L] \rightarrow Z$ so that $\sigma\left(t_{j}\right)$ is a point in $Y$ closest to $C\left(t_{j}\right)$. Then

$$
d_{A}\left(\sigma\left(t_{j}\right), \sigma\left(t_{j+1}\right)\right) \leq d_{A}\left(\sigma\left(t_{j}\right), C\left(t_{j}\right)\right)+\rho d_{A}\left(C\left(t_{j+1}\right), \sigma\left(t_{j+1}\right)\right)<5 \rho,
$$

and we can join the points in $\sigma$ by curves in $A$ of length $<5 \rho$. We can also join $C\left(t_{j}\right)$ to $\sigma\left(t_{j}\right)$ by a curve $h_{j}$ in $A$ of length $<2 \rho$. Thus we have a collection of squares

$$
h_{j} \sigma\left(\left[t_{j}, t_{j}+1\right]\right) h_{j+1}^{-1} C\left(\left[t_{j}, t_{j+1}\right]\right)^{-1} \subset B_{\sigma\left(t_{j}\right)}(5 \rho) .
$$

So $C$ is $5 \rho$ homotopic to $\sigma$. Thus $\sigma$ is not $5 \rho$ homotopic to any collection of curves in $\mathcal{C}$. If $k \leq N$, then $L(\sigma) \leq 5 k \rho \leq 5 N \rho$, and we are done.

If $k>N$, then by the pigeonhole principle and the fact that $\sigma\left(t_{j}\right) \in Y$ for $j=0 \ldots k$, with $\sigma\left(t_{0}\right)=\sigma\left(t_{k}\right)$, we see that there must exist a pair $m, n \in\{0, \ldots, k-1\}$ with $|m-n| \leq N$ such that $\sigma\left(t_{n}\right)=\sigma\left(t_{m}\right)$. This allows us to break our loops $\sigma$ into two loops, one of which is of length $\leq 5 \rho N$. If the other loop is longer, apply the pigeonhole principle to that loop and break off another loop of length $\leq 5 \rho N$. Repeating this at most finitely many times, we see that our original curve $\sigma$ is really a concatenation of loops all of which have length $\leq 5 \rho N$.

We claim one of these short loops must not be $5 \rho$ homotopic to any collection of curves in $\mathcal{C}$. Otherwise, all the of them are $5 \rho$ homotopic to some collection of curves in $\mathcal{C}$, and so their concatenation must be $5 \rho$ homotopic to a concatenation of that collection.

\section{The Cut-ofF COVERING SPECTRUm}

It is natural when studying complete noncompact manifolds to remove the ends of the manifolds before beginning the analysis. This technique works well for any complete locally compact metric spaces. In fact, it is standard to refer to pointed spaces, $(X, x)$, with a special base point $x \in X$. In this vein of thought, we define the cut-off covering spectra. We begin by defining the $R$ cut-off $\delta$ covers and $R$ cut-off covering spectra, $\operatorname{CovSpec}_{c u t}^{R}(X)$, which are blind to everything outside a fixed ball of radius $R$. Next we define the cut-off $\delta$ covers by taking $R \rightarrow \infty$ and define the cut-off covering spectrum, $\operatorname{CovSpec} \operatorname{cut}_{\text {cut }}(X)$, based on them.

While the covering spectrum is not well related to the length spectrum on length spaces which are only complete and locally compact, as was seen in Example 2.19, we do prove the $\operatorname{CovSpec}_{\text {cut }}^{R}(X) \subset(1 / 2) L(X)$ and $\operatorname{CovSpec}_{\text {cut }}(X) \subset$ $C l_{\text {lower }}((1 / 2) L(X))$ on such spaces. We then review the loops to infinity property and prove such loops are not detected by the cut-off covering spectra. We close the section with two technical subsections: one establishing that the $R$ cut-off covering spectrum is truly localized and the other describing how $\operatorname{Cov} \operatorname{Spec}_{c u t}^{R}(X)$ changes as one varies $R$. These results will be applied to establish the continuity properties of these cut-off spectra in Section 5 . 
4.1. The $R$ cut-off $\delta$ covers and $\operatorname{Cov} \operatorname{Spec}_{c u t}^{R}(X)$. The $R$ cut-off covering spectrum is a basepoint dependent concept. It is defined on pointed metric spaces $(X, x)$ which are metric spaces with given basepoints. We begin with the corresponding covering spaces. Recall Definition 2.8 on the Spanier cover.

Definition 4.1. Given a pointed metric space $(X, x)$, the $R$ cut-off $\delta$ cover based at $x$, denoted $\tilde{X}_{\text {cut }}^{\delta, R}$ or $\tilde{X}_{\text {cut } x}^{\delta, R}$, is the Spanier cover corresponding to the open sets

$$
\left\{B_{p}(\delta): p \in X\right\} \cup\left\{X \backslash \bar{B}_{x}(R)\right\} .
$$

When the basepoint is obvious we will omit it.

Lemma 4.2. The $R$ cut-off $\delta$ cover based at $x$ is covered by the $\delta$ cover. In fact

$$
\tilde{X}_{c u t}^{\delta, R}=\tilde{X}^{\delta} / G(R),
$$

where $G(R)$ is the subgroup of $\pi_{1}$ generated by elements with representative loops of the form $\alpha \circ \beta \circ \alpha^{-1}$ where $\beta \in M \backslash \bar{B}_{x}(R)$.

Proof. By definition $\pi_{1}\left(\tilde{X}_{\text {cut }}^{\delta, R}\right)$ is generated by loops of the form $\alpha \circ \beta \circ \alpha^{-1}$, where $\beta$ is either in a ball of radius $\delta$ or in $M \backslash \bar{B}_{x}(R)$. So it is generated by elements in $\pi_{1}\left(\tilde{X}^{\delta}\right)$ and elements in $G(R)$. Thus

$$
\tilde{X}_{\text {cut }}^{\delta, R}=\tilde{X} / \pi_{1}\left(\tilde{X}_{\text {cut }}^{\delta, R}\right)=\left(\tilde{X} / \pi_{1}\left(\tilde{X}^{\delta}\right)\right) / G(R)=\tilde{X}^{\delta} / G(R) .
$$

Lemma 4.3. If $B_{x_{1}}\left(R_{1}\right) \subset B_{x_{2}}\left(R_{2}\right)$ in a metric space $X$ and $\delta_{1} \leq \delta_{2}$, then $\tilde{X}_{\text {cut }}^{\delta_{1}, R_{1}}$ based at $x_{1}$ covers $\tilde{X}_{\text {cut }}^{\delta_{2}, R_{2}}$ based at $x_{2}$.

Proof. Just apply Lemma 2.9 which is proven in Sp.

Example 4.4. A cylinder is its own $R$ cut-off $\delta$ cover for all $R>0$ and all $\delta>0$.

Definition 4.5. Given a pointed metric space $(X, x)$, the $R$ cut-off $\delta$ spectrum, denoted $\operatorname{CovSpec} R$ cut $(X)$ or $\operatorname{CovSpec}_{\text {cut }}^{R}(X, x)$, is the collection of $\delta>0$ such that

$$
\tilde{X}_{\text {cut }}^{\delta_{1}, R} \neq \tilde{X}_{\text {cut }}^{\delta, R}
$$

for all $\delta_{1}>\delta$.

Note that by Lemma 4.3 and Theorem 7.5. $\operatorname{CovSpec}_{c u t}^{R}(X)$ is a lower semiclosed set for any metric space $X$.

The following lemma was known for compact length spaces in [SoWei3]:

Lemma 4.6. Given a pointed metric space $X$, if $\left[\delta_{1}, \delta_{0}\right) \cap \operatorname{CovSpec}_{\text {cut }}^{R}(X)=\emptyset$, then $\tilde{X}_{\text {cut }}^{\delta_{1}, R}=\tilde{X}_{\text {cut }}^{\delta_{0}, R}$.

Proof. Let

$$
A=\left\{\delta \in\left[\delta_{1}, \delta_{0}\right): \tilde{X}_{\text {cut }}^{\delta, R}=\tilde{X}_{\text {cut }}^{\delta_{1}, R}\right\} \subset\left[\delta_{1}, \delta_{0}\right) .
$$

Claim. $\sup \{A\}=\delta_{0}$. Otherwise $\sup \{A\}=\delta^{\prime}<\delta_{0}$. By assumption, $\delta^{\prime} \notin$ $\operatorname{CovSpec}_{\text {cut }}^{R}(X, x)$. Therefore there is $\delta^{\prime \prime}>\delta^{\prime}$ such that $\tilde{X}_{\text {cut }}^{\delta^{\prime}, R}=\tilde{X}_{c u t}^{\delta^{\prime \prime}, R}$, contradicting the fact that $\delta^{\prime}$ is the supremum.

So there exist $\delta_{i}$ increasing to $\delta_{0}$ such that

$$
\tilde{X}_{\text {cut }}^{\delta_{1}, R}=\tilde{X}_{\text {cut }}^{\delta_{i}, R} .
$$


To prove the lemma, we proceed by contradiction, assuming

$$
\tilde{X}_{\text {cut }}^{\delta_{0}, R} \neq \tilde{X}_{\text {cut }}^{\delta_{1}, R} \text {. }
$$

Then there is a curve $C$ which lifts closed to $\tilde{X}_{\text {cut }}^{\delta_{0}, R}$ but open to $\tilde{X}_{\text {cut }}^{\delta_{1}, R}$. Then $C$ is $\delta_{0}$ homotopic to a collection of curves outside $\bar{B}(x, R)$. Applying Lemma 3.5 we know that for $\delta_{i}$ sufficiently close to $\delta_{0}, H$ is $\delta_{i}$ homotopy. So $C$ lifts closed to $\tilde{X}_{c u t}^{\delta_{i}, R}$. By (4.6) $C$ lifts closed to $\tilde{X}_{c u t}^{\delta_{1}, R}$, which is a contradiction.

4.2. The cut-off $\delta$ covers and $\operatorname{CovSpec}_{c u t}(X)$. We now introduce a cover which will later prove to be basepoint independent whenever it is well defined:

Definition 4.7. The cut-off $\delta$ cover of $X$, denoted $\tilde{X}_{\text {cut }}^{\delta}$, is the Gromov-Hausdorff limit of the $R$ cut-off $\delta$ covers as $R \rightarrow \infty$.

We do not claim that the cut-off $\delta$ cover is defined for an arbitrary metric space, but in the next proposition [Proposition 4.8 we prove they do exist for complete locally compact length spaces. We believe they exist for a much larger class of spaces but will not be investigating this question ourselves. For a review of GromovHausdorff convergence see Section 5 .

Note that as in the case with the cylinder, whose $R$ cut-off $\delta$ covers are all just the cylinder itself; the cut-off $\delta$ cover is also just the cylinder. This is in contrast with the $\delta$ cover which is a Euclidean space for small enough values of $\delta$.

Proposition 4.8. For any complete locally compact length space, the GromovHausdorff limit of the $R$ cut-off $\delta$ covers as $R \rightarrow \infty$ exists and does not depend on the base point $x$. Furthermore we have the following covering maps:

$$
\tilde{X}^{\delta} \mapsto \tilde{X}_{c u t}^{\delta} \mapsto \tilde{X}_{\text {cut }}^{\delta, R} .
$$

Proof. First we fix a base point $x \in X$. By Lemma 4.2 we have a sequence of covering maps

$$
f_{R}: \tilde{X}^{\delta} \rightarrow \tilde{X}_{c u t}^{\delta, R}
$$

and a sequence of covering maps

$$
h_{R}: \tilde{X}_{\text {cut }}^{\delta, R} \rightarrow X
$$

both of which are isometries on balls of radius $\delta$.

Let the maximal number of disjoint balls of radius $\epsilon$ in a ball of radius $r$ in a space $Y$ be denoted $N(\epsilon, r, Y)$. As discussed in Section 2.1, when $X$ is a complete locally compact length space, so is $\tilde{X}^{\delta}$, and so closed balls in $\tilde{X}^{\delta}$ are compact. Thus $N\left(\epsilon, r, \tilde{X}^{\delta}\right)<\infty$. By the covering maps we then have

$$
N\left(\epsilon, r, \tilde{X}_{\text {cut }}^{\delta, R}\right) \leq N\left(\epsilon, r, \tilde{X}^{\delta}\right),
$$

so by Gromov's Compactness Theorem, a subsequence $\tilde{X}_{c u t}^{\delta, R_{j}}$ converges. We call the limit $\tilde{X}_{\text {cut }}^{\delta}$.

Furthermore, by the Grove-Petersen Arzela-Ascoli Theorem, subsequences of $f_{R_{j}}$ and $h_{R_{j}}$ converge to functions $f$ and $h$ such that

$$
\begin{gathered}
f: \tilde{X}^{\delta} \rightarrow \tilde{X}_{c u t}^{\delta}, \\
h: \tilde{X}_{c u t}^{\delta} \rightarrow X,
\end{gathered}
$$

which are still isometries on balls of radius $\delta / 2>0$ and are thus covering maps. In fact they are regular covers. This implies that any limit space satisfies (4.8). 
To show we have a unique limit that doesn't depend on the base point or the sequence $R_{j} \rightarrow \infty$, take an alternate base point $x^{\prime}$ and an alternate sequence $R_{j}^{\prime} \rightarrow \infty$. By the above, a subsequence converges to some other pointed limit space $\left(Z^{\prime}, z^{\prime}\right)$. If we call our original limit $(Z, z)$, we now prove $Z$ is isometric to $Z^{\prime}$.

Taking a subsequence so that

$$
B_{x}\left(R_{j}\right) \subset B_{x^{\prime}}\left(R_{j}^{\prime}\right) \subset B_{x}\left(R_{j+1}\right)
$$

and applying Lemma 4.3 , we have covering maps

$$
\begin{gathered}
f_{j}: \tilde{X}_{c u t}^{\delta, R_{j+1}} \rightarrow \tilde{X}_{c u t}^{\delta, R_{j}^{\prime}}, \\
h_{j}: \tilde{X}_{c u t}^{\delta, R_{j}^{\prime}} \rightarrow \tilde{X}_{c u t}^{\delta, R_{j}},
\end{gathered}
$$

which are isometries on $\delta$ balls. Subsequences converge by the Grove-Petersen Arzela-Ascoli Theorem to covering maps

$$
f_{\infty}: Z \rightarrow Z^{\prime} \text { and } h_{\infty}: Z^{\prime} \rightarrow Z \text {. }
$$

Note further that $h_{\infty}\left(f_{\infty}(z)\right)=z$ and that $h_{\infty} \circ f_{\infty}$ is an isometry on balls of radius $\delta / 3$, so it is a covering map.

If $f_{\infty}$ and $h_{\infty}$ do not form an isometry and its inverse, then we may assume without loss of generality that $f_{\infty}$ is not a one-to-one cover and there exists $w$ such that $f_{\infty}(z)=f_{\infty}(w)$. Let $\gamma_{1}$ be a minimizing geodesic between $z$ and $w$ of length $L=d_{Z}(z, w)$.

Note that by Hopf-Rinow, the closed ball $B(z, L)$ is compact. Thus we can lift $\gamma_{1}:[0, L] \rightarrow B(z, L)$ to a curve $\gamma_{2}:[0, L] \rightarrow B(z, L)$ such that $\gamma_{2}(0)=z$, $L\left(\gamma_{2}\right)=L$ and $h_{\infty}\left(f_{\infty}\left(\gamma_{2}(t)\right)\right)=\gamma_{1}(t)$. We may repeatedly lift the curves to obtain $\gamma_{j}:[0, L] \rightarrow B(z, L)$ such that $\gamma_{j}(0)=z, L\left(\gamma_{j}\right)=L$ and $h_{\infty}\left(f_{\infty}\left(\gamma_{j}(t)\right)\right)=\gamma_{j-1}(t)$. By the compactness of $B(z, L)$ we know that a subsequence of the points $\gamma_{j}(L)$ must converge. In particular, there exists $k>l \in \mathbb{N}$ such that

$$
d=d_{Z}\left(\gamma_{k}(L), \gamma_{l}(L)\right)<\delta / 3
$$

Since $h_{\infty} \circ f_{\infty}$ is an isometry on balls of radius $\delta / 3$, we may then apply this map arbitrarily many times, and the images of these two points will still be a distance $d$ apart. Thus

$$
d=d_{Z}\left(\left(h_{\infty} \circ f_{\infty}\right)^{k-1} \circ \gamma_{k}(L),\left(h_{\infty} \circ f_{\infty}\right)^{k-1} \circ \gamma_{l}(L)\right)=d_{Z}(z, w)
$$

and

$$
d=d_{Z}\left(\left(h_{\infty} \circ f_{\infty}\right)^{k} \circ \gamma_{k}(L),\left(h_{\infty} \circ f_{\infty}\right)^{k} \circ \gamma_{l}(L)\right)=d_{Z}(z, z)=0 .
$$

Thus $z=w$ and we have an isometry between $Z$ and $Z^{\prime}$, and $\tilde{X}_{c u t}^{\delta}$ is uniquely defined.

We leave the following proposition as an exercise, as it can be proven using similar limits of covering maps:

Proposition 4.9. For all $\delta_{1}<\delta_{2}$ we have

$$
\tilde{X}_{c u t}^{\delta_{1}} \mapsto \tilde{X}_{c u t}^{\delta_{2}}
$$

Definition 4.10. The cut-off covering spectrum, denoted $\operatorname{CovSpec}_{\text {cut }}(X)$, is the collection of $\delta>0$ such that

$$
\tilde{X}_{c u t}^{\delta_{1}} \neq \tilde{X}_{c u t}^{\delta}
$$

for all $\delta_{1}>\delta$. 
This spectrum is defined for any metric space which has well defined cut-off $\delta$ covers for all values of $\delta>0$. Note for example that for any simply connected covering space, $X$, we have $\tilde{X}_{c u t}^{\delta, R}=X$ for all $R$ and $\delta$, and so $\tilde{X}_{c u t}^{\delta}=X$ for all $\delta$ and $\operatorname{CovSpec}_{\text {cut }}(X)$ is well defined and empty. The same thing occurs when $X$ is the standard cylinder, $S^{1} \times \mathbb{R}$.

By Proposition 4.8, $\operatorname{CovSpec}_{\text {cut }}(X)$ is well defined for all complete locally compact length spaces, $X$, as well. We expect it is well defined for a much larger class of spaces but will not be pursuing that investigation ourselves. See Theorem 4.23 and Examples 4.34 and 4.36 for other settings where the cut-off covering spectrum is well defined.

Note that by Proposition 4.9, Theorem 7.5] and this definition, we have:

Lemma 4.11. The cut-off covering spectrum is a lower semiclosed set.

The following proposition is easy to prove from the definitions.

Proposition 4.12. If $X$ is a bounded metric space with $D=\operatorname{diam}(X)$, then

$$
\tilde{X}_{c u t}^{\delta, R}=\tilde{X}^{\delta} \quad \forall R \geq D, \text { and } \tilde{X}_{c u t}^{\delta}=\tilde{X}^{\delta} .
$$

So $\operatorname{CovSpec}_{\text {cut }}(X)=\operatorname{CovSpec}(X)$.

Thus the cut-off covering spectrum is really only useful to study complete length spaces which are not bounded.

In the next subsection we explore the distinction between these two spectra in general.

4.3. Relating the various spectra. The intuitive idea behind the next theorem is that the covering spectrum can detect any holes that the cut-off covering spectrum sees.

Theorem 4.13. The cut-off covering spectrum of a complete locally compact length space is a subset of its covering spectrum.

This follows from Lemma 4.14 and Proposition 4.17 which we state and prove below.

Lemma 4.14. For any basepoint, $x$, in a metric space, $X$,

$$
\operatorname{CovSpec}_{\text {cut }}^{R}(X, x) \subset \operatorname{Cov} \operatorname{Spec}(X)
$$

and

$$
\operatorname{CovSpec}_{\text {cut }}^{R_{1}}(X, x) \subset \operatorname{CovSpec}_{\text {cut }}^{R_{2}}(X, x) \text { for } R_{1}<R_{2} .
$$

Proof. If $\delta \in \operatorname{CovSpec}_{\text {cut }}^{R}(X)$, then $\tilde{X}_{\text {cut }}^{\delta_{1}, R} \neq \tilde{X}_{\text {cut }}^{\delta, R}$ for all $\delta_{1}>\delta$. So there is a nontrivial loop $\gamma$ which lifts to $\tilde{X}_{c u t}^{\delta, R}$ nontrivially and lifts to $\tilde{X}_{c u t}^{\delta_{1}, R}$ trivially. In particular we can choose $\gamma$ which lies in a ball of radius $\delta_{1}$. Otherwise if all such loops lift trivially to $\tilde{X}_{\text {cut }}^{\delta, R}$, then the covering groups are the same.

If $\delta \notin \operatorname{CovSpec}(X)$, then $\tilde{X}^{\delta}=\tilde{X}^{\delta_{1}}$ for some $\delta_{1}>\delta$. Then $\gamma$, which lifts trivially to the $\delta_{1}$ cover, also lifts trivially to the $\delta$ cover, and it must then project trivially back down to $\tilde{X}_{c u t}^{\delta, R}$ nontrivially. causing a contradiction.

Similarly if $\delta \notin \operatorname{CovSpec} \operatorname{Sut}_{\text {cut }}^{R_{2}}(X)$, then $\tilde{X}_{\text {cut }}^{\delta, R_{2}}=\tilde{X}_{\text {cut }}^{\delta_{1}, R_{2}}$ for some $\delta_{1}>\delta$, and we can lift $\gamma$ trivially to both of these covers, which contradicts the fact that it lifts to $\tilde{X}_{\text {cut }}^{\delta, R}$ nontrivially. 
Proposition 4.15. If $X$ is a complete locally compact length space, then for any basepoint $x \in X$,

$$
\bigcup_{R>0} \operatorname{CovSpec}_{c u t}^{R}(X, x) \subset \operatorname{CovSpec}_{c u t}(X) .
$$

Proof. If $\delta \in \operatorname{CovSpec} R_{c u t}^{R_{0}}(X)$, by (4.25), then $\delta \in \operatorname{CovSpec}_{\text {cut }}^{R}(X)$ for all $R \geq R_{0}$. So the covering map

$$
\pi_{R}: \tilde{X}_{\text {cut }}^{\delta, R} \rightarrow \tilde{X}_{\text {cut }}^{\delta_{1}, R}
$$

is nontrivial for all $\delta_{1}>\delta$. Then as $R \rightarrow \infty$, the limit map

$$
\pi: \tilde{X}_{c u t}^{\delta} \rightarrow \tilde{X}_{c u t}^{\delta_{1}}
$$

is nontrivial. So $\delta \in \operatorname{CovSpec}_{\text {cut }}(X)$. Hence

$$
\bigcup_{R>0} \operatorname{CovSpec}_{\text {cut }}^{R}(X) \subset \operatorname{CovSpec}_{\text {cut }}(X) .
$$

At first one might think that the inclusion in (4.26) is equal. This is not true.

Example 4.16. Let $X$ be a line with circles attached at the integers $j \neq 0$ of circumference $2 \pi r_{j}$, where $r_{j}=1+1 /|j|$. Using 0 as the base point we have

$$
\operatorname{CovSpec}_{\text {cut }}^{R}(X)=\{\pi+\pi / j: j \in \mathbb{N}, j+1 \leq R\},
$$

because $R$ cut-off $\delta$ covers unravel all loops such that $j+1 \leq R$ and $\pi+\pi / j \geq \delta$. Taking the Gromov-Hausdorff limit of these covers we see that the cut off $\delta$ covers of $X$ unravel all loops $\pi+\pi / j \geq \delta$. Thus $\operatorname{CovSpec}_{c u t}(X)$ is the lower semiclosure of $\{\pi+\pi / j: j \in \mathbb{N}, j+1 \leq R\}$ which includes the number $\pi$, because for all $\delta^{\prime}>\pi$ we have $\tilde{X}_{c u t}^{\delta^{\prime}} \neq \tilde{X}_{c u t}^{\pi}$. However the union of $\operatorname{Cov} \operatorname{Spec}_{c u t}^{R}(X)$ over all $R>0$ does not include the number $\pi$.

Proposition 4.17. If $X$ is a complete locally compact length space, then the lower semiclosure of the union of all $R$ cut-off spectra is the cut-off covering spectrum:

$$
C l_{\text {lower }}\left(\bigcup_{R>0} \operatorname{CovSpec}_{\text {cut }}^{R}(X)\right) \cup\{0\}=\operatorname{CovSpec}_{\text {cut }}(X) \cup\{0\} .
$$

Proof. Take the the lower semiclosure to both sides of (4.26). Since $\operatorname{CovSpec}_{\text {cut }}(X)$ is lower semiclosed by Theorem 7.5, we have

$$
\operatorname{Cl}_{\text {lower }}\left(\bigcup_{R>0} \operatorname{CovSpec}_{\text {cut }}^{R}(X)\right) \subset \operatorname{CovSpec}_{\text {cut }}(X) .
$$

Now suppose $\delta>0$ is not in the lower semiclosure of $\bigcup_{R>0} \operatorname{CovSpec}_{c u t}^{R}(X)$. Then by Lemma 7.4 there exists $\epsilon>0$ such that

$$
[\delta, \delta+\epsilon) \cap \bigcup_{R>0} \operatorname{CovSpec}_{c u t}^{R}(X)=\emptyset .
$$

So for all $R>0$,

$$
[\delta, \delta+\epsilon) \cap \operatorname{Cov} \operatorname{Spec}_{c u t}^{R}(X)=\emptyset,
$$

which implies (by Lemma 4.6) that

$$
\tilde{X}_{c u t}^{\delta+\epsilon, R}=\tilde{X}_{c u t}^{\delta, R} .
$$


Taking the $R \rightarrow \infty$ and the Gromov-Hausdorff limits of these spaces, we get

$$
\tilde{X}_{c u t}^{\delta+\epsilon}=\tilde{X}_{c u t}^{\delta},
$$

which implies that $\delta \notin \operatorname{CovSpec}_{\text {cut }}(X)$.

Proof of Theorem 4.13. Combining (4.24) with Proposition 4.17, the result follows since $\operatorname{Cov} \operatorname{Spec}(X)$ is a lower semiclosed set.

4.4. The length spectrum and the cut-off spectrum. We now relate the cutoff covering spectrum to the length spectrum of a complete locally compact length space. Recall that Example 4.16 is such a space.

Theorem 4.18. If $X$ is a complete locally compact length space, then

$$
\operatorname{CovSpec}_{\text {cut }}^{R}(X) \subset(1 / 2) L(X) .
$$

That is, if $\delta \in \operatorname{CovSpec}_{\text {cut }}^{R}(X)$, then $2 \delta \in L(X)$.

The assumption that the space be locally compact is necessary:

Example 4.19. Let $X$ be the collection of circles of circumference $2 \pi+2 \pi / k$. Then

$$
\operatorname{CovSpec}_{\text {cut }}(X)=\operatorname{CovSpec}(X)=\{\pi+\pi / k: k \in \mathbb{N}\} \cup\{\pi\},
$$

while the $(1 / 2)$ length spectrum of the collection of circles is all finite sums:

$$
(1 / 2) \operatorname{Length}(X)=\left\{\sum_{k=1}^{\infty} a_{k} \pi(1+1 / k): a_{k} \in \mathbb{N}\right\}
$$

which does not include $\pi$.

Before we prove Theorem 4.18, we prove the corresponding proposition which does not require local compactness:

Proposition 4.20. Let $X$ be a complete locally compact length space. If we have $\delta \in \operatorname{CovSpec}_{\text {cut }}^{R}(X, x)$, then there exist $\delta_{j}$ decreasing to $\delta$ and loops, $\sigma_{j}$, with $L\left(\sigma_{j}\right)<2 \delta_{j}$, which are not $\delta$ homotopic to a collection of loops lying outside $\bar{B}(x, R)$.

Proof of Proposition 4.20, Given $\delta \in \operatorname{CovSpec}_{c u t}^{R}(X, x)$ we know there exists $\delta_{j}$ decreasing to $\delta$ such that

$$
\tilde{X}_{\text {cut }}^{\delta, R} \neq \tilde{X}_{\text {cut }}^{\delta_{j}, R} .
$$

So there exist loops $C_{j}$ in $X$ which are $\delta_{j}$ homotopic to loops outside $\bar{B}_{x}(R)$ but are not $\delta$ homotopic to such a curve. Note that $C_{j}$ is homotopic to a combination of curves $\alpha \beta \alpha^{-1}$, where $\beta$ lie outside $\bar{B}_{x}(R)$ or inside $B_{p}\left(\delta_{j}\right)$. If all the $\beta$ curves lie outside $\bar{B}_{x}(R)$, then $C_{j}$ is $\delta$ homotopic to such curves, so this is impossible. In fact there must be a $\beta_{j}$ which lies in a ball $B_{p_{j}}\left(\delta_{j}\right)$ which is not $\delta$ homotopic to a collection of loops outside $\bar{B}_{x}(R)$.

By Lemma $8.2 \beta_{j}$ is freely homotopic to a collection of curves of length $<2 \delta_{j}$. At least one of these curves is not $\delta$ homotopic to a collection of loops outside $\bar{B}_{x}(R)$ because $\beta_{j}$ is not. This is the loop $\sigma_{j}$. 
We can now add the condition that the space is locally compact:

Proof of Theorem 4.18. By Proposition 4.20 we have a sequence of curves $\sigma_{j}$ in $X$. Note that $\operatorname{Im}\left(\sigma_{j}\right) \cap \bar{B}_{x}(R)$ is nonempty for all $j$. Since $L\left(\sigma_{j}\right)<2 \delta_{j}<4 \delta$ for $j$ sufficiently large,

$$
\sigma_{j}:\left[0, L\left(\sigma_{j}\right)\right] \rightarrow \bar{B}_{x}(R+2 \delta) .
$$

By the local compactness this closed ball is compact for $j$, so we can apply the Arzela-Ascoli Theorem to produce a converging subsequence and a limit loop $\sigma_{\infty}$.

It is easy to construct a $\delta$ homotopy from $\sigma_{\infty}$ to $\sigma_{j}$ for $j$ sufficiently large so that $\sigma_{\infty}$ is also not $\delta$ homotopic to a loop outside $\bar{B}_{x}(R)$ and, in particular, not $\delta$ homotopic to a point. Since

$$
L\left(\sigma_{\infty}\right) \leq \liminf _{i \rightarrow \infty} L\left(\sigma_{i}\right) \leq \liminf _{i \rightarrow \infty} 2 \delta_{i}=2 \delta
$$

we can apply Lemma 3.2 to say that $\sigma_{\infty}$ is a closed geodesic and has length $2 \delta$, so $2 \delta \in L(X)$.

Combining this with Proposition 4.17, we get

Corollary 4.21. For a complete locally compact length space $X$,

$$
\operatorname{CovSpec}_{\text {cut }}(X) \subset(1 / 2) \operatorname{Cl}_{\text {lower }}(L(X)) .
$$

That is, if $h / 2 \in \operatorname{CovSpec} R$ cut $(X)$, then either $h \in L(X)$ or there exist $h_{j} \in L(X)$ such that $h_{j}$ decrease to $h$.

Example 4.16 shows that the lower semiclosure is needed here. In Section 4.7 we will see that there are complete length spaces which are not locally compact, with well defined cut-off covering spectra which are not in the closure of the length spectrum.

4.5. Topology and the $\operatorname{CovSpec}_{\text {cut }}(X)$. In this section we prove that the cut-off covering spectrum is empty, given certain topological conditions on a metric space $X$ : particularly Theorem 4.23 and its converse and Theorem 4.26, Recall that the covering spectrum of a simply connected compact metric space is empty, while the cut-off covering spectrum of a cylinder is empty. We begin with the loops to infinity property defined in $[\mathrm{So}]$ :

Definition 4.22. Given a metric space, $X$, a loop $\gamma: S^{1} \rightarrow X$ is said to have the loops to infinity property, if for every compact set $K \subset X$, there is another loop $\sigma: S^{1} \rightarrow X \backslash K$ freely homotopic to $\gamma$.

The space $X$ is said to have the loops to infinity property if all its noncontractible loops have this property.

Theorem 4.23. Any metric space $X$ with the loops to infinity property has a well defined but empty cut-off covering spectrum.

Proof. Fix $x_{0} \in X$ and $\delta>0$. For every $R>0$ let $K=B_{x_{0}}(R)$, and for any $g \in \pi_{1}\left(X, x_{0}\right)$ let $\gamma$ be a representative of $g$ based at $x_{0}$. So there exists $\beta$ freely homotopic to $\gamma$ outside $K$, which means there is a curve $\alpha \circ \beta \circ \alpha^{-1}$ which represents $g$ such that $\beta \subset X \backslash \bar{B}_{p}(R)$. So every $g \in \pi_{1}\left(X, x_{0}\right)$ is in the covering group of $\tilde{X}_{\text {cut }}^{\delta, R}$, which means $\tilde{X}_{\text {cut }}^{\delta, R}=X$. Taking the limit $R \rightarrow \infty$ we get $\tilde{X}_{\text {cut }}^{\delta}=X$ for all $\delta$, so the cut-off covering spectrum is well defined but trivial. 
This theorem is applied to complete manifolds with nonnegative Ricci curvature in Theorem 6.6. Such manifolds have only one end.

Recall that a length space $X$ is said to have $k$ ends if for all sufficiently large compact sets $K, X \backslash K$ has $k$ path connected components.

A length space is semilocally simply connected if every point has a neighborhood around it such that any curve in that neighborhood is contractible. A Riemannian manifold is semilocally simply connected.

Theorem 4.24. Let $X$ be a complete, locally compact and semilocally simply connected length space with an empty cut-off covering spectrum. Then any curve in $X$ is homotopic to a product of curves which have the loops to infinity property. If in addition $X$ has only one end, then $X$ has the loops to infinity property.

Example 4.25 below, demonstrates the necessity of the one end hypothesis, while Example 4.36 demonstrates the necessity of the local compactness condition.

Proof. If the cut-off covering spectrum is empty, then $\tilde{X}_{\text {cut }}^{\delta}=X$ for all $\delta>0$ and, by Proposition 4.8, $\tilde{X}_{\text {cut }}^{\delta, R}$ is between these two spaces, so it is isometric to $X$ as well. Thus for all $\delta>0$ and for all $R>0$, the fundamental group of $X$ is generated by elements of the form $\alpha \circ \beta \circ \alpha$, where $\beta$ is either in a ball of radius $\delta$ or in $X \backslash \bar{B}_{x_{0}}(R)$.

Choose any nontrivial loop $\gamma$ and any compact set $K \subset X$. Take $R>0$ large enough that

$$
K \cup \operatorname{Im}(\gamma) \subset B_{x_{0}}(R / 2) .
$$

Since $X$ is complete, locally compact and semilocally simply connected, we can take $\delta>0$ small enough that balls of radius $\delta$ in $B_{x_{0}}(R)$ are semilocally simply connected so that any loop $\beta$ in such a ball is contractible. Thus $[\gamma] \in \pi_{1}\left(X, x_{0}\right)$ must be generated by loops of the form $\alpha \circ \beta \circ \alpha^{-1}$, where

$$
\beta \in X \backslash \bar{B}_{x_{0}}(R) \subset X \backslash K \text {. }
$$

When $X$ has only one end, the set $X \backslash K$ is path connected. Thus the various $\beta$ used to generate $X$ can be connected via new paths $\alpha \in X \backslash K$ to a point $x_{1} \in X \backslash K$. Thus we have constructed $\sigma \in X \backslash K$, which is freely homotopic to $\gamma$.

Example 4.25. One end is necessary, as can be seen by taking the length space $X$ formed by joining two closed half cylinders at a point. The loop $\gamma$, running around a figure eight which goes once around each cylinder, does not have the loops to infinity property. It is generated by 2 different loops $\beta_{j}$, each of which goes to infinity in a different direction. This can be made smooth by taking the connected sum of two manifolds that are not simply connected that have only one end each, such as Nabonnand's example $\mathrm{Na}$.

Theorem 4.26. If a complete length space $X$ is homeomorphic to the product of complete locally compact length spaces, $M \times N$, then $X$ has the loops to infinity property and CovSpec cut $(X)=\emptyset$ if either of the following holds:

i) both $M$ and $N$ are noncompact,

ii) $M$ is noncompact and $\operatorname{Cov} \operatorname{Spec}(M)=\emptyset$.

Proof. Let $C$ be a loop in $X$, so $C=(a, b)$ where $a$ and $b$ are closed loops in $M$ and $N$, respectively. $C$ is freely homotopic to $\left(a, b_{0}\right)$ followed by $\left(a_{0}, b\right)$, where $b_{0}=b(0)$ and $a_{0}=a(0)$. 
In both cases $M$ is complete and noncompact, so there exists $p_{j} \in M$ which diverge to infinity and there exist minimal paths $\sigma_{j}$ from any fixed point $p_{0}$ to $p_{j}$. If $b$ is a loop in $N$, then $\left(p_{0}, b\right)$ is freely homotopic to $\left(p_{i}, b\right)$ via $\left(\sigma_{j}, b\right)$. Any compact $K \subset X$ is a subset of the image of $K_{M} \times K_{N}$, where $K_{M}$ is compact in $M$. Taking $p_{j} \in M \backslash K_{M}$ we have $\left(p_{j}, b\right)$ outside $K$. Thus $\left(p_{0}, b\right)$ has the loops to infinity property.

In case i), $N$ is also noncompact, so both $\left(a, b_{0}\right)$ and $\left(a_{0}, b\right)$ have the loops to infinity property. So any loop $C$ in $X$ is a combination of curves with the loops to infinity property, and we just apply Theorem 4.23 .

Before we begin case ii) we note that: if a has the loops to infinity property, then so does $\left(a, b_{0}\right)$. This is seen by taking the homotopies $h_{i}$ from $a$ to $a_{i}$ that diverge to infinity. Mapping them to $X$, we get homotopies $\left(h_{i}, b_{0}\right)$ from $\left(a, b_{0}\right)$ to $\left(a_{i}, b_{0}\right)$. So for any compact set $K \subset X$, we have $K \subset K_{M} \times K_{N}$, where $K_{M}$ is compact. So we can choose $a_{i}$ in $M \backslash K_{M}$ and have $\left(a_{i}, b_{0}\right)$ outside $K$.

In case ii), we don't have a ray in $N$ for the loops in $M$, but $\operatorname{CovSpec}_{\text {cut }}(M)=\emptyset$. Applying Theorem 4.24, we see that the loop $a$ in $M$ is freely homotopic to a combination of loops which have the loops to infinity property. Thus $(a, b)$ is freely homotopic to a combination of loops $\left(a_{i}, b\right)$, each of which is homotopic to $\left(a_{i}(0), b\right)$ following $\left(a_{i}, b(0)\right)$. Each $\left(a_{i}, b(0)\right)$ has the loops to infinity property via the loops to infinity property of each $a_{i}$. As in case i), each $\left(a_{i}(0), b\right)$ has the loops to infinity property via rays in $M$ based at $a_{i}(0)$. So $\operatorname{CovSpec}_{c u t}(X)=\emptyset$ here as well.

Corollary 4.27. If $X$ is a complete noncompact locally compact length space homeomorphic to $M \times \mathbb{R}$, then

$$
\operatorname{CovSpec}_{\text {cut }}(X)=\emptyset \text {. }
$$

4.6. Pulled ribbon spaces. In this section we construct examples of metric spaces with well defined cut-off covering spectra demonstrating that Theorem 4.18 does not hold without the assumption of local compactness. We call the method of construction the "pulled ribbon construction". It is similar to an idea of Burago, Burago, and Ivanov called a "pulled string", where a collection of points lying on a path in a space is identified creating a new complete length space. Their construction is called the pulled string construction because it looks something like a cloth which has had a thread pulled tight. In our case we first attach a ribbon along the line in the space and then pull a string on the opposite edge of the ribbon.

We will make our construction precise. Those who wish to understand their construction may consult [BBI].

Definition 4.28. The pulled ribbon space is a space $Y=\mathbb{R} \times[0,1]$ with the lower boundary $\mathbb{R} \times\{0\}$ identified with a point and endowed with the induced length structure. This is the same as saying that the metric on $Y$ is

$$
d_{Y}\left(\left(r_{1}, s_{1}\right),\left(r_{2}, s_{2}\right)\right)=\min \left\{\sqrt{\left(r_{1}-r_{2}\right)^{2}+\left(s_{1}-s_{2}\right)^{2}}, s_{1}+s_{2}\right\} .
$$

This is a quasimetric and becomes a metric when we make the identification $\left(r_{1}, 0\right)=$ $\left(r_{2}, 0\right)$.

Note that $Y$ is the suspension of a line. There is a geodesic $\gamma: \mathbb{R} \rightarrow Y$ which runs along the "top edge": $\gamma(r)=(r, 1)$. This geodesic is not a line in the induced length structure. 
Proposition 4.29. The pulled ribbon space is a bounded complete length space which is not locally compact.

Proof. It is bounded because $Y \subset \bar{B}_{y}(1)$, where $y$ is the special identified point. It is not compact because the sequence of points $(2 j, 1)$ are all a distance 2 apart from each other. It is a complete length space because between any pair of points we can find a minimal geodesic between them: it is either the line segment in the strip or a pair of vertical lines dropping from the points to the common point. Given any Cauchy sequence $\left(r_{i}, s_{i}\right)$ in the induced length structure, either the sequence converges in the standard metric on the strip or it approaches the bottom edge which is the common point.

Definition 4.30. Given a manifold $M$ with a line $\gamma: \mathbb{R} \rightarrow M$, we say that we attach a pulled ribbon to $M$ creating a space, $M_{\gamma}$, if we attach the pulled ribbon so that its top edge is identified with the line. Then we endow $M_{\gamma}$ with the induced length metric.

Note that in this induced length metric the original line $\gamma$ is no longer a line and is now bounded. However, unlike spaces with a pulled thread, a space with an attached pulled ribbon keeps its topology. In fact:

Proposition 4.31. If $x, y \in M$ and $d_{M}(x, y)<2$, then after adding the pulled ribbon to $M$, we do not change the distance between $x$ and $y$.

Proof. If the distance between $x$ and $y$ has been shortened, then there is a path from $x$ to $y$ of length $<2$ which passes into the ribbon. However, such a short path could not reach the far edge of the ribbon, and so its length is determined by the Euclidean structure on the ribbon. It would be shorter if it did not enter the ribbon at all.

Corollary 4.32. Given a space $M$ and a map $f: X \rightarrow M$, then $f$ is continuous from $X$ to $M$ iff $f$ is continuous from $X$ to $M_{\gamma}$ with the ribbon attached.

Corollary 4.33. $M$ is simply connected iff $M_{\gamma}$ is simply connected. Furthermore, $M$ is semilocally simply connected iff $M_{\gamma}$ is semilocally simply connected.

The following example demonstrates the necessity of the local compactness condition in Theorem 4.24,

Example 4.34. Let $M^{2}$ be the cusped manifold,

$$
\mathbb{R} \times_{f} S^{1},
$$

where $f(r)=2 \operatorname{Arctan}(-r)+\pi$, and let $\gamma$ be any line in this space.

Then $M_{\gamma}$ is not simply connected but is semilocally simply connected by Corollary 4.33. It is a bounded space which is not locally compact. Note that $\tilde{M}_{c u t}^{\delta, R}=M$ for every value of $R, \delta>0$. So $\operatorname{CovSpec} R(M))$ is trivial and $\operatorname{CovSpec}_{c u t}^{R}(M)$ is well defined and trivial.

Adding a halfline attached at any point would just create a space which does not have the loops to infinity property and has one end. Nevertheless,

$$
\operatorname{CovSpec}_{\text {cut }}\left(M_{\gamma}\right)=\operatorname{CovSpec}_{\text {cut }}^{R}\left(M_{\gamma}\right)=\operatorname{Cov} \operatorname{Spec}\left(M_{\gamma}\right)=\emptyset .
$$

Note that if we just pulled a thread in this example identifying a line to be a point and using the induced length structure, the space would become simply connected: loops shrinking along the cusp would in fact converge to the identified point which 
is the line. The loops in this example do not have a converging subsequence because they are always a fixed distance away from the line.

It should be noted that the double suspension of the Hawaii Ring is a compact space which is not simply connected and yet it is its own universal cover, so its covering spectrum is empty as well $\mathrm{Sp}$.

We next demonstrate that local compactness is necessary in Theorem 4.18,

Proposition 4.35. If the length spectrum of $M$ is empty and there are no geodesics starting and ending perpendicular to the line $\gamma$, then the length spectrum of $M_{\gamma}$ is also empty.

Proof. Suppose on the contrary that there is a closed geodesic $\sigma: S^{1} \rightarrow M_{\gamma}$. If its image lies in $M \subset M_{\gamma}$, then it is also a closed geodesic in $M$ by Proposition 4.31. Since $M$ has an empty length spectrum this cannot be the case, so its image must intersect with the ribbon. The image of a closed geodesic cannot lie completely within the ribbon, because there are no closed geodesics formed using Euclidean line segments. So the geodesic $\sigma$ must enter and leave the ribbon. The only way $\sigma$ can turn around is if it passes through the far edge and comes back. Thus the geodesic must be vertical and must intersect the line $\gamma$ vertically. So the part of $\sigma$ which lies in $M$ contradicts the hypothesis.

This next example demonstrates the necessity of the local compactness condition in Theorem 4.24

Example 4.36. Let $M$ be asymptotically cylindrical,

$$
\mathbb{R} \times_{f} S^{1},
$$

where $f(r)=2 \operatorname{Arctan}(-r)+2 \pi$, and let $\gamma$ be any line in $M$. Then $M_{\gamma}$ is a bounded complete geodesic length space with diameter $D \leq 2+2 \pi$. It has an empty length spectrum by Proposition 4.35 .

Note that for $\delta \leq \pi, \tilde{M}^{\delta}=\tilde{M}$, which is a complete length space that does not have minimizing geodesics joining every pair of points (particularly the lifts of the pulled point). Note also that for $R \geq D$ and $\delta \leq \pi, \tilde{M}_{c u t}^{R, \delta}=\tilde{M}$, while for $R \geq D$ and $\delta>\pi, \tilde{M}_{c u t}^{R, \delta}=M$. Thus the cut-off $\delta$ covers are defined and so is the cut-off covering spectrum. Furthermore,

$$
\operatorname{CovSpec}_{\text {cut }}\left(M_{\gamma}\right)=\operatorname{Cov} \operatorname{Spec}_{\text {cut }}^{D}\left(M_{\gamma}\right)=\operatorname{Cov} \operatorname{Spec}\left(M_{\gamma}\right)=\{\pi\} .
$$

4.7. Localizing the $R$ cut-off covering spectrum. In this section we show that one can compute $\operatorname{Cov} \operatorname{Spec}_{\text {cut }}^{R}(X, x) \cap[0, D]$ of a metric space $X$ using only the information contained in $B(x, r)$ when $r$ is taken sufficiently large [Proposition4.42. In fact we give a precise estimate on $r$ independent of $X$ which will allow us to study sequences of spaces.

Note that there is a complete hyperbolic manifold $M$ of constant sectional curvature -1 such that for any $r$, there exists a contractible curve lying in $B(p, 1)$ which is not homotopically trivial in $B(p, r)[\mathrm{BoMe},[\mathrm{Po}$. In other words, the homotopies required to contract these loops to a point extend further and further out in $M$. A simpler example with this property is formed by taking the Hawaii Ring with circles of circumference $1 / k$, attaching a cylinder of length $k$ to the $k^{\text {th }}$ circle and then capping off the cylinder. This is a simply connected space, none of whose balls about the basepoint are simply connected. 
The covering spectrum of these spaces could not be computed using a localization process like the one we obtain here for the $R$ cut-off covering spectrum. It is crucial that we can chop off homotopies as in Figure 4 when computing the $R$ cut-off covering spectrum.

Recall the definition of $\delta$ homotopy in Definition 3.1] Lemma 3.4 and Definition 3.6. Now we define:

Definition 4.37. Two loops $\gamma_{1}, \gamma_{2}$ in a metric space, $X$, are $R$-cut-off $\delta$ homotopic in $X$ if $\pi_{\delta, R}\left(\gamma_{1}\right)=\pi_{\delta, R}\left(\gamma_{2}\right)$, where $\pi_{\delta, R}: \pi(X) \rightarrow \pi(X) / \pi(X, \delta, R)$.

It is not hard to see from the definition of the $R$ cut-off $\delta$ cover that we have the following simpler description which will allow us to apply the lemmas from the section on $\delta$ homotopies to study this new kind of homotopy:

Lemma 4.38. Let $A$ be a subset of a metric space $X$. A loop $\gamma$ is $R$-cut-off $\delta$ homotopic to a point in $A$ iff it is $\delta$ homotopic in $A$ to a collection of loops $\beta_{j}$ lying outside $\bar{B}_{p}(R)$.

Our next lemma will be useful for localizing the $\delta$ homotopies so that we can use compactness to control them.

Lemma 4.39. Given $\delta>0, R>0$, and a loop $C$ in $B(x, R+2 \delta)$ in a metric space, $X$, if $C$ is $\delta$ homotopic in $X$ to a collection of curves $\alpha \beta \alpha^{-1}$, where $\beta$ are in $\delta$-balls or outside $\bar{B}(x, R)$, then $C$ is $\delta$-homotopic in $B(x, R+2 \delta)$ to a collection of curves $\alpha \beta \alpha^{-1}$, where $\beta$ are in $\delta$-balls or outside $\bar{B}(x, R)$. So $C$ is $R$-cut-off $\delta$ homotopic to a point in $\bar{B}(x, R+2 \delta)$.

See Figure 4 where the darker balls are $B(x, R)$ and where the lighter balls are $B(x, R+2 \delta)$.
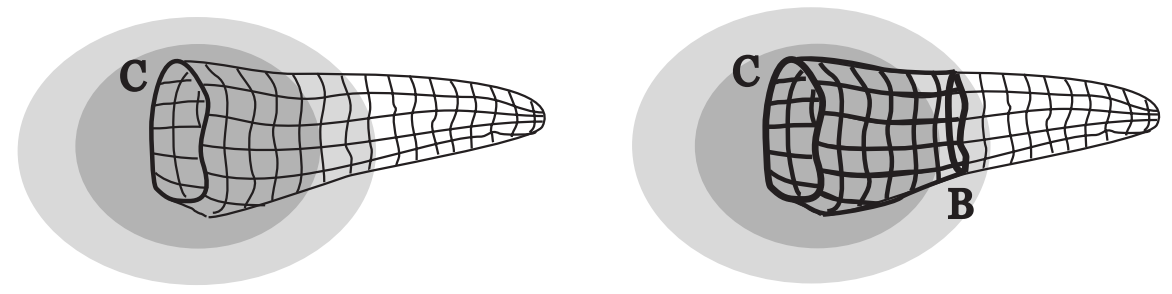

Figure 4. Here $C$ is $\delta$ homotopic to a single $\beta=B$ outside $\bar{B}_{x}(R)$.

Proof. This proof follows from Lemma 3.7 where our sets $A=X$ and $B=$ $B(x, R+2 \delta)$. Therefore, we see that $C$ is $\delta$ homotopic in $B$ to a collection of curves $\gamma_{1}, \gamma_{2}, \ldots, \gamma_{j}$ such that each $\gamma_{j}$ lies in $B$ and in the tubular neighborhood

$$
T_{2 \delta}(A \backslash B) \text {. }
$$

In particular the $\gamma_{i}$ lie outside $\bar{B}(x, R)$. Thus by Lemma 4.38, $C$ is a curve which is $R$-cut-off $\delta$-homotopic in $B(x, R+2 \delta)$ to a collection of curves $\alpha \beta \alpha^{-1}$, where $\beta$ are in $\delta$-balls or outside $\bar{B}(x, R)$.

Using Lemma 4.39 we have the following relation between the $R$-cut-off spectrums of balls and the total space which will be very useful later. 
Lemma 4.40. If $X$ is a metric space, then

$$
\delta \in \operatorname{CovSpec}_{\text {cut }}^{R}(B(x, r))
$$

for some $r>3(R+2 \delta)$. Then

$$
\delta \in \operatorname{CovSpec} \operatorname{cut}_{\text {cut }}^{R}(X) .
$$

When $X$ is a length space we can use the induced length metric on $B(x, r)$ rather than the restricted metric and still have the same result.

Proof. We prove the contrapositive. Assume $\delta \notin \operatorname{CovSpec}_{c u t}^{R}(X)$. By the definition, there exists $\delta^{\prime}>\delta$ such that

$$
\tilde{X}_{\text {cut }}^{\delta, R}=\tilde{X}_{\text {cut }}^{R, \delta^{\prime}} .
$$

This means that any curve $C$ whose image lies in a ball of radius $\delta^{\prime}$ is $\delta$-homotopic in $X$ to a path created as a combination of $\alpha \beta \alpha^{-1}$, where $\beta$ are either in a ball of radius $\delta$ or lie outside $\bar{B}_{x}(R)$.

If the image of $C$ lies in $B\left(x, R+2 \delta^{\prime}\right)$, then by Lemma 4.39 $C$ is a curve which is $\delta$-homotopic in $B\left(x, R+2 \delta^{\prime}\right)$ to a collection of curves $\alpha \beta \alpha^{-1}$, where $\beta$ are in $\delta$-balls or outside $\bar{B}(x, R)$. When $r>3(R+2 \delta)$ and $\delta^{\prime}$ is close to $\delta$, the metric on $B_{x}\left(R+2 \delta^{\prime}\right)$ restricted from the induced length metric on $B_{x}(r)$ agrees with its metric restricted from $X$. We have that any such curve $C$ lifts closed to $\tilde{B}(x, r)_{\text {cut }}^{\delta, R}$.

Now any curve $\sigma$ which lifts closed to $\tilde{B}(x, r)_{\text {cut }}^{R, \delta^{\prime}}$ is homotopic in $B(x, r)$ to a collection of $\alpha \beta \alpha^{-1}$, where $\beta$ is now either in a ball of radius $\delta^{\prime}$ or outside $\bar{B}_{x}(R)$. Note that any $\beta$ which lies outside $\bar{B}_{x}(R)$ lifts as a closed loop to $\tilde{B}(x, r)_{\text {cut }}^{\delta, R}$. For those $\beta$ which pass within $\bar{B}_{x}(R)$ and fit in a ball of radius $\delta^{\prime}$, the images must be contained in $B\left(x, R+2 \delta^{\prime}\right)$, and therefore also for those lifts closed to $\tilde{B}(x, r)_{\text {cut }}^{\delta, R}$. Since $\sigma$ is homotopic in $B(x, r)$ to a combination of curves which lift as closed loops to $\tilde{B}(x, r)_{\text {cut }}^{\delta, R}$, then $\sigma$ must do the same. Thus by the Curve Lifting property (cf. Ma page 123) and Lemma 4.9 we see that

$$
\tilde{B}(x, r)_{c u t}^{\delta^{\prime}, R}=\tilde{B}(x, r)_{c u t}^{\delta, R},
$$

and so $\delta \notin \operatorname{CovSpec} \operatorname{cut}_{\text {e }}^{R}(B(x, r))$.

In the opposition direction we have

Lemma 4.41. If $X$ is a metric space with

$$
\delta \in \operatorname{CovSpec}_{\text {cut }}^{R}(X),
$$

then for all $r \geq 3(R+2 \delta)$,

$$
\delta \in \operatorname{CovSpec}_{\text {cut }}^{R}(B(x, r)) .
$$

Again when $X$ is a length space we may either give $B(x, r)$ the induced length metric or the restricted metric.

Proof. If

$$
\delta \in \operatorname{CovSpec} \operatorname{cut}_{\text {cut }}^{R}(X),
$$

then, for $\delta_{i}$ decreasing to $\delta$, we have loops $C_{i}$ that lie inside $\delta_{i}$ balls of $X$ which cannot be represented in $X$ by loops lying inside $\delta$-balls of $X$ or loops in $X \backslash \bar{B}(x, R)$. Since $C_{i}$ lies inside $\delta_{i}$ balls and is not in $X \backslash \bar{B}(x, R)$, it must be in $B\left(x, R+2 \delta_{i}\right)$. Since $r \geq 3(R+2 \delta)$ the balls in $B\left(x, R+2 \delta_{i}\right)$ are the same for $B(x, r)$ and $X$. Therefore $C_{i}$ lies inside $\delta_{i}$ balls of $B(x, r)$. Since $C_{i}$ cannot be represented in $X$ by 
loops lying inside $\delta$-balls of $X$ or loops in $X \backslash \bar{B}(x, R)$, it cannot be represented by loops lying inside $\delta$-balls of $B(x, r)$ or loops in $B(x, r) \backslash \bar{B}(x, R)$ in $B(x, r)$. This shows that $\delta \in \operatorname{CovSpec} R$ cut $(B(x, r))$.

An immediate consequence of these two lemmas is the following:

Proposition 4.42. Given two metric spaces $X$ and $Y$ with isometric balls, $B(x, r)$ $=B(y, r)$, then

$$
\operatorname{CovSpec}_{\text {cut }}^{R}(X, x) \cap[0, D]=\operatorname{CovSpec}_{\text {cut }}^{R}(Y, y) \cap[0, D]
$$

whenever $3(R+2 D) \leq r$. These balls may have restricted metrics or induced length metrics when $X$ and $Y$ are length spaces.

Proof. If $\delta \in \operatorname{CovSpec}_{c u t}^{R}(X, x) \cap[0, D]$, then $\delta \leq D$, so apply Lemma 4.41 and have

$$
\delta \in \operatorname{CovSpec}_{\text {cut }}^{R}(B(x, r))=\operatorname{CovSpec}_{\text {cut }}^{R}(B(y, r)) .
$$

Then apply Lemma 4.40, which gives the result.

Remember that the $R$ cut-off covering spectrum of a capped cylinder and a cylinder are both empty regardless of basepoint, while the ordinary covering spectrum of the cylinder is nonempty.

Without restricting to a uniform $[0, D]$, the $R$ cut-off covering spectrum will not match. This can be seen in the following example:

Example 4.43. Let $X_{s}$ be a unit interval with $x_{s}$ on one end and a circle of circumference $2 \pi s$ on the other end. Let $Y$ be a unit interval with $y$ at one end and two half lines at the far end. Taking $R=2$ and $s>1$ we have $\operatorname{CovSpec}_{c u t}^{R}\left(X_{s}, x_{s}\right)=$ $\{\pi s\}$ and $\operatorname{CovSpec}_{c u t}^{R}(Y, y)=\emptyset$. Yet for any $r$ we have $B\left(x_{s}, r\right)$ isometric to $B(y, r)$ for $s>r$.

4.8. Varying $R$ in the $R$ cut-off covering spectra. In the next section on the Gromov-Hausdorff convergence of metric spaces and the cut-off covering spectra we need to relate the $R$ cut-off covering spectra for various values of $R$.

Proposition 4.44. Given a metric space $X$ and $R_{0}<R_{1}$,

$$
\delta \in \operatorname{CovSpec}_{\text {cut }}^{R_{1}}(X) \backslash \operatorname{CovSpec}_{\text {cut }}^{R_{0}}(X)
$$

implies

is nontrivial.

$$
\tilde{X}_{\text {cut }}^{\delta, R_{1}} \rightarrow \tilde{X}_{\text {cut }}^{\delta, R_{0}}
$$

Proof. If $\delta \in \operatorname{CovSpec} c_{\text {cut }}^{R_{1}}(X)$, then $\tilde{X}_{\text {cut }}^{\delta_{i}, R_{1}} \neq \tilde{X}_{\text {cut }}^{\delta, R_{1}}$ for all $\delta_{i}>\delta$. So there is a nontrivial loop $\gamma_{i}$ which lifts to $\tilde{X}_{c u t}^{\delta, R_{1}}$ nontrivially and lifts to $\tilde{X}_{c u t}^{\delta_{i}, R_{1}}$ trivially. Since $R_{1}$ is the same for both covering spaces, we can choose $\gamma_{i}$ which lies in a ball of radius $\delta_{i}$. Otherwise if all such loops lift trivially to $\tilde{X}_{c u t}^{\delta, R}$, then the covering groups are the same.

Suppose $\delta \notin \operatorname{Cov} \operatorname{Spec}_{c u t}^{R_{0}}(X)$; then for $i$ sufficiently large,

$$
\tilde{X}_{\text {cut }}^{\delta_{i}, R_{0}}=\tilde{X}_{\text {cut }}^{\delta, R_{0}} .
$$

Since $\gamma_{i}$ lies in a ball of radius $\delta_{i}$ it lifts trivially to the first cover, and thus also the second. So we have a nontrivial covering,

$$
\tilde{X}_{c u t}^{\delta, R_{1}} \rightarrow \tilde{X}_{c u t}^{\delta, R_{0}} .
$$


In the next proposition we assume our space $Y$ is compact. To apply this proposition to complete noncompact spaces $X$ which are only locally compact, we will use our localization results from the last section.

Proposition 4.45. If $Y$ is a compact length space and $\operatorname{CovSpec} \operatorname{sut}_{\text {cut }}^{R_{i}}(Y) \cap\left[\delta_{1}, \delta_{2}\right)=\emptyset$ for a sequence of $R_{i}$ decreasing to $R_{1}$, then for $R_{i}$ sufficiently close to $R_{1}$ we have

$$
\tilde{Y}_{\text {cut }}^{\delta_{1}, R_{i}} \rightarrow \tilde{Y}_{\text {cut }}^{\delta_{2}, R_{1}}
$$

is trivial. In particular, without any assumption on the spectrum, we have

$$
\tilde{Y}_{c u t}^{\delta, R_{i}} \rightarrow \tilde{Y}_{\text {cut }}^{\delta, R_{1}}
$$

is trivial whenever $R_{i}$ is sufficiently close to $R_{1}$.

Combining this with Lemma 4.14, we only need to assume there exists $R_{2}>R_{1}$ with $\operatorname{CovSpec} \mathrm{cut}_{\text {cut }}^{R_{2}}(Y) \cap\left[\delta_{1}, \delta_{2}\right)=\emptyset$ to conclude (4.59). In fact, by Proposition 4.15 we could assume $\operatorname{CovSpec}_{\text {cut }}(Y) \cap\left[\delta_{1}, \delta_{2}\right)=\emptyset$ and draw the same conclusion. See Theorem 5.7 for an application of this proposition.

Proof. Assume on the contrary that $\tilde{Y}^{\delta_{1}, R_{2}} \rightarrow \tilde{Y}^{\delta_{2}, R_{1}}$ is not trivial for all $R_{2}>R_{1}$. So there is a $\gamma$ which lifts trivially to the latter cover, but not to the first. In particular we can either choose $\gamma$ to lie inside a ball of radius $\delta_{2}$ or outside $\bar{B}_{p}\left(R_{1}\right)$.

In the first case $\gamma$ lifts trivially to $\tilde{Y}^{\delta_{2}, R_{2}}$, which implies $\operatorname{Cov} \operatorname{Spec}_{\text {cut }}^{R_{2}}(Y) \cap\left[\delta_{1}, \delta_{2}\right)$ is nonempty.

In the second case $\gamma$ lies outside $\bar{B}_{p}\left(R_{1}\right)$ and is not $\delta_{1}$ homotopic to a loop outside $\bar{B}_{p}\left(R_{2}\right)$. In particular, $l(\gamma) \geq 2 \delta_{1}$ and $\gamma$ is not $\delta$ homotopic to a loop outside $\bar{B}_{p}\left(R_{2}\right)$ for any $\delta \leq \delta_{1}$.

Suppose we take $R_{2}=R_{i}$ decreasing to $R_{1}$ and having nontrivial covers. So we get a sequence of $\gamma_{i}$, where each $\gamma_{i}$ lies outside $\bar{B}_{p}\left(R_{1}\right)$ and is not $\delta_{1}$ homotopic to a loop outside $\bar{B}_{p}\left(R_{i}\right)$.

Note that $Y \backslash \bar{B}_{p}\left(R_{1}\right)$ is precompact. It is still a precompact length space if we give it the induced length structure (cf. [BBI]). So there exists some finite number $N$ such that it can be covered by at most $N$ balls of radius $\delta_{1} / 5$. Note that balls in the induced length metric are smaller than those in the metric on $Z$, so $\gamma_{i}$ is also not $\delta_{1}$ homotopic in the space $Z=Y \backslash \bar{B}_{p}\left(R_{1}\right)$ to a loop outside $\bar{B}_{p}\left(R_{i}\right)$. For the rest of the proof we will use the induced length metric on $Z$ when referring to the $\delta_{1}$ homotopies.

Applying Lemma 3.9, we see that we can always find a $\gamma_{i}$ in $Z$ with $L\left(\gamma_{i}\right) \leq N \delta_{1}$ which is not $\delta_{1}$ homotopic to a loop outside $\bar{B}_{p}\left(R_{i}\right)$.

Since the $\gamma_{i}$ have length bounded above uniformly and since $Y$ is compact, by Arzela Ascoli we have a subsequence which converges to some $\gamma_{\infty}$. Note that $\gamma_{\infty}$ need not be located outside $\bar{B}_{p}\left(R_{1}\right)$, so instead of relating $\gamma_{i}$ to $\gamma_{\infty}$, we will use the fact that $\gamma_{i}$ must be a Cauchy sequence in $Z$. That is, there exists $N^{\prime}$ sufficiently large such that $\gamma_{i}$ are $\delta_{1} / 2$ homotopic to $\gamma_{j}$ for all $i, j \geq N^{\prime}$. Fix this $N$ and note that $\gamma_{N}$ lies outside the closed ball $\bar{B}_{p}\left(R_{1}\right)$ and $R_{j}$ are decreasing to $R_{1}$, so $\gamma_{N}$ is outside $\bar{B}_{p}\left(R_{j}\right)$ for $j$ sufficiently large. This contradicts the fact that $\gamma_{j}$ is not $\delta_{1}$ homotopic to a loop outside $\bar{B}_{p}\left(R_{j}\right)$.

Note that the compactness here is essential, as the following example shows.

Example 4.46. Let $Y$ be the Hawaii Ring with circles of circumference $2 \pi \pm \frac{\pi}{j}$, $\gamma_{j}$, all attached at a point. Take $\delta=\pi / 2, R_{i}=(1+1 / i) \pi$, and $R_{1}=\pi$. Then the cover $\tilde{Y}_{c u t}^{\delta, R_{i}} \rightarrow \tilde{Y}_{\text {cut }}^{\delta, R_{1}}$ is nontrivial for all $i$. This $Y$ is not a compact length space. 


\section{Gromov-HausdorfF CONVERGEnCE}

In SoWei3 we proved that when compact spaces $M_{j}$ converge to a compact limit $M$ in the Gromov-Hausdorff sense, then $\operatorname{CovSpec}\left(M_{i}\right) \cup\{0\}$ converges to $\operatorname{CovSpec}(M) \cup\{0\}$ in the Hausdorff sense as subsets of the real line. In particular, if $M_{j}$ are simply connected, then the limit space has an empty covering spectrum and is its own universal cover.

In the next subsection we provide examples demonstrating that we do not get such a strong result when the spaces are noncompact. In fact the limit space of simply connected $M_{i}$ might be a cylinder [Example 5.3.

In the subsequent sections we prove the continuity of the cut-off covering spectra [Theorem 5.7]. In particular the limit of simply connected manifolds will be seen to have an empty cut-off covering spectrum [Corollary 5.13].

First recall the definition of Gromov-Hausdorff distance:

Definition 5.1. Given compact length spaces $X_{i}$ and $Y$, we say $X_{i}$ converges to $Y$ in the Gromov-Hausdorff sense if there exists $\delta_{i}$ Hausdorff approximations $f_{i}: X_{i} \rightarrow Y$ such that

$$
\left|d_{Y}\left(f_{i}\left(x_{1}\right), f_{i}\left(x_{2}\right)\right)-d_{X_{i}}\left(x_{1}, x_{2}\right)\right|<\delta_{i}
$$

and $Y \subset T_{\delta_{i}}\left(f\left(X_{i}\right)\right)$ with $\delta_{i} \rightarrow 0$. Note that once this is true there are also $\delta_{i}^{\prime}$ Hausdorff approximations from $Y$ to $X_{i}$ with $\delta_{i}^{\prime} \rightarrow 0$.

When complete noncompact spaces are said to converge in the Gromov-Hausdorff sense, they are considered to be pointed spaces. We write that $\left(X_{i}, x_{i}\right)$ converges in the pointed Gromov-Hausdorff sense to $(X, x)$ when for every $R>0$, the closed balls with the restricted metric $\bar{B}_{x_{i}}(R) \subset X_{i}$ converge to balls in the limit space $\bar{B}_{x}(R) \subset X$.

Gromov's Compactness Theorem says that a sequence of complete locally compact metric spaces, $X_{k}$, converges in the pointed Gromov-Hausdorff sense iff they are uniformly locally compact in the sense that the number of disjoint balls of radius $\epsilon$ lying in a ball of radius $R$ is uniformly bounded, $N\left(\epsilon, R, X_{k}\right) \leq N(\epsilon, R)$. Crucial here is that the balls of increasing radius $R$ do piece together to form a complete locally compact limit space which is also a length space when the $X_{k}$ are length spaces $\mathrm{Gr}$. However, one must keep in mind that the balls can converge at different rates. The next section depicts a few examples where this aspect of the pointed Gromov-Hausdorff convergence is crucial.

5.1. Examples. First recall that even for the covering spectrum on compact spaces it is possible for a sequence of spaces to become simply connected in the limit:

Example 5.2. Let $M$ be a simply connected surface and let $X_{k}$ be created by adding a small handle onto $M$, such that the handle fits inside a ball of radius $1 / k$. These $X_{k}$ converge to $M$ as $k \rightarrow \infty$. Note that both $\operatorname{Cov} \operatorname{Spec}\left(X_{k}\right)=\left\{\delta_{k}\right\}$ and $\operatorname{CovSpec}_{\text {cut }}\left(X_{k}\right)=\left\{\delta_{k}\right\}$ while $\operatorname{Cov} \operatorname{Spec}(M)=\emptyset$.

Nevertheless in SoWei3] we proved that the difficulty seen here was the only cause for a lack of continuity in the covering spectrum. We proved that for compact $M_{i}$ converging to compact limits $Y$, if $\lambda_{j} \in \operatorname{CovSpec}\left(M_{i}\right)$ converge to $\lambda>0$, then $\lambda \in \operatorname{CovSpec}(Y)$, and if $\lambda \in \operatorname{CovSpec}(Y)$ there exists $\lambda_{j} \in \operatorname{Cov} \operatorname{Spec}\left(M_{i}\right) \operatorname{such}$ that $\lambda_{j} \rightarrow \lambda$. In particular, if the $M_{i}$ are simply connected, then $Y$ has an empty covering spectrum. 
Without the assumption of compactness, however, we can have simply connected manifolds which have a limit with a nonempty covering spectrum:

Example 5.3. Let $M$ be a capped off cylinder and let $p_{i} \in M$ diverge to infinity. Then the sequence $\left(M_{i}, p_{i}\right)$ converges in the pointed Gromov-Hausdorff sense to a cylinder because the cap has disappeared off to infinity.

Thus we have a sequence $M_{i} \rightarrow Y$ such that $\operatorname{CovSpec}\left(M_{i}\right)=\emptyset \operatorname{but} \operatorname{Cov} \operatorname{Spec}(Y)$ $=\{\pi\}$. Now by Lemma 4.14 and Proposition 4.15, $\operatorname{CovSpec}_{\text {cut }}\left(M_{i}\right)=$ $\operatorname{CovSpec}_{\text {cut }}^{R}\left(M_{i}\right)=\emptyset$ as well. Since a cylinder has the loops to infinity, $\operatorname{CovSpec}_{\text {cut }}(Y)=\operatorname{CovSpec} \operatorname{Sut}_{\text {cut }}^{R}(Y)$ are also empty.

While in the above example the limit gained an element in its covering spectrum due to longer and longer homotopies, it is also possible to gain an element in the covering spectrum without changing the topology of the space:

Example 5.4. We construct an example where an element of the covering spectrum appears in the limit. As in Example 2.20, let $M^{2}$ be the warped product manifold $\mathbb{R} \times_{f(r)} S^{1}$ where

$$
f(r)=2 \operatorname{Arctan}(-r)+\pi .
$$

Since $\lim _{r \rightarrow \infty} f(r)=0, \pi_{\text {slip }}(M)=\pi_{1}(M)$, and the covering spectrum is empty.

Now let $\left(X_{i}, x_{i}\right)=\left(M, p_{i}\right)$, where $r\left(p_{i}\right)=r_{i} \rightarrow-\infty$. Note that $\bar{B}_{x_{i}}(R)$ is then equipped with a warped product metric and

$$
f_{i}(r)=f\left(r-r_{i}\right)=2 \operatorname{Arctan}\left(-r+r_{i}\right)+\pi,
$$

which converges uniformly on $[-R, R]$ to

$$
f_{\infty}(r)=2 \pi
$$

Thus the pointed Gromov-Hausdorff limit is the standard cylinder whose covering spectrum is $\{\pi\}$. As above the cut-off covering spectra of these examples is empty, both for the $X_{i}$ and the limit space.

Next we construct an example where an element of the covering spectrum disappears in the limit without decreasing to 0 . This issue is not immediately solved by using the cut-off covering spectrum.

Example 5.5. Let $M^{2}$ be a cylinder with a small handle near a point $p$. Let $\left(X_{i}, x_{i}\right)=\left(M, p_{i}\right)$, where $d\left(p_{i}, p\right) \rightarrow \infty$. Then $\operatorname{CovSpec}\left(X_{i}\right)=\operatorname{Cov} \operatorname{Spec}(M)$ since the covering spectrum does not depend on the base point and the spectrum has its first element, $\lambda_{1}<\pi$, corresponding to the small handle. Yet for all $R>0$ just take $N_{R}$ large enough that

$$
d\left(p_{i}, p\right) \geq 2 R \quad \forall i \geq N_{R}
$$

Then $\bar{B}_{p_{i}}(R)$ are all isometric to balls of radius $R$ in a cylinder. So $\left(X_{i}, x_{i}\right)$ converge to a cylinder with a point. So the covering spectrum of the limit space does not include $\lambda_{1}$, and its only element is $\pi$. So we have locally compact $X_{i}$ converging to locally compact $X$ with

$$
\delta_{i} \in \operatorname{Cov} \operatorname{Spec}\left(X_{i}\right) \text { such that } \delta_{i}=\lambda_{1} \rightarrow \delta \notin \operatorname{Cov} \operatorname{Spec}(X) .
$$

In fact we have

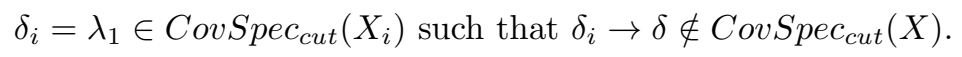


On the other hand, for any $R>0$ there exists $N_{R}>0$ such that

$$
\operatorname{CovSpec}_{\text {cut }}^{R}\left(X_{i}, x_{i}\right)=\{\pi\}
$$

for all $i \geq N_{R}$ because the handle is located outside $\bar{B}_{x_{i}}(R)$.

Finally we have the possibility that elements of the covering spectrum can grow to infinity. In this example we see that in essence a hole could expand until it snaps and is no longer a hole in the limit space:

Example 5.6. Let $\left(X_{r}, x_{r}\right)$ be formed where $X_{r}$ is a unit interval with $x_{r}$ on one end and a circle of circumference $2 \pi r$ attached to the other end, with a half line attached on the opposite side of the circle. Then $\operatorname{CovSpec}\left(X_{r}\right)=\{\pi r\}$.

Note that if one takes a sequence of $r_{i}$ diverging to infinity, $\left(X_{r_{i}}, x_{r_{i}}\right)$ converges in the pointed Gromov-Hausdorff sense to $\left(X_{\infty}, x_{\infty}\right)$, where $X_{\infty}$ is a unit interval attached to $x_{\infty}$ at one end and two half lines at the other end. So $X_{\infty}$ is simply connected and has an empty covering spectra.

This example is not simplified by using the cut-off covering spectra. In fact for any $R \geq 1, \operatorname{CovSpec}_{\text {cut }}^{R}\left(X_{r}\right)=\{\pi r\}$, and so $\operatorname{CovSpec}_{\text {cut }}\left(X_{r}\right)=\{\pi r\}$.

5.2. Convergence of the $R$ cut-off covering spectrum. In light of the above examples, it is natural to try to prove continuity of the $R$ cut-off covering spectra and then perhaps to apply this continuity to prove some form of continuity for the cut-off covering spectrum. Surprisingly, the statement of the continuity theorem for the cut-off spectrum is somewhat tricky:

Theorem 5.7. Let $\left(X_{i}, x_{i}\right)$ be complete locally compact length spaces converging in the pointed Gromov-Hausdorff sense to a locally compact space $(X, x)$. Bounded elements do not disappear: if we have a converging sequence

$$
\delta_{i} \in \operatorname{CovSpec}_{\text {cut }}^{R_{1}}\left(X_{i}, x_{i}\right) \text { and } \delta_{i} \rightarrow \delta>0, \text { then } \delta \in \operatorname{CovSpec}_{\text {cut }}^{R_{1}}(X, x) .
$$

Nor do elements suddenly appear: for any $R_{2}>R_{1}$ and if we have an element

$$
\delta \in \operatorname{CovSpec}_{\text {cut }}^{R_{1}}(X, x), \text { there are } \delta_{i} \in \operatorname{CovSpec} \operatorname{Sut}_{\text {cut }}^{R_{2}}\left(X_{i}, x_{i}\right) \text { such that } \delta_{i} \rightarrow \delta .
$$

Examples 5.2 and 5.6 demonstrate why one must assume $\delta_{i}$ converge in $(0, \infty)$. We now present examples demonstrating why we cannot take $R_{1}=R_{2}$ in (5.9).

Example 5.8. Let $\left(X_{r}, x_{r}\right)$ be formed by attaching a line segment of length $r$ to a circle of circumference $2 \pi$ and then continuing with a half line on the opposite side of the circle. The point $x_{r}$ will be the endpoint of the line segment not attached to the circle. If $r_{i} \rightarrow r_{\infty}$ it is easy to see that $\left(X_{r_{i}}, x_{r_{i}}\right)$ converges to $\left(X_{r_{\infty}}, x_{r_{\infty}}\right)$.

Note that $\operatorname{CovSpec}\left(X_{r}\right)=\{\pi\}$ and so does $\operatorname{CovSpec}_{\text {cut }}\left(X_{r}\right)$. On the other hand, $\operatorname{CovSpec}_{c u t}^{R}\left(X_{r}\right)=\emptyset$ when $r>R$, because then the circle is contained in $X_{r} \backslash \bar{B}_{x_{r}}(R)$. Otherwise $\operatorname{CovSpec} \operatorname{cut}_{\text {cut }}^{R}\left(X_{r}\right)=\{\pi\}$. Thus the sequence $X_{r_{j}}$ with $r_{j}$ decreasing to $R_{1}$ has

$$
\delta=\pi \in \operatorname{CovSpec} \operatorname{cut}_{\text {Rut }}^{R_{1}}\left(X_{r_{\infty}}\right),
$$

but $\operatorname{CovSpec} c_{\text {cut }}^{R_{1}}\left(X_{r_{i}}\right)=\emptyset$. However, taking $R_{2}>R_{1}$, eventually we have $r_{i}<R_{2}$, so we have

$$
\delta_{i}=\pi \in \operatorname{CovSpec} \operatorname{cut}_{2}^{R_{2}}\left(X_{r_{i}}\right) .
$$


The next example also illustrates the same phenomenon with a distinct cause:

Example 5.9. Let $M$ be a warped product manifold of the form $\mathbb{R} \times_{f} S^{1}$ where $f(t)=e^{-t^{2}}$. Fix $p$ in the level $t=0$.

Let $X_{r}=\bar{B}_{p}(r)$. So it is a closed ball, and if we wish to make it noncompact, we just attach a half line to it. We give it the induced length metric from $M$.

Let $r_{i}$ decrease to some $r_{\infty}>\pi$. Then $X_{i}=X_{r_{i}}$ converges to $X_{\infty}=X_{r_{\infty}}$.

Let $R_{1}=r_{\infty}$. The $R_{1}$-cut-off covering spectrum of $X_{\infty}$ includes $\delta$ equal to half the length of one of the components of the boundary of $B\left(p, R_{1}\right)$, because this curve is not homotopic to anything outside $\bar{B}_{p}\left(R_{1}\right)$. However the $R_{1}$-cutoff covering spectra of the $X_{i}$ are all empty because the loop is homotopic to a loop in $\partial B_{p}\left(r_{i}\right)$ which is outside $B\left(p, R_{1}\right)$. So once again we need $R_{2}>R_{1}$, and we need to wait for $r_{i}<R_{2}$ to get the cut-off covering spectra to converge.

One might also construct manifolds $M_{i}$ converging to $X_{\infty}$ by taking smoothed tubular neighborhoods of the $X_{i}$ in five dimensional Euclidean space.

Note that in the above examples, if one were to take $r_{j}$ increasing to $R_{0}$, the covering spectrum are all $\{\pi\}$. The difficulty arises because $r_{j}$ decreasing to $R_{\infty}$ are leaving the open set $\left(R_{\infty}, \infty\right)$ in the limit.

At first we thought we needed to take $R_{2}>R_{1}$ in (5.8) as well as (5.9), but due to the lack of examples proving this was necessary, we investigated further and discovered we could boost our proof of (5.8) using the local compactness of the limit space.

In order to prove this theorem we need to extend several results for covering spaces of compact spaces to $R$-cut-off spaces. The first is an adaption of Theorem 3.4 in SoWei1].

Proposition 5.10. Let $B\left(p_{i}, s_{i}\right) \subset B\left(p_{i}, S_{i}\right) \subset Y_{i}, i=1,2$, be balls, each with induced length metrics. Let $G\left(p_{1}, s_{1}, S_{1}, \delta_{1}\right)$ be the group of deck transformations of $\tilde{B}\left(p_{1}, S_{1}\right)_{\text {cut }}^{\delta_{1}, s_{1}}$.

If there is a pointed $\epsilon$-Hausdorff approximation $f: B\left(p_{1}, S_{1}\right) \rightarrow B\left(p_{2}, S_{2}\right)$, then for any $\delta_{1}>10 \epsilon$ and $\delta_{2}>\delta_{1}+10 \epsilon$ and $s_{2}<s_{1}-5 \epsilon$, there is a surjective homomorphism,

$$
\Phi: G\left(p_{1}, s_{1}, S_{1}, \delta_{1}\right) \rightarrow G\left(p_{2}, s_{2}, S_{2}, \delta_{2}\right)
$$

Proof of Proposition 5.10. We begin by describing a map for closed curves. For a closed curve $\gamma:[0,1] \rightarrow B\left(p_{1}, S_{1}\right)$ with $\gamma(0)=\gamma(1)=p_{1}$, construct a $5 \epsilon$-partition of $\gamma$ as follows. On $\Gamma:=\gamma([0,1])$ choose a partition $0=t_{0} \leq t_{1} \leq \cdots \leq t_{m}=1$ such that for $x_{i}=\gamma\left(t_{i}\right)$, one has $d\left(x_{i}, x_{i+1}\right)<5 \epsilon$ for $i=0, \ldots, m-1$. $\left\{x_{0}, \ldots, x_{m}\right\}$ is called a $5 \epsilon$-partition of $\gamma$.

Let $y_{m}=y_{0}=p_{2}$, and for each $x_{i}$ we set $y_{i}=f\left(x_{i}\right), i=1, \ldots, m-1$. Connect $y_{i}$ and $y_{i+1}$ by minimal geodesics in $B_{p_{2}}\left(S_{2}\right)$. This yields a closed curve $\bar{\gamma}$ in $B\left(p_{2}, S_{2}\right)$ based at $p_{2}$ consisting of $m$ minimizing segments, each having length $\leq 6 \epsilon$.

Any $\alpha \in G\left(p_{1}, s_{1}, S_{1}, \delta_{1}\right)$ can be represented by some rectifiable closed curve $\gamma$ in $B\left(p_{1}, S_{1}\right)$, so we can hope to define

$$
\Phi(\alpha)=\Phi([\gamma]):=[\bar{\gamma}] \in G\left(p_{2}, s_{2}, S_{2}, \delta_{2}\right) .
$$

First we need to verify that $\Phi$ doesn't depend on the choice of $\gamma$ such that $[\gamma]=\alpha$.

Using the fact that $18 \epsilon<\delta_{2}$ and loops which fit in balls of radius $\delta_{2}$ do not effect the representative of a class in $G\left(p_{2}, s_{2}, S_{2}, \delta_{2}\right)$, one can easily see that $[\bar{\gamma}]$ 
doesn't depend on the choice of minimizing curves $\bar{\gamma}_{i}$ or on the special partition $\left\{x_{1}, \ldots, x_{m}\right\}$ of $\gamma([0,1])$.

Moreover, additionally using the uniform continuity of a homotopy, one can similarly check that if $\gamma$ and $\gamma^{\prime}$ are homotopic in $B\left(p_{1}, S_{1}\right)$, then $[\bar{\gamma}]=\left[\bar{\gamma}^{\prime}\right]$ in $G\left(p_{2}, s_{1}-5 \epsilon, S_{2}, \delta_{2}\right)$. That is, we can take a homotopy $h:[0,1] \times[0,1] \rightarrow B\left(p_{1}, S_{1}\right)$, and we can take a grid on $[0,1] \times[0,1]$ small enough that homotopy maps the grid points to points $x_{i, j}$ that are less than $5 \epsilon$ apart from the images of their grid neighbors. Then we take $y_{i, j}=f\left(x_{i, j}\right)$ and connect neighbors according to the rules in the first paragraph. Finally we use the argument in the paragraph above this to see that the net created using the $y_{i, j}$ is a $\delta_{2}$ homotopy, so $[\bar{\gamma}]=\left[\bar{\gamma}^{\prime}\right]$ in $G\left(p_{2}, s_{2}-5 \epsilon, S_{2}, \delta_{2}\right)$. Thus we see that $\Phi$ is a homomorphism from $\pi_{1}\left(B\left(p_{1}, S_{1}\right), p_{1}\right)$ to $G\left(p_{2}, s_{2}, S_{2}, \delta_{2}\right)$. However $\alpha \in G\left(p_{1}, s_{1}, S_{1}, \delta_{1}\right)$, not $\pi_{1}\left(B\left(p_{1}, s_{1}\right), p_{1}\right)$.

Suppose $\gamma_{1}$ and $\gamma_{2}$ are both representatives of $\alpha \in G\left(p_{1}, s_{1}, S_{1}, \delta_{1}\right)$. Then $\gamma_{1} * \gamma_{2}^{-1}$ is, in $B\left(p_{1}, S_{1}\right)$, homotopic to a loop $\gamma_{3}$ generated by loops of the form $\alpha * \beta * \alpha^{-1}$, where $\beta$ is a closed path lying in a ball of radius $\delta_{1}$ or in $B\left(p_{1}, S_{1}\right) \backslash \bar{B}\left(p_{1}, s_{1}\right)$. So $\left[\overline{\gamma_{1}}\right]=\left[\overline{\gamma_{3}}\right] *\left[\overline{\gamma_{2}}\right]$ in $\pi_{1}\left(B\left(p_{1}, s_{1}\right), p_{1}\right)$. So we need only show that $\left[\overline{\gamma_{3}}\right]$ is trivial in $G\left(p_{2}, s_{2}, S_{2}, \delta_{2}\right)$.

In fact $\overline{\gamma_{3}}$ can be chosen as follows. The $y_{i}$ 's corresponding to the $x_{i}$ 's from the $\beta$ segments of $\gamma_{3}$ are all within $\delta_{1}+\epsilon$ of a common point, and the minimal geodesics between them are within $\delta_{1}+(1+6 / 2) \epsilon<\delta_{2}$. Furthermore, the $y_{i}$ 's corresponding to the $x_{i}$ 's from the $\alpha$ and $\alpha^{-1}$ segments of the curve can be chosen to correspond. Thus $\overline{\gamma_{3}}$ is generated by loops of the form $\alpha * \beta * \alpha^{-1}$ lying in $B\left(p_{2}, S_{2}\right)$, where $\beta$ is a closed path lying in a ball of radius $\delta_{2}$ or $B\left(p_{1}, S_{2}\right) \backslash B\left(p_{1}, s_{1}-5 \epsilon\right)$ and $\alpha$ is a path from $p_{2}$ to $\beta(0)$. So it is trivial.

Lastly, we need to show that $\Phi$ is onto. If $\bar{\alpha} \in G\left(p_{2}, s_{2}, S_{2}, \delta_{2}\right)$, it can be represented by some rectifiable closed curve $\sigma$ in $B\left(p_{2}, S_{2}\right)$ based at $p_{2}$. Choose an $\epsilon$-partition $\left\{y_{0}, \ldots, y_{m}\right\}$ of $\sigma$. Since $f: B\left(p_{1}, S_{1}\right) \rightarrow B\left(p_{2}, S_{2}\right)$ is an $\epsilon$-Hausdorff approximation, there are $x_{i} \in B\left(p_{1}, s_{1}\right), y_{i}^{\prime}=f\left(x_{i}\right) \in B\left(p_{2}, S_{2}\right)$, where $y_{0}^{\prime}=$ $y_{m}^{\prime}=p_{2}, x_{0}=x_{m}=p_{1}$ and $d_{B\left(p_{2}, S_{2}\right)}\left(y_{i}, y_{i}^{\prime}\right) \leq \epsilon$. Connect $y_{i}^{\prime}, y_{i+1}^{\prime}$ with a length minimizing curve in $B\left(p_{2}, S_{2}\right)$; this yields a piecewise length minimizing closed curve $\sigma^{\prime}$ in $B\left(p_{2}, S_{2}\right)$ based at $p_{2}$, where each segment has length $\leq 3 \epsilon$. So $\left[\sigma^{\prime}\right]=[\sigma]$ in $G\left(p_{2}, s_{1}-5 \epsilon, S_{2}, \delta_{2}\right)$. Now connect $x_{i}, x_{i+1}$ by length minimizing curves in $B\left(p_{1}, S_{1}\right)$; this yields a piecewise length minimizing $\gamma:[0,1] \rightarrow B\left(p_{1}, S_{1}\right)$ with base point $p_{1}$, where each segment has length $\leq 4 \epsilon$. So the curve $\gamma$ allows a $5 \epsilon$-partition and $[\gamma] \in G\left(p_{1}, s_{1}, S_{1}, \delta_{1}\right)$. By the construction, $\Phi([\gamma])=\bar{\alpha}$.

Therefore $\Phi$ is surjective.

Proposition 5.11. If a sequence of complete locally compact length spaces $X_{i}$ converges to a length space $X$ in the Gromov-Hausdorff topology, then for any $\delta>0, R>0, r>3 R$ there is a subsequence of $X_{i}$ and a sequence $r_{i} \rightarrow r$ such that $\tilde{B}\left(x_{i}, r_{i}\right)_{\text {cut }}^{\delta, R}$ also converges in the pointed Gromov-Hausdorff topology. Moreover, the limit space $B(x, r)_{\text {cut }}^{\delta, R}$ is a covering space of $B(x, r)$ satisfying

$$
\tilde{B}(x, r)_{\text {cut }}^{\delta, R} \rightarrow B(x, r)_{\text {cut }}^{\delta, R} \rightarrow \tilde{B}(x, r)_{\text {cut }}^{\delta^{\prime}, R^{\prime}}
$$

for all $0<R^{\prime}<R$ and $\delta^{\prime}>\delta$.

Proof. By the Appendix of SoWei2 we know that for a sequence $r_{i}$ converging to $r, B\left(x_{i}, r_{i}\right)$ converge with the induced length metric to $B(x, r)$. 
By [SoWei3, Proposition 7.3] and the fact that the closed balls $B\left(x_{i}, r_{i}\right)$ are compact sets, we know that $\tilde{B}\left(x_{i}, r_{i}\right)^{\delta}$ have a converging subsequence. So by Gromov's compactness theorem, they have a uniform bound $N(a, b)$, the number of disjoint balls of radius $a$ in a ball of radius $b$. By Proposition 4.8, $\tilde{B}\left(x_{i}, r_{i}\right)_{\text {cut }}^{\delta}$ covers $\tilde{B}\left(x_{i}, r_{i}\right)_{\text {cut }}^{\delta, R}$, so $N(a, b)$ can be used to count balls in $\tilde{B}\left(x_{i}, r_{i}\right)_{\text {cut }}^{\delta, R}$ as well. So by Gromov's compactness theorem, a subsequence of these spaces converges, and we will denote the limit space by $B(x, r)_{\text {cut }}^{\delta, R}$.

To complete the proof we adapt Theorem 3.6 of SoWei1. The fact that $\tilde{B}\left(x_{i}, r_{i}\right)_{\text {cut }}^{\delta, R}$ has isometries on balls of radius $\delta$ and outside $\bar{B}_{p}(R)$ guarantees that the limit does as well, so $B(x, r)_{\text {cut }}^{\delta, R}$ is a covering space for $B(x, r)$.

The isometries also guarantee it is covered by $\tilde{B}(x, r)_{\text {cut }}^{\delta, R}$. This can be seen by using the Unique Lifting Theorem (cf. Ma Lemma 3.1, page 123) and noting that if $C$ is a closed curve in $B(x, r)$ whose lift to $\tilde{B}(x, r)_{c u t}^{\delta, R}$ is closed, then it is homotopic to a curve which is created from curves of the form $\alpha \cdot \beta \cdot \alpha^{-1}$, where the $\beta$ are either in a ball of radius $\delta$ or outside $\bar{B}_{x}(R)$. So its lift to $B(x, r)_{\text {cut }}^{\delta, R}$ is also closed since $\pi^{\delta}$ is an isometry on $\delta$-balls and an isometry outside $\bar{B}_{x}(R)$. Therefore $\tilde{B}(x, r)_{\text {cut }}^{\delta, R}$ covers $B(x, r)^{\delta, R}$

To complete the proof we apply the Unique Lifting Theorem by contradiction. We assume there are $\delta^{\prime}>\delta$ and $R^{\prime}<R$ and that $C$ is a curve which lifts closed to $B(x, r)_{\text {cut }}^{\delta, R}$ but lifts open to $\tilde{B}(x, r)_{\text {cut }}^{\delta^{\prime}, R^{\prime}}$. Since this lift of $C$ is not closed, $[C] \in$ $G\left(x, r, R^{\prime}, \delta^{\prime}\right)$ is nontrivial.

Let $\epsilon>0$ be chosen sufficiently small such that

$$
\epsilon<\min \left\{\delta / 10,\left(\delta-\delta^{\prime}\right) / 10,\left(R-R^{\prime}\right) / 5\right\} .
$$

Take $i$ sufficiently large that we have an $\epsilon$-Hausdorff approximation $f_{i}: B\left(x_{i}, r_{i}\right) \rightarrow$ $B(x, r)$. Applying Proposition 5.10, we know there are surjective homomorphisms, $\Phi: G\left(x_{i}, r_{i}, R, \delta\right) \rightarrow G\left(x, r, R^{\prime}, \delta^{\prime}\right)$, so there are closed loops $C_{i} \in B\left(x_{i}, r_{i}\right)$ such that $\Phi\left(\left[C_{i}\right]\right)=[C]$.

By the construction of $\Phi, C_{i}$ can be chosen so these lifted curves $\tilde{C}_{i}$ converge to the lift of the limit of the curves, $\tilde{C}$ in $B(x, r)_{\text {cut }}^{\delta, R}$, and

$$
d_{B(x, r)_{c u t}^{\delta, R}}(\tilde{C}(0), \tilde{C}(1))=\lim _{i \rightarrow \infty} d\left(\tilde{C}_{i}(0), \tilde{C}_{i}(1)\right) .
$$

However the $\left[C_{i}\right]$ are nontrivial, so their lifts to $\tilde{B}\left(x_{i}, r_{i}\right)^{\delta, R}$ run between points $\tilde{C}_{i}(0) \neq \tilde{C}_{i}(1)$ satisfying

$$
d\left(\tilde{C}_{i}(0), \tilde{C}_{i}(1)\right) \geq \delta .
$$

Combining this with (5.15), we see that $\tilde{C}$ is not closed, and we have a contradiction.

At this point we could imitate the proof of Theorem 8.4 in [SoWei3] to prove Theorem 5.7 for $X_{i}$ which are compact balls. However, this would not help us prove Theorem 5.7 for noncompact spaces, as the cut-off covering spectrum of a ball does not match the cut-off covering spectrum of the space. Recall Examples 5.6 and 5.3 demonstrate that not only can holes become increasingly large, but homotopies may as well. One needs to control such phenomenon to complete the proof. 
Proof of Theorem [5.7. In order to prove the first statement (5.8) we first prove that given any $R_{2}>R_{1}$, if

$$
\delta_{i} \in \operatorname{CovSpec} \operatorname{cut}_{R_{1}}^{R_{1}}\left(X_{i}\right) \text { and } \delta_{i} \rightarrow \delta>0 \text {, then } \delta \in \operatorname{CovSpec}_{\text {cut }}^{R_{2}}(X) .
$$

Later we will boost this result to (5.8).

Assume

$$
\delta_{i} \in \operatorname{CovSpec}_{\text {cut }}^{R_{1}}\left(X_{i}\right)
$$

and $\delta_{i} \rightarrow \delta>0$. By Lemma 4.41, $\delta_{i} \in \operatorname{CovSpec}_{\text {cut }}^{R_{1}}\left(B\left(x_{i}, r\right)\right)$ for $r \geq 3\left(R_{1}+2 \delta_{i}\right)$. So $\tilde{B}\left(x_{i}, r\right)_{\text {cut }}^{\delta_{i}, R_{1}} \rightarrow \tilde{B}\left(x_{i}, r\right)_{\text {cut }}^{\delta^{\prime}, R_{1}}$ is nontrivial for all $\delta^{\prime}>\delta_{i}$. So for all $\delta^{\prime}>\delta>0$ and $\epsilon \in(0, \delta)$ we have $\delta-\epsilon<\delta_{i}<\delta^{\prime}$ for $i$ sufficiently large and

$$
\tilde{B}\left(x_{i}, r\right)_{\text {cut }}^{\delta-\epsilon, R_{1}} \rightarrow \tilde{B}\left(x_{i}, r\right)_{\text {cut }}^{\delta^{\prime}, R_{1}}
$$

is nontrivial. Now take the limit as $i \rightarrow \infty$, and we obtain that

$$
B(x, r)_{\text {cut }}^{\delta-\epsilon, R_{1}} \rightarrow B(x, r)_{\text {cut }}^{\delta^{\prime}, R_{1}}
$$

is nontrivial.

This is true for all $\epsilon \in(0, \delta)$ and $\delta^{\prime}>\delta$. By the properties of limit covers in Proposition 5.11 we have for all $\epsilon \in(0, \delta), \delta^{\prime \prime}>\delta^{\prime}$, and $R^{\prime} \in\left(R_{1}, R_{2}\right)$,

$$
\tilde{B}(x, r)_{\text {cut }}^{\delta-\epsilon, R_{1}} \rightarrow B(x, r)_{\text {cut }}^{\delta-\epsilon, R_{1}} \text { and } B(x, r)_{\text {cut }}^{\delta^{\prime}, R_{1}} \rightarrow \tilde{B}(x, r)_{\text {cut }}^{\delta^{\prime \prime}, R^{\prime}} .
$$

Therefore $\tilde{B}(x, r)_{\text {cut }}^{\delta-\epsilon, R_{1}} \rightarrow \tilde{B}(x, r)_{c u t}^{\delta^{\prime \prime}, R^{\prime}}$ is nontrivial.

By Proposition 4.45 we then know that since $B(x, r)$ is compact and $R_{2}>R^{\prime}$, we have

$$
\operatorname{CovSpec}_{\text {cut }}^{R_{2}}(B(x, r)) \cap\left[\delta-\epsilon, \delta^{\prime \prime}\right) \neq \emptyset .
$$

Taking $\epsilon$ to 0 and $\delta^{\prime \prime}$ to $\delta$, we get

$$
\delta \in \operatorname{CovSpec} \operatorname{cut}_{\text {cut }}^{R_{2}}(B(x, r)) .
$$

This is true for all sufficiently large $r$, so by Lemma 4.40

$$
\delta \in \operatorname{CovSpec}_{\text {cut }}^{R_{2}}(X),
$$

which completes proof of (5.17).

We now boost the statement (5.17) to prove (5.8). Again fix $R_{1}>0$. Suppose

$$
\delta_{i} \in \operatorname{CovSpec}_{c u t}^{R_{1}}\left(X_{i}\right)
$$

and $\delta_{i} \rightarrow \delta>0$. Let $X$ be the Gromov-Hausdorff limit of the $X_{i}$.

Proposition 4.45 says that for $R_{2}=R_{i}$ sufficiently close to $R_{1}$,

$$
\tilde{X}_{\text {cut }}^{\delta, R_{2}}=\tilde{X}_{\text {cut }}^{\delta, R_{1}}
$$

putting this together with Proposition 4.44 says

$$
\delta \notin \operatorname{CovSpec}_{\text {cut }}^{R_{2}}(X) \backslash \operatorname{CovSpec}_{\text {cut }}^{R_{1}}(X) .
$$

We apply (5.17) to say

$$
\delta \in \operatorname{CovSpec}_{\text {cut }}^{R_{2}}(X)
$$

But then

$$
\delta \in \operatorname{CovSpec}_{\text {cut }}^{R_{1}}(X),
$$

which gives us (5.8). 
Now we prove the second statement (5.9), given

$$
\delta \in \operatorname{CovSpec}_{\text {cut }}^{R_{1}}(X)
$$

and any $R_{2}>R_{1}$, show there exists

$$
\delta_{i} \in \operatorname{CovSpec} R_{c u t}^{R_{2}}\left(X_{i}\right)
$$

such that $\delta_{i} \rightarrow \delta$.

We assume on the contrary that there is a gap:

$$
\exists \epsilon>0 \text { such that } \operatorname{CovSpec} R_{\text {cut }}^{R_{2}}\left(X_{i}\right) \cap(\delta-2 \epsilon, \delta+2 \epsilon)=\emptyset .
$$

By Lemma 4.40, for $r \geq 3\left(R_{2}+2 \delta+4 \epsilon\right)$,

$$
\operatorname{CovSpec}_{\text {cut }}^{R_{2}}\left(B\left(x_{i}, r\right)\right) \cap(\delta-2 \epsilon, \delta+2 \epsilon)=\emptyset .
$$

By Lemma 4.14 we then have for any $R_{2}^{\prime} \leq R_{2}$,

$$
\operatorname{CovSpec}_{\text {cut }}^{R_{2}^{\prime}}\left(B\left(x_{i}, r\right)\right) \cap(\delta-2 \epsilon, \delta+2 \epsilon)=\emptyset .
$$

So the covering

$$
\tilde{B}\left(x_{i}, r\right)_{c u t}^{\delta-\epsilon, R_{2}^{\prime}} \rightarrow \tilde{B}\left(x_{i}, r\right)_{c u t}^{\delta+\epsilon, R_{2}^{\prime}}
$$

is trivial. By Proposition 5.11 we have a subsequence of the $i$ such that

$$
\tilde{B}\left(x_{i}, r\right)_{c u t}^{\delta-\epsilon, R_{2}^{\prime}} \rightarrow B(x, r)_{c u t}^{\delta-\epsilon, R_{2}^{\prime}}
$$

and

$$
\tilde{B}\left(x_{i}, r\right)_{c u t}^{\delta+\epsilon, R_{2}^{\prime}} \rightarrow B(x, r)_{c u t}^{\delta+\epsilon, R_{2}^{\prime}}
$$

since the sequence of the covering map is trivial, the covering limit map

$$
B(x, r)_{c u t}^{\delta-\epsilon, R_{2}^{\prime}} \rightarrow B(x, r)_{c u t}^{\delta+\epsilon, R_{2}^{\prime}}
$$

is also trivial.

By Proposition 5.11, for any $R_{2} \geq R_{2}^{\prime}>R_{1}$,

$$
B(x, r)_{\text {cut }}^{\delta-\epsilon, R_{2}^{\prime}} \rightarrow \tilde{B}(x, r)_{\text {cut }}^{\delta, R_{1}} \rightarrow \tilde{B}(x, r)_{\text {cut }}^{\delta+\epsilon, R_{1}} \rightarrow B(x, r)_{\text {cut }}^{\delta+\epsilon, R_{1}} .
$$

By Proposition 4.45 taking $R_{2}^{\prime}>R_{1}$ sufficiently close to $R_{1}$, the covering

$$
\tilde{B}(x, r)_{c u t}^{\delta-\epsilon, R_{2}^{\prime}} \rightarrow \tilde{B}(x, r)_{c u t}^{\delta+2 \epsilon, R_{1}}
$$

is trivial. Using Proposition [5.11, the covering

$$
B(x, r)_{\text {cut }}^{\delta-\epsilon, R_{2}^{\prime}} \rightarrow B(x, r)_{c u t}^{\delta+\epsilon, R_{1}}
$$

is trivial.

Apply this to (5.39) and we get trivial covers in (5.39). Therefore $\delta \notin$ CovSpec cut $R_{\text {cut }}^{R_{1}}(B(x, r))$.

By Lemma 4.41, $\delta \notin \operatorname{CovSpec}_{c u t}^{R_{1}}(X)$. That is a contradiction. 
5.3. Convergence of the cut-off covering spectrum. Theorem 5.7 combined with Proposition 4.17 gives the following result that elements in the cut-off covering spectrum do not suddenly appear in limits. Example 5.5 demonstrates that elements of the cut-off covering spectrum can disappear in the limit by sliding out to infinity. Unlike the $R$ cut-off covering spectrum, all handles are now visible.

Theorem 5.12. Let $\left(X_{i}, x_{i}\right)$ be complete locally compact length spaces converging in the pointed Gromov-Hausdorff sense to a locally compact space $(X, x)$. Then

$$
\text { for any } \delta \in \operatorname{CovSpec}_{\text {cut }}(X) \text {, there is } \delta_{i} \in \operatorname{CovSpec}_{\text {cut }}\left(X_{i}\right)
$$

such that $\delta_{i} \rightarrow \delta$.

This provides an immediate application:

Corollary 5.13. If $X_{i}$ are simply connected locally compact length spaces converging in the pointed Gromov-Hausdorff sense to a locally compact space $(X, x)$, then $\operatorname{CovSpec}_{\text {cut }}(X)=\emptyset$.

Proof of Theorem 5.12. If $\delta \in \operatorname{CovSpec}_{\mathrm{cut}}(X)$, by Proposition 4.17,

$$
\delta \in C l_{\text {lower }} \bigcup_{R>0} \operatorname{Cov} \operatorname{Spec}_{\text {cut }}^{R}(X) .
$$

So there are $R_{k}$ increasing to infinity and

$$
\delta_{k} \in \operatorname{CovSpec}_{\text {cut }}^{R_{k}}(X)
$$

such that $\delta_{k} \rightarrow \delta$. By Theorem 5.7 and $R_{k+1}>R_{k}$, for each $\delta_{k}$ we have

$$
\delta_{k}^{i} \in \operatorname{CovSpec}_{\text {cut }}^{R_{k+1}}\left(X_{i}\right) \subset \operatorname{CovSpec} \operatorname{cut}_{\text {cut }}\left(X_{i}\right)
$$

such that $\delta_{k}^{i} \rightarrow \delta_{k}$.

By a diagonal process, we have

$$
\delta_{i}=\delta_{k_{i}}^{i} \in \operatorname{CovSpec} \operatorname{cut}_{\text {cut }}\left(X_{i}\right)
$$

such that $\delta_{i} \rightarrow \delta$.

Question 5.14. Is local compactness a necessary condition in our convergence theorems [Theorem 5.7 and Theorem 5.12]? This condition is used in a few crucial steps of the proof. It is used in Lemma 3.9 to apply the pigeon hole principle to control the lengths of shortest representative curves. It is also used in Proposition 5.11 to prove that the delta covers of balls converge in the Gromov-Hausdorff sense when these balls converge in the Gromov-Hausdorff sense. This proposition is based on a result in [SoWei3], which requires compactness. Proposition 4.45, which requires compactness, is applied to balls in the proof. Finding examples demonstrating the necessity of local compactness or compactness in any of these results would be of interest.

Recall that the pulled ribbon construction introduced in Section 4.6 was used to obtain important examples which are not locally compact. Here, however, the ribbon construction does not immediately help:

Remark 5.15. Suppose one were to attach pulled ribbons to the sequence of manifolds in Example 5.4 in an attempt to prove that local compactness is necessary in Theorem 5.12. The difficulty is that as soon as the spaces are bounded, there is no way to effectively use a base point to differentiate the spaces from one another. 
Thus the sequence is just a repeating space and converges to itself. It does not produce a counterexample. The same effect happens if we try to attach pulled ribbons to capped cylinders which converge to cylinders in the pointed Gromov-Hausdorff sense (see Example 5.3).

If we choose $M_{j}$ to be warped products with two cusps that are isometric to cylinders on $[-j, j]$, such spaces would converge in the pointed Gromov-Hausdorff sense to a cylinder. However, when we attach pulled ribbons to them, the sequence does not even converge: each space is a definite Gromov-Hausdorff distance apart from each other. The only reason the $M_{j}$ converged was because the pointed GH convergence only saw the center cylindrical region, but when the whole space is bounded, the whole space needs to behave in a uniform way.

5.4. Applications of convergence. In this section we observe the following topological consequence of our convergence results:

Theorem 5.16. If $X_{i}$ are complete locally compact length spaces that satisfy the loops to infinity property and converge in the pointed Gromov-Hausdorff sense to a locally compact semi-locally simply connected limit space $X$, then either $X$ has at least two ends or $X$ has the loops to infinity property.

Proof. By Theorem 4.23, CovSpec cut $\left(X_{i}\right)$ are trivial. So, applying Theorem 5.12, we see $\operatorname{CovSpec} \operatorname{cut}_{\text {cut }}(X)$ must be trivial. To complete the proof we just apply Theorem 4.24

One can think of this theorem as the complete version of the theorem in SoWei1 which says that compact Gromov-Hausdorff limits of simply connected compact manifolds are simply connected.

Example 5.17. The SoWei1 theorem is not true for noncompact limits with pointed Gromov-Hausdorff convergence, as can be seen by taking sequences of ellipsoids $M_{j}^{2}$ which stretch out to a cylinder $S^{1} \times \mathbb{R}$ or $M_{j}^{2} \times \mathbb{R}$ converging to $S^{1} \times \mathbb{R}^{2}$. Thanks to our new theorem we see that while holes may form in a limit, they cannot be handles.

Example 5.18. Notice that if one takes a disk and stretches two points out to infinity, then the limit is a disk with two cusps, which is no longer simply connected. Nor does it have the loops to infinity property. This is because a loop wrapping once around each cusp is not homotopic to loops approaching infinity. However the fundamental group of the space is generated by elements with loops to infinity property.

Example 5.19. Note that one can have compact $M_{j}$ with $\pi_{2}\left(M_{j}\right)$ nontrivial converging to a space with nontrivial $\pi_{2}$. This can be seen by taking $M_{j}$ diffeomorphic to the plane with warped product metrics

$$
d r^{2}+f_{j}^{2}(r) d \theta^{2}
$$

where $f(r)=r\left((1-r)^{2}+(1 / k)\right)$, so that the Gromov-Hausdorff limit as $k \rightarrow \infty$ is homoemorphic to a sphere attached to a plane. So we cannot hope to control higher homotopy, although an investigation of [ShSo1] reveals a close relationship between the loops to infinity property and the codimension one integer homology of the space. 
5.5. Tangent cones at infinity. A complete noncompact space, $X$, is said to have a tangent cone at infinity if the Gromov-Hausdorff limit of a sequence of inward rescalings $\left(X / r_{j}, x\right)$ with $r_{j} \rightarrow \infty$ has a limit in the pointed Gromov-Hausdorff sense. While this limit space is called a cone, it is not a metric cone except in very special situations, such as when $X$ has nonnegative sectional curvature [BBI]. In fact the tangent cone at infinity of a manifold need not even be simply connected, as can be seen in this well-known example:

Example 5.20. Let $M^{2}$ be created by taking a cone, smoothing off the tip and adding handles, $r_{i} H$, at a distance $r_{i}$ from the old tip. We write $r_{i} H$ because we are rescaling the handle $H$ by $r_{i}$, so that the handles are growing. Then $M^{2} / r_{i}$ converges to a cone with a handle attached at a distance 1 from the tip. If $\lim r_{i+1} / r_{i}=\infty$, then the tangent cone has only one handle, but if $\lim r_{i+1} / r_{i}=d$, then the tangent cone has infinitely many handles located at $\left\{d^{j}: j \in \mathbb{Z}\right\}$, so the tangent cone at infinity, $Y$, has locally infinite topological type at its tip. Furthermore, $Y$ has no universal cover and $\operatorname{CovSpec}(Y)=\operatorname{CovSpec}_{c u t}(Y)$ have infinitely many elements.

Remark 5.21. Menguy has created similar examples demonstrating that the tangent cone at infinity of a manifold with nonnegative Ricci curvature can have locally infinite topological type, although his examples are simply connected because his handles are higher dimensional (cf. [ShSo2]). In [SoWei3] we proved the tangent cones at infinity of manifolds with Ricci $\geq 0$ have universal covers.

Using our results we can prove

Theorem 5.22. If $X$ is a complete locally compact length space and CovSpec cut $(X)$ is bounded, then any tangent cone at infinity for $X$ has a trivial cut-off covering spectrum.

First note that the following lemma holds.

Lemma 5.23. If we rescale a metric space $X$ to get a new metric space $X / r$, then the elements of the covering spectrum and cut-off covering spectrum scale proportional to the distance:

$$
\operatorname{CovSpec}(X / r)=\operatorname{CovSpec}(X) / r
$$

and

$$
\operatorname{CovSpec}_{\text {cut }}(X / r)=\operatorname{CovSpec}_{\text {cut }}(X) / r .
$$

Furthermore, we have

$$
\operatorname{CovSpec}_{\text {cut }}^{R / r}(X / r)=\operatorname{CovSpec}_{\text {cut }}^{R}(X) / r .
$$

This lemma follows immediately from the definitions.

Proof of Theorem 5.22, If we rescale a space dividing the metric by $r$, then by Lemma 5.23 we have

$$
\operatorname{CovSpec}_{\text {cut }}^{R / r}(X / r)=\operatorname{CovSpec}_{\text {cut }}^{R}(X) / r .
$$

So applying Proposition 4.17 we have

$$
\operatorname{CovSpec}_{\text {cut }}(X / r)=\operatorname{CovSpec}_{\text {cut }}(X / r) \subset[0, \operatorname{Max}(\operatorname{CovSpec}(X)) / r] .
$$

Any tangent cone at infinity, $Y$, is the Gromov-Hausdorff limit of $X / r_{i}$ with $r_{i} \rightarrow \infty$, so by Theorem [5.12, CovSpec cut $(Y) \subset\{0\}$ and is, thus, trivial. 
This proposition implies that the tangent cones at infinity of manifolds with bounded covering spectra have trivial cut-off covering spectra. However, they need not have trivial covering spectra, even when the manifold has a trivial covering spectrum:

Example 5.24. Let $M$ be the length space constructed by attaching a sequence of widening cylinders to a plane as follows. Take a flat Euclidean plane and remove disks of radius $2^{j} / 4$ about the points $\left(2^{j}, 0\right)$, where $j=1,2,3 \cdots$. Now attach standard cylinders of radius $2^{j} / 4$ and length $4^{j}$ to each edge. Then attach the removed disks back on the far side of the cylinders. This creates a simply connected space with a trivial, and thus bounded, covering spectrum.

If we rescale $M$ by $1 / 2^{j}$ we get a tangent cone at infinity which is not even semilocally simply connected. It is a plane with disks of radius $2^{j} / 4$ centered at $\left(2^{j}, 0\right)$ removed and with half cylinders attached for all values of $j \in \mathbb{Z}$. Its cut-off covering spectrum is clearly still trivial, but its covering spectrum is very large.

Note that, without much difficulty, we could smooth $M$ to make it a manifold and still get the same tangent cone at infinity.

\section{Applications with Curvature bounds}

In this section we describe applications to complete noncompact Riemannian manifolds with lower bounds on their sectional and Ricci curvature and on their limit spaces.

6.1. Sectional curvature and the soul theorem. Cheeger-Gromoll ChGr proved that complete manifolds with nonnegative sectional curvature are diffeomorphic to normal bundles over totally geodesic compact submanifolds called souls. Sharafutdinov $[\mathrm{Sh}]$ then proved there was a distance nonincreasing retraction to the soul: $P: M \rightarrow S$. Perelman [Pe1, $\mathrm{Pe} 2$ showed that $P$ is a Riemannian submersion and extended the distance nonincreasing retraction to complete Alexandrov spaces with nonnegative curvature. Using the distance nonincreasing retraction we can show that the covering spectrum of these spaces behave exactly like the covering spectrum of a compact space.

Theorem 6.1. If $M^{n}$ is a complete noncompact Alexandrov space with nonnegative curvature, then

$$
\operatorname{CovSpec}\left(M^{n}\right)=\operatorname{CovSpec}\left(S^{k}\right)=\operatorname{CovSpec}\left(\bar{T}_{R}\left(S^{k}\right)\right),
$$

where $S^{k}$ is a soul and $\bar{T}_{R}\left(S^{k}\right)$ is the closed tubular neighborhood of radius $R$ around $S^{k}$.

In light of the above paragraph Theorem 6.1 follows directly from the following theorem, which we also use at the end of the paper.

Theorem 6.2. If $M$ has a totally geodesic soul $S^{k}$ with a distance nonincreasing retraction $P: M \rightarrow S^{k}$, then

$$
\operatorname{Cov} \operatorname{Spec}\left(M^{n}\right)=\operatorname{Cov} \operatorname{Spec}\left(S^{k}\right)=\operatorname{Cov} \operatorname{Spec}\left(\bar{T}_{R}\left(S^{k}\right)\right) .
$$

Note that this is significantly stronger than the loops to infinity property, which says that curves are homotopic outward. In fact the curves are homotopic inward to curves in the soul. 
Proof. If $\delta \in \operatorname{CovSpec}\left(M^{n}\right)$, then $\tilde{M}^{\delta^{\prime}} \neq \tilde{M}^{\delta}$ for all $\delta^{\prime}>\delta$. Namely $\pi(M, \delta) \neq$ $\pi\left(M, \delta^{\prime}\right)$ for all $\delta^{\prime}>\delta$. So for each $\delta^{\prime}>\delta$, there is $\gamma_{\delta^{\prime}}$ in $\pi_{1}(M)$ such that $g_{\delta^{\prime}}$ is generated by elements lying $\delta^{\prime}$-balls of $M$ but not generated by elements lying $\delta$-balls of $M$. Since $P: M^{n} \rightarrow S^{k}$ is distance nonincreasing, $P$ maps balls of $M$ to the same or smaller size ball of $S^{k}$. Hence $P\left(\gamma_{\delta^{\prime}}\right)$ is generated by elements lying $\delta^{\prime}$-balls of $S^{k}$. Since $P$ is a retraction $P\left(\gamma_{\delta^{\prime}}\right)$ is freely homotopic to $\gamma_{\delta^{\prime}}$, so it cannot be generated by elements lying $\delta$-balls of $M$, and therefore not $\delta$-balls of $S^{k}$. Now for each $\delta^{\prime}>\delta$, we have that $P\left(\gamma_{\delta^{\prime}}\right)$ is generated by elements lying $\delta^{\prime}$-balls of $S^{k}$ but not $\delta$-balls of $S^{k}$. This means $\delta \in \operatorname{CovSpec}\left(S^{k}\right)$.

Conversely, if $\delta \in \operatorname{CovSpec}\left(S^{k}\right)$, for each $\delta^{\prime}>\delta$ there is $\gamma_{\delta^{\prime}}$ in $\pi_{1}(S)$ such that $g_{\delta^{\prime}}$ is generated by elements lying $\delta^{\prime}$-balls of $S$ but not generated by elements lying $\delta$-balls of $S^{k} . g_{\delta^{\prime}}$ is not generated by elements lying $\delta$-balls of $M^{n}$ either, by the above argument. Therefore $\delta \in \operatorname{CovSpec}\left(M^{n}\right)$.

Since $\pi_{1}\left(M^{n}\right)=\pi_{1}\left(S^{k}\right)=\pi_{1}\left(\bar{T}_{R}\left(S^{k}\right)\right)$, we have (6.3) $\operatorname{CovSpec}\left(\bar{T}_{R}\left(S^{k}\right)\right) \subset \operatorname{CovSpec}\left(S^{k}\right)$ and $\operatorname{CovSpec}\left(M^{n}\right) \subset \operatorname{CovSpec}\left(\bar{T}_{R}\left(S^{k}\right)\right)$.

Hence they are all equal.

6.2. An almost soul theorem. In this section we apply our results to complete noncompact Riemannian manifolds with nonnegative sectional curvature. To do so we first study sequences of manifolds with sect $\geq-\epsilon_{i}$, where $\epsilon_{i}$ converges to 0 , and then prove an almost soul theorem:

Theorem 6.3. If $\left(X_{i}, x_{i}\right)$ are complete locally compact length spaces converging in the pointed Gromov-Hausdorff sense to a locally compact length space $(Y, y)$ such that $Y$ is the normal bundle over a totally geodesic soul with a distance nonincreasing retraction $P: Y \rightarrow S$, then there exist compact almost-souls $S_{i} \subset X_{i}$ with

$$
\operatorname{diam}\left(S_{i}\right)=D_{i} \rightarrow \operatorname{diam}(S) \text { and } d_{X_{i}}\left(x_{i}, S_{i}\right) \rightarrow d_{Y}(y, S)
$$

such that for any $b>a>0$ and any $R_{2}>R_{1}>0$ we have

$$
d_{H}\left(\operatorname{CovSpec}\left(\bar{T}_{R_{1}}\left(S_{i}\right)\right) \cap[a, b], \operatorname{CovSpec}\left(\bar{T}_{R_{2}}\left(S_{i}\right)\right) \cap[a, b]\right) \rightarrow 0,
$$

where $\bar{T}_{r}(A)$ denotes the closed tubular neighborhood about $A$ with the induced length metric.

Note that the almost souls constructed here are not totally geodesic but are compact. They are only soul-like in the sense that loops slide toward them, so that the covering spectrum is the same on two distinct tubular neighborhoods.

Proof. Note that $\bar{T}_{R_{2}}(S)$ is contained in some large ball $B_{R_{3}}(y)$ and that there must be an $\epsilon_{i}$ almost isometry $f_{i}: B_{x_{i}}\left(R_{i}\right) \rightarrow B_{y}\left(R_{3}\right)$. Let $S_{i}$ be the closure of the preimage of the soul $S \subset Y$ :

$$
S_{i}=C l\left(f_{i}^{-1}(S)\right) .
$$

Suppose the theorem is false. Then there exists $b>a>0$ and $R_{2}>R_{1}>0$ and $\delta_{i} \in[a, b]$ such that

(6.7)

$\delta_{i} \in \operatorname{CovSpec}\left(\bar{T}_{R_{1}}\left(S_{i}\right)\right) \backslash \operatorname{CovSpec}\left(\bar{T}_{R_{2}}\left(S_{i}\right)\right) \cup \operatorname{Cov} \operatorname{Spec}\left(\bar{T}_{R_{1}}\left(S_{i}\right)\right) \backslash \operatorname{Cov} \operatorname{Spec}\left(\bar{T}_{R_{2}}\left(S_{i}\right)\right)$.

Since $\delta_{i} \in[a, b]$, a subsequence converges to some $\delta \in[a, b]$. 
By applying Theorem 8.4 of SoWei3 to $\bar{T}_{R_{1}}\left(S_{i}\right)$ and $\bar{T}_{R_{2}}\left(S_{i}\right)$, which converge to $\bar{T}_{R_{1}}(S)$ and $\bar{T}_{R_{2}}(S)$, we know that

(6.8)

$\delta \in \operatorname{CovSpec}\left(\bar{T}_{R_{1}}(S)\right) \backslash \operatorname{CovSpec}\left(\bar{T}_{R_{2}}(S)\right) \cup \operatorname{CovSpec}\left(\bar{T}_{R_{1}}(S)\right) \backslash \operatorname{CovSpec}\left(\bar{T}_{R_{2}}(S)\right)$.

However no such $\delta$ exists by Theorem 6.2 .

The following corollary follows immediately from Theorem 6.3 and Theorem 6.1 .

Corollary 6.4. Given any $h>0$, any $b>a>0$ and any $R_{2}>R_{1}>0$ there exists $\epsilon=\epsilon\left(h, a, b, R_{1}, R_{2}\right)>0$ sufficiently small such that if $M^{n}$ has sect $\geq-\epsilon$, then there is a compact $S \subset M^{n}$ such that the Hausdorff distance

$$
d_{H}\left(\operatorname{CovSpec}\left(\bar{T}_{R_{1}}(S)\right) \cap[a, b], \operatorname{CovSpec}\left(\bar{T}_{R_{2}}(S)\right) \cap[a, b]\right)<h .
$$

Rescaling this corollary and consulting Theorem 6.3 to locate the almost souls, we get:

Corollary 6.5. Given any $h, r, D>0$, any $b>a>0$ and any $R_{2}>R_{1}>0$ there exists $\epsilon=\epsilon\left(h, a, b, R_{1}, R_{2}\right)>0$ sufficiently small such that if $M^{n}$ has sect $\geq-1$ and $p \in M^{n}$, then there is a compact $S \subset M^{n}$ with $\operatorname{diam}(S) \leq D$ and $d(S, p)<r$ such that

$$
d_{H}\left(\operatorname{CovSpec}\left(\bar{T}_{R_{1} \epsilon}(S)\right) \cap[a \epsilon, b \epsilon], \operatorname{Cov} \operatorname{Spec}\left(\bar{T}_{R_{2} \epsilon}(S)\right) \cap[a \epsilon, b \epsilon]\right)<h \epsilon .
$$

We can call such sets $S$ satisfying (6.10) subscaled souls and call manifolds with this property manifolds with many subscaled souls.

Note that a single space with thinner and thinner cylindrical subsets would satisfy this corollary, but a space with tiny handles would not. A hyperbolic manifold will not have arbitrarily small handles, but will rather either look locally thick like Euclidean space, or thin like in a cusp where it is somewhat cylindrical. Intuitively, this corollary is saying manifolds with a uniform lower bound on sectional curvature have a similar behavior.

6.3. Nonnegative Ricci curvature. When a complete noncompact manifold has nonnegative Ricci curvature, it doesn't always have a soul. However, the first author proved that such a manifold either has the loops to infinity property [Definition 4.22 ] or it is the flat normal bundle over a compact totally geodesic soul So, Theorem 11]. An example where the latter occurs is the infinite Moebius strip. Notice that the double cover of the infinite Moebius strip is a flat cylinder. In fact Theorem 11 of [So] states that a double cover always splits isometrically when the space fails to have the loops to infinity property. This means that it is the isometric product of a line with another metric space.

This has profound implications on the cut-off covering spectrum:

Theorem 6.6. Let $X$ be a complete noncompact manifold with Ricci $\geq 0$. If CovSpec cut $_{(X)}$ is not empty, then $X$ has a double cover which splits isometrically and $X$ is a flat normal bundle over a compact totally geodesic soul, in which case $X$ has one element in the covering spectrum and is half the length of the shortest closed geodesic which lifts as an open curve to this double cover.

Corollary 6.7. If $M$ is a Riemannian manifold with Ricci curvature strictly positive at one point and Ricci $\geq 0$ everywhere, then $\operatorname{CovSpec}_{\text {cut }}(M)=\emptyset$. 
Proof of Theorem [6.6. In [So] it is proven that a complete noncompact manifold, $M^{m}$, with nonnegative Ricci curvature has the loops to infinity property unless a double cover splits isometrically and $M^{m}$ is the flat normal bundle over a compact totally geodesic soul [Theorem 7, Theorem 11].

When $M$ has the loops to infinity property, we just apply Theorem 4.23 ,

When $M$ has a soul $S$ and a split double cover, $\mathbb{R} \times K$, there is a collection of loops $C$ which lift open to this double cover. Each $C$ lifts to a curve of the form $(a, b)$ where $a$ is a loop in $\mathbb{R}$ and $b$ is a loop in $K$, the compact double cover of the soul. Note that any curve $C$ is freely homotopic to the projection $\bar{C}$ of the loop $(0, b)$ and $\bar{C}$ is shorter than $C$.

Let $\delta_{0}=\inf L(C) / 2=\inf L(\bar{C}) / 2$. Then $2 \delta_{0}$ is the length of the shortest open path in $K$ which projects to a loop in the soul. So it is positive and is achieved by a closed geodesic $\gamma$ which represents some element $g \in \pi_{1}(S, \gamma(0)) \subset \pi_{1}(M, \gamma(0))$.

We claim $\operatorname{CovSpec}_{\text {cut }}(X)=\left\{\delta_{0}\right\}$.

Given any closed curve $\sigma$ based at $\gamma(0)$, either $\sigma$ lifts to an open path or to a closed loop in the double cover. If $\sigma$ lifts as a closed loop to the split double cover, then its lift has the loops to infinity property, so we can project the homotopy down and see that $\sigma$ has the loops to infinity property and will not contribute to the cut-off covering spectrum.

So suppose $\sigma$ lifts as an open path to $\tilde{X}_{c u t}^{\delta, R}$ for all values of $\delta$ and $R$. Since the split double cover is a double cover, $\sigma$ must be homotopic to $\gamma$ following a loop which lifts to a closed loop to the double cover. Since all loops in the split double cover have the loops to infinity property, that loop must as well. So $\sigma$ must be $\delta, R$ homotopic to $\gamma$ for any value of $\delta$ and $R$.

Thus $\gamma$ alone suffices to detect the distinct $\tilde{X}_{c u t}^{\delta, R}$. So there is only one element in the $R$ cut-off covering spectrum for any value of $R$. This element must be $\delta_{0}$ because $\gamma$ is the shortest loop in the class that doesn't have the loops to infinity property.

6.4. Further directions. Here we discuss potential applications to manifolds with Ricci $\geq-(n-1)$. These applications will arise by studying limits of manifolds with Ricci $\geq-\epsilon_{i}$. These limits spaces are complete locally compact length spaces $\mathrm{Gr}$.

Let $X$ be the Gromov-Hausdorff limit of complete noncompact Riemannian manifolds $M_{i}$ with $\operatorname{Ricci}\left(M_{i}\right) \geq-\epsilon_{i} \rightarrow 0$. Such a length space is very similar to a Riemannian manifold with nonnegative Ricci curvature. Cheeger and Colding have proven that the splitting theorem holds on such a space ChCo1, and the authors have proven that such spaces have universal covers SoWei2. Combining these facts with the proof of Theorem 7 in $[\mathrm{So}$, the authors proved that $X$, either has the loops to infinity property or the universal cover splits isometrically [SoWei2, Cor. 4.9].

Thus, using the proof of Theorem 6.6. we can conclude the following:

Theorem 6.8. If $X$ is a limit space as described above, then either CovSpec cut $(X)$ is empty or its universal cover splits isometrically.

Now Theorem 11 in [So], which states that when $X$ doesn't have the loops to infinity property then $X$ has a split double cover and $X$ has a compact soul, was never extended. Its proof involves differentiation. In light of Cheeger's recent work on differentiability of metric measure spaces, we conjecture that the full theorem holds. 
Conjecture 6.9. If $X$ is a limit space as in the above paragraph, then either $X$ has the loops to infinity property or it has a split double cover and it is the flat normal bundle over a compact totally geodesic soul.

If Conjecture 6.9 holds, then the proof of Theorem 6.6 extends to that setting and the following conjecture holds:

Conjecture 6.10. Theorem 6.6 holds for such limit spaces $X$.

Conjecture 6.10 is of particular interest because it has implications to manifolds with $R i c c i \geq-(n-1)$. In particular, the application looks something like a Margulis lemma but with distinct implications:

Conjecture 6.11. Given a complete Riemannian $M^{n}$ with Ricci $\geq-1$ or a Gromov-Hausdorff limit of such spaces, for all $b>a>0$ there exists $\rho=\rho(a, b, n)$ such that

$$
\operatorname{CovSpec}_{\text {cut }}^{\rho}(M, p) \cap[a \rho, b \rho]=\emptyset
$$

or there is a subscaled soul as in (6.10) near $p$.

Note that $\rho$ does not depend on the manifold or the basepoint. It is essentially saying that the manifold looks locally like a manifold with sect $\geq-1$ in neighborhoods where loops don't slide outward. One might think of this as saying that most small loops on the manifold slide around, and when you hit a location where they don't slide, there is a kind of twisting effect similar to a Moebius strip.

Returning from the intuitive to the concrete, we can prove:

Theorem 6.12. Conjecture 6.10 implies Conjecture 6.11,

Proof of Theorem 6.12. Suppose $\left(M_{i}, d_{i}\right)$ have Ricci $\geq-1$ and $\rho_{i} \rightarrow 0$ with

$$
\delta_{i} \rho_{i} \in \operatorname{CovSpec} c_{c u t}^{\rho_{i}}\left(M_{i}, d_{i}, p_{i}\right),
$$

where $\delta_{i} \in[a, b]$. Rescaling $M_{i}$ by $\rho_{i}$, Lemma 5.23 says

$$
\delta_{i} \in \operatorname{CovSpec}_{\text {cut }}^{1}\left(M_{i}, d_{i} / \rho_{i}, p_{i}\right) .
$$

Gromov's compactness theorem implies that a subsequence of the $\left(M_{i}, p_{i}, d_{i} / \rho_{i}\right)$ converge to some $(Y, y, d)$, which satisfies the conditions of Theorem 6.6 Thus $\operatorname{CovSpec}_{\text {cut }}(Y)=\emptyset$ or a split double cover.

Taking a further subsequence we can guarantee $\delta_{i} \rightarrow \delta \in[a, b]$. By Theorem 5.7 and (5.8),

$$
\delta \in \operatorname{CovSpec}_{\text {cut }}^{1}(Y, d, y) \subset \operatorname{CovSpec}_{\text {cut }}(Y) .
$$

Thus $Y$ has a split double cover and is the flat normal bundle over a compact totally geodesic soul $S$.

Finally we apply Theorem 6.3 to $Y$.

\section{Appendix A}

As the concept of lower semiclosure does not seem to appear in the literature, we include a brief exposition here.

Definition 7.1. A lower semiclosed subset of the real line is a set $A$ such that $\lim _{j \rightarrow \infty} a_{j} \in A$ whenever $a_{j}$ is a decreasing sequence of elements of $A$. 
Definition 7.2. The lower semiclosure of a set $A$, denoted $C l_{\text {lower }}(A)$, is the intersection of all lower semiclosed sets containing $A$.

Lemma 7.3. The lower semiclosure of $A$ is the union of $A$ and the limits of any decreasing sequence of $a_{j} \in A$.

Lemma 7.4. If $x \notin A$ and $A$ is lower semiclosed, then there exists $\epsilon>0$ such that

$$
[x, x+\epsilon) \cap A=\emptyset .
$$

The following theorem implies that $\operatorname{CovSpec}(X)$ and $\operatorname{Cov}_{\operatorname{Spec}}(X)$ are lower semiclosed subsets of $(R)$.

Theorem 7.5. Let $X_{s}$ be a collection of metric spaces parametrized by a real line, $s \in(R)$, such that whenever $s_{1}<s_{2}$ we have that $X_{s_{1}}$ covers $X_{s_{2}}$. Any set $A$ is defined as follows:

$$
A:=\left\{s: \forall s^{\prime}>s X_{s^{\prime}} \neq X_{s}\right\}
$$

then $A$ is lower semiclosed.

Proof. Let $s_{j} \in A$ be a decreasing sequence converging to $s_{\infty}$. We need to show that $s_{\infty} \in A$. Let $s^{\prime}>s_{\infty}$. Then for $j$ sufficiently large, we have $s^{\prime}>s_{j}$. Since $s_{j} \in A$ this means $X_{s^{\prime}} \neq X_{s_{j}}$, so $X_{s_{j}}$ is a nontrivial cover of $X_{s^{\prime}}$. Also, since $X_{s_{\infty}}$ covers $X_{s_{j}}$, it must be a nontrivial cover of $X_{s^{\prime}}$ as well.

Example 7.6. If $A_{j}$ are all lower semiclosed sets, the $\bigcup_{j \in \mathbb{N}} A_{j}$ need not be lower semiclosed. For example, let $A_{j}=\{1+1 / k: k=1,2, \ldots, j\}$.

\section{Appendix B}

This appendix provides a minor correction to SoWei3, adding a hypothesis to Lemma 5.8 [Lemma 8.1 and proving a new related Lemma 8.2 which circumvents the additional hypothesis in Theorem 5.7 of SoWei3, which applied Lemma 5.8. Both Lemmas 8.1 and 8.2 are applied in this paper as well.

In [SoWei3], there is an omission in the statement of Lemma 5.8. The proof requires that the curve be rectifiable as pointed out to us by Conrad Plaut. The corrected statement is:

Lemma 8.1. Given a complete length space $Y$, suppose $C:[0, L] \rightarrow B_{q}(\delta) \subset Y$ is rectifiable. Then $C$ is freely homotopic in $B_{q}(\delta)$ to a product of curves, $C_{i}$, of length $L\left(C_{i}\right)<2 \delta$, all of which lie in $B_{q}(\delta)$.

The proof is as in SoWei3:

Proof of Lemma 8.1. Since $B_{q}(\delta)$ is open and the image of $C$ is closed, there exists $\epsilon>0$ such that $\operatorname{Im}(C) \subset B_{q}(\delta-\epsilon)$. Take a partition, $0=t_{0}<t_{1}<\cdots<t_{k}=L$, such that $t_{j+1}-t_{j}<\epsilon$, and let $\gamma_{j}$ run minimally from $q$ to $C\left(t_{j}\right)$, making sure to choose $\gamma_{0}=\gamma_{k}$. Then $C$ is clearly freely homotopic in $B_{q}(\delta)$ to the combination $\gamma_{j} C\left(\left[t_{j}, t_{j+1}\right]\right) \gamma_{j}^{-1}$, and each of these curves has length $<2(\delta-\epsilon)+\epsilon<2 \delta$.

This lemma was applied to prove Theorem 5.7 of [SoWei3] where the covering spectrum is determined by the marked length spectrum when $X$ is a compact length space. In this appendix we provide a correction of that proof which clarifies why we can select a rectifiable curve before applying the corrected Lemma 5.8. 
It should be noted that the Hawaii Ring with loops of length $1 / n$ has a nonrectifiable curve $C$ which traverses all of its loops by traveling faster and faster. So not every homotopy class of curves in a complete length space contains a rectifiable representative.

Recall that in the definition of the delta covers and $\pi(Y, \delta, p)$, curves $\beta$ are classified according to their location: the fact that they are contained in a ball of radius $\delta$. To prove Theorem 5.7, we showed that we could control the lengths of representatives curves as well using Lemma 8.1. This relationship is correctly stated here, and we provide a proof which clarifies how we can select a rectifiable curve:

Lemma 8.2. Given a complete length space $Y$, suppose $\delta^{\prime}<\delta$ and $\tilde{Y}^{\delta} \neq \tilde{Y}^{\delta^{\prime}}$ or, equivalently, $\pi(Y, \delta, p) \neq \pi\left(Y, \delta^{\prime}, p\right)$. Then there is a rectifiable curve $\beta$ of length $L(\beta)<2 \delta$ and a curve $\alpha$ running from $p$ to $\beta(0)$ such that

$$
\left[\alpha^{-1} \circ \beta \circ \alpha\right] \notin \pi\left(Y, \delta^{\prime}, p\right) .
$$

So $\beta$ lifts closed to $\tilde{X}^{\delta}$ and open to $\tilde{X}^{\delta^{\prime}}$.

Note that by the definitions we already know there exists a $\beta^{\prime}$ satisfying (8.1) whose image lies in a ball of radius $\delta$. Otherwise all the generators of $\pi(Y, \delta, p)$ would already lie in $\pi\left(Y, \delta^{\prime}, p\right)$ and $\tilde{Y}^{\delta}$ would equal $\tilde{Y}^{\delta^{\prime}}$. The difficult part is proving that we can control its length. If we knew such a $\beta^{\prime}$ were rectifiable, then we could apply Lemma 8.1 with $\beta^{\prime}=C$ to get

$$
\left[\alpha^{-1} \circ \beta^{\prime} \circ \alpha\right]=\left[\alpha^{-1} \circ C_{1} \circ \alpha\right] \cdot\left[\alpha^{-1} \circ C_{2} \circ \alpha\right] \cdots\left[\alpha^{-1} \circ C_{N} \circ \alpha\right] .
$$

We could then select one of the $C_{i}$ to be $\beta$, and (8.1) would be satisfied since otherwise our original curve would be in $\pi\left(Y, \delta^{\prime}, p\right)$.

When $\beta^{\prime}$ is not rectifiable, as can occur on the Hawaii Ring, then we need only replace it with a rectifiable curve that has the same lifting properties.

The following proof of Lemma 8.2 begins with a technique suggested by Conrad Plaut to shift a nonrectifiable curve to one with similar lifting properties.

Proof of Lemma 8.2. First we know there is a continuous curve $\beta^{\prime}$ contained in some $B_{q}(\delta)$ which satisfies (8.1). Note that since $[0,1]$ is compact, the image of $\beta^{\prime}$ is a compact set. So there exists an $\epsilon \in\left(0, \delta^{\prime} / 10\right)$ sufficiently small such that for any $t \in S^{1}, B_{\beta^{\prime}(t)}(5 \epsilon) \subset B_{q}(\delta)$. By continuity, we can create a partition $0=t_{0}<t_{1}<t_{2}<\cdots<t_{N}=1$ so that each segment $\beta\left(\left[t_{i}, t_{i+1}\right]\right)$ lies in one of these balls.

Note that a curve, $\eta_{i}$, created by running from $\beta^{\prime}\left(t_{i}\right)$ to $\beta^{\prime}\left(t_{i+1}\right)$ along $\beta^{\prime}\left(\left[t_{i}, t_{i+1}\right]\right)$ and then back to $\beta^{\prime}\left(t_{i}\right)$ along a minimal geodesic, lies in a ball of radius $4 \epsilon<\delta^{\prime}$. Choosing appropriate $\alpha_{i}$ running along $\alpha$ and then up $\beta^{\prime}\left(\left[0, t_{i}\right]\right)$, we see that

$$
\left[\alpha_{i}^{-1} \circ \eta_{i} \circ \alpha_{i}\right] \in \pi\left(Y, \delta^{\prime}, p\right) .
$$

So we can construct a rectifiable curve $\beta$ using piecewise minimizing geodesics running between $\beta^{\prime}\left(t_{i}\right)$. This $\beta$ will lie in $B_{q}(\delta)$, it will be rectifiable, and it will satisfy (8.1), since $\beta^{\prime}$ did but the $\eta_{i}$ did not.

We now apply Lemma 8.1 to this curve $\beta$, and we see it is generated by $\beta_{i}$ of length $\leq 2 \delta$ contained in the same $B_{q}(\delta)$. At least one of these curves must satisfy (8.1), or $\beta$ would not. 
Theorem 5.7 of SoWei3] states that on a compact length space with a universal cover, the marked length spectrum determines the covering spectrum. This proof is now corrected by applying Lemma 8.2 in place of Lemma 5.8 to show that we can use rectifiable representative loops of length $<2 \delta^{\prime}$ for the $\beta_{i}$ near the end of the proof of the theorem. Note that Theorem 2.17 essentially contains a new proof of the same theorem in a simpler language.

\section{REFERENCES}

[Ber] L. Berard-Bergery, Quelques exemples de variétés riemanniennes complètes non compactes à courbure de Ricci positive, C. R. Acad. Sci. Paris Sér. I Math. 302 (1986) 159-161. MR832061 (87g:53058)

[BP1] V.N. Beretovskii, C. Plaut, Uniform Universal Covers of Uniform Spaces, Topology and its Applications, Vol. 154, Issue 8, 2007, pp. 1748-1777. MR.2317077(2008g:55023)

[BP2] V.N. Beretovskii, C. Plaut, Covering R-Trees, arxiv:0707.3609

[BoMe] B.H. Bowditch, G. Mess, A 4-dimensional Kleinian group, Trans. Amer. Math. Soc. 344 (1994), no. 1, 391-405. MR 1240944 (95f:57057)

[BBI] D. Burago, Y. Burago, S. Ivanov, A course in Metric Geometry, Graduate Studies in Mathematics Vol. 33, AMS, 2001.

[Ch] J. Cheeger, Degeneration of Riemannian Metrics under Ricci Curvature Bounds, Lezioni Fermiane [Fermi Lectures], Scuola Normale Superiore, Pisa, 2001, ii+77. MR2006642(2004j:53049)

[ChCo1] J. Cheeger, T. Colding, On the structure of spaces with Ricci curvature bounded below I, J. Diff. Geom. 46 (1997) 406-480. MR.1484888 (98k:53044)

[ChCo2] J. Cheeger, T. Colding, On the structure of spaces with Ricci curvature bounded below III, J. Differential Geom. 54 (2000), no. 1, 37-74. MR.1815411 (2003a:53044)

[ChGr] J. Cheeger, D. Gromoll, On the structure of complete manifolds of nonnegative curvature, Ann. of Math. (2) 96 (1972), 413-443. MR0309010 (46:8121)

[Gr] M. Gromov, Metric structures for Riemannian and non-Riemannian spaces, PM 152, Birkhauser, 1999. MR 1699320 (2000d:53065)

[Ma] W. Massey, A basic course in algebraic topology, GTM 127, Springer-Verlag, 1991. MR.1095046 (92c:55001)

[Me] X. Menguy, Examples with bounded diameter growth and infinite topological type, Duke Math. J. 102 (2000), no. 3, 403-412. MR1756103(2001c:53041)

[Na] P. Nabonnand, Sur les variétés riemanniennes complètes à courbure de Ricci positive, C. R. Acad. Sci. Paris Sér. A-B 291 (1980) A591-A593. MR600003 (81m:53054)

[Pe1] G. Perelman, Proof of the soul conjecture of Cheeger and Gromoll, J. Differential Geom. 40 (1994), no. 1, 209-212. MR.1285534 (95d:53037)

[Pe2] G. Perelman, Alexandrov's spaces with curvatures bounded from below II, preprint.

[Po] L. Potyagaulo, Finitely generated Kleinian groups in 3-space and 3-manifolds of infinite homotopy type, Trans. Amer. Math. Soc. 344 (1994), no. 1, 57-77. MR1250823 (94m:57030)

[Sh] V. A. Sharafutdinov, The Pogorelov-Klingenberg theorem for manifolds homeomorphic to $\mathbb{R}^{n}$, Sib. Math. Zh. 18 (1977) 915-925. MR0487896(58:7488)

[ShSo1] Z. Shen and C. Sormani, The Codimension One Homology of a Complete Manifold with Nonnegative Ricci Curvature, American Journal of Mathematics 123 (2001), no. 3, 515-524. MR.1833151 (2002i:53048)

[ShSo2] Z. Shen and C. Sormani, The Topology of Open Manifolds of Nonnegative Ricci Curvature, Proceedings of the Midwest Geometry Conference, Communications in Mathematical Analysis, Conf. 01 (2008), pp 11-19.

[ShW] Z. Shen, G. Wei, On Riemannian manifolds of almost nonnegative curvature, Indiana Univ. Math. Jour. 40 (1991), 551-565. MR.1119188(92e:53053)

[So] C. Sormani, On Loops Representing Elements of the Fundamental Group of a Complete Manifold with Nonnegative Ricci Curvature, Indiana Journal of Mathematics 50 (2001), no. 4, 1867-1883. MR.1889085 (2003b:53038)

[SoWei1] C. Sormani, G. Wei, Hausdorff Convergence and Universal Covers, Transactions of the American Mathematical Society 353 (2001) 3585-3602. MR 1837249 (2002e:53057) 
[SoWei2] C. Sormani, G. Wei, Universal Covers for Hausdorff Limits of Noncompact Spaces, Transactions of the American Mathematical Society 356 (2004), no. 3, 1233-1270. MR2021619 (2005g:53071)

[SoWei3] C. Sormani and G. Wei, The Covering Spectrum of a Compact Length Space, Journal of Differential Geometry (2004) 35-77. MR2153481 (2006e:58050)

[Sp] E. Spanier, Algebraic Topology, McGraw-Hill, Inc., 1966. MR.0210112 (35:1007)

[Wei] G. Wei, Manifolds with A Lower Ricci Curvature Bound, Surveys in Differential Geometry XI (2007), Metric and Comparison Geometry, eds. J. Cheeger and K. Grove, International Press, Somerville, MA, pp. 203-228.

Graduate School and University Center, CUNy, New York, New York 10016 - And - Lehman College, CUNY, Bronx, New York 10468

E-mail address: sormanic@member.ams.org

Department of Mathematics, University of California, Santa Barbara, Santa BarBARA, CALifornia 93106

E-mail address: wei@math.ucsb.edu 